Supporting Information for

\title{
Discovery of ABBV/GLPG-3221, a Potent Corrector of CFTR for the Treatment of Cystic Fibrosis
}

Marc J. C. Scanio, * Xenia B. Searle, Bo Liu, John R. Koenig, Robert Altenbach, Gregory

Gfesser, Andrew Bogdan, Stephen Greszler, Gang Zhao, Ashvani Singh, Yihong Fan, Andrew

M. Swensen, Timothy Vortherms, Arlene Manelli, Corina Balut, Ying Jia, Wenqing Gao, Hong

Yong, Michael Schrimpf, Chris Tse, Philip Kym and Xueqing Wang

Research and Development, AbbVie Inc., 1 North Waukegan Road, North Chicago, Illinois,

United States

*To whom correspondence should be addressed: Phone: +1-847-935-1064; E-mail: marc.scanio@abbvie.com

The synthesis of compound $\mathbf{1 9}$ is shown in Scheme S1 and the subsequent experimentals.

The synthesis of compounds 5-7 is shown in Scheme S2 and the subsequent experimentals.

The synthesis of compounds $\mathbf{8}$ and $\mathbf{1 0}$ is shown in Scheme S3 and the subsequent experimentals.

The synthesis of compound 9 is shown in Scheme S4 and the subsequent experimentals.

The synthesis of compound $\mathbf{1 1}$ is shown in Scheme S5 and the subsequent experimentals.

The synthesis of compounds $\mathbf{1 2}$ and $\mathbf{1 3}$ is shown in Scheme S6 and the subsequent experimentals.

The synthesis of compound $\mathbf{1 4}$ is shown in Scheme S7 and the subsequent experimentals.

The synthesis of compound $\mathbf{1 5}$ is shown in Scheme S8 and the subsequent experimentals.

The synthesis of compound $\mathbf{1 6}$ is shown in Scheme S9 and the subsequent experimentals.

The synthesis of compound $\mathbf{1 7}$ is shown in Scheme S10 and the subsequent experimentals.

The synthesis of compound $\mathbf{1 8}$ is shown in Scheme S11 and the subsequent experimentals.

Scheme S1. Discovery Synthesis of ABBV/GLPG-3221 (19) 
<smiles>[Z10]OC(=O)CN=Cc1ccccc1C</smiles>

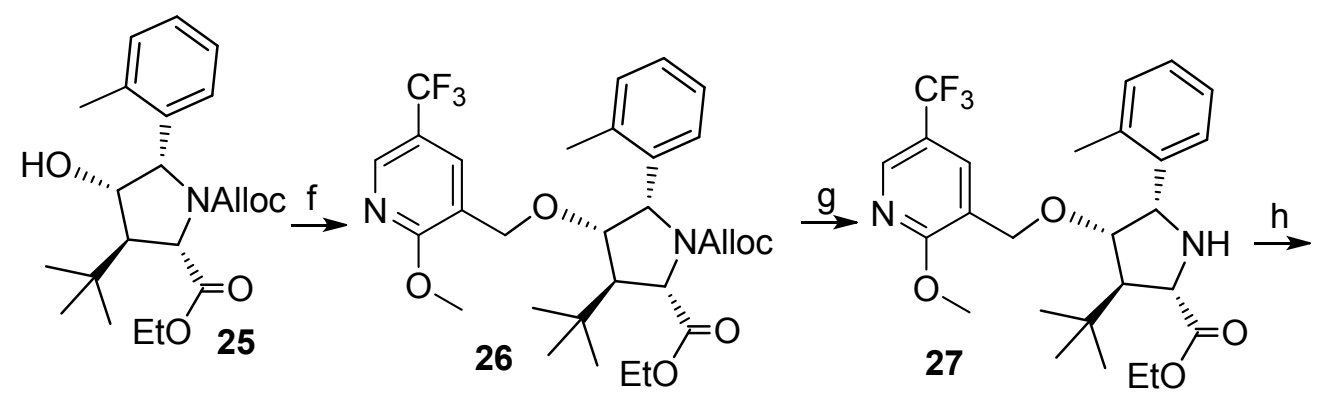<smiles>CCOC(=O)[C@H]1[C@@H](C(C)(C)C)[C@@H](OCc2cc(C(F)(F)F)cnc2OC)[C@H](c2ccccc2C)N1C(=O)[C@H]1CCCCO1</smiles>

\section{ABBV/GLPG-3221}

(19)

aReagents and conditions: (a) $\mathrm{MgSO}_{4}, \quad \mathrm{TEA}, \quad$ 2-methylbenzaldehyde, DCM; $\quad$ (b) (2-(Bis(3,5bis(trifluoromethyl)phenyl)phosphino)-3-((S)-4-isopropyl-4,5-dihydrooxazol-2-yl)cyclopenta-2,4-dien-1-yl)(cyclopenta-2,4-dien1-yl)iron, $\mathrm{Cu}(\mathrm{OTf})_{2}$, KOtBu, (E)-3,3-dimethyl-1-nitrobut-1-ene, THF; (c) allyl chloroformate, toluene; (d) PDC, 6N HCl, Zn dust, (e) $\mathrm{NaBH}_{4}, \mathrm{EtOH}$; (f) KOtBu, 3-(bromomethyl)-2-methoxy-5-(trifluoromethyl)pyridine, DMF; (g) 1,3-Dimethlbarbituric acid, $\mathrm{Pd}\left(\mathrm{PPh}_{3}\right)_{4}$, EtOAc/DCM; (h) Tetrahydropyran-2-carboxylic acid, oxalyl chloride, DMF, DCM; (i) LiOH, MeOH/THF.

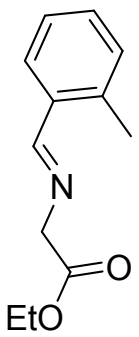

\section{(E)-ethyl 2-((2-methylbenzylidene)amino)acetate (21)}


Ethyl 2-aminoacetate hydrochloride (3.97 g, $28.5 \mathrm{mmol})$ and magnesium sulfate $(3.43 \mathrm{~g}, 28.5$ $\mathrm{mmol})$ were stirred in dichloromethane $(43.1 \mathrm{~mL})$ at ambient temperature, and triethylamine (3.97 mL, $28.5 \mathrm{mmol}$ ) was added. The mixture was stirred for 5 minutes and 2methylbenzaldehyde $(2.97 \mathrm{~mL}, 25.9 \mathrm{mmol})$ was added dropwise. The mixture was stirred at ambient temperature for 16 hours. The solid material was filtered through a disposable plastic frit and washed with dichloromethane. The organic layer was washed with $30 \mathrm{~mL}$ of water, dried over sodium sulfate, filtered, and concentrated to provide (E)-ethyl 2-((2methylbenzylidene)amino)acetate as a thick oil (5.3 g, $100 \%$ yield). ${ }^{1} \mathrm{H}$ NMR $\left(500 \mathrm{MHz}, \mathrm{CDCl}_{3}\right) \delta$ ppm 8.63 (d, J = 1.4 Hz, 1H), 7.96 (dd, J = 7.7, $1.4 \mathrm{~Hz}, 1 \mathrm{H}$ ), 7.35 (td, J = 7.5, 1.5 Hz, 1H), 7.32 $7.25(\mathrm{~m}, 1 \mathrm{H}), 7.25-7.18(\mathrm{~m}, 1 \mathrm{H}), 4.45(\mathrm{~d}, \mathrm{~J}=1.4 \mathrm{~Hz}, 2 \mathrm{H}), 4.28(\mathrm{q}, \mathrm{J}=7.2 \mathrm{~Hz}, 2 \mathrm{H}), 2.55(\mathrm{~s}, 3 \mathrm{H})$, $1.34(\mathrm{t}, \mathrm{J}=7.1 \mathrm{~Hz}, 3 \mathrm{H})$. MS (ESI+) $\mathrm{m} / z 206(\mathrm{M}+\mathrm{H})^{+}$.

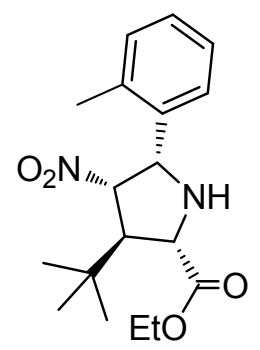

(2S,3R,4S,5S)-ethyl 3-(tert-butyl)-4-nitro-5-(o-tolyl)pyrrolidine-2-carboxylate (22) (2-(Bis(3,5-bis(trifluoromethyl)phenyl)phosphino)-3-((S)-4-isopropyl-4,5-dihydrooxazol-2yl)cyclopenta-2,4-dien-1-yl)(cyclopenta-2,4-dien-1-yl)iron (0.222 g, $0.294 \mathrm{mmol}$ ) and copper (I) triflate dimer, benzene complex $(0.064 \mathrm{~g}, 0.127 \mathrm{mmol})$ were dissolved in tetrahydrofuran (51.7 $\mathrm{mL}$ ) that had been sparged with a stream of nitrogen for 4 hours. The resulting mixture was stirred for 1.5 hours at ambient temperature, and (E)-ethyl 2-((2methylbenzylidene)amino)acetate $(5.31 \mathrm{~g}, 25.9 \mathrm{mmol})$ was added after cooling to $<5{ }^{\circ} \mathrm{C}$ in an ice-water bath. Potassium 2-methylpropan-2-olate $(0.203 \mathrm{~mL}, 0.203 \mathrm{mmol})$ was added dropwise, followed by addition of (E)-3,3-dimethyl-1-nitrobut-1-ene (3.51 g, $27.2 \mathrm{mmol})$ neat over 25 minutes, maintaining an internal temperature $<10^{\circ} \mathrm{C}$. After the addition was complete, the reaction was stirred for 90 minutes at the same temperature. The reaction mixture was diluted with methyl tert-butyl ether $(100 \mathrm{~mL})$ and stirred with $50 \mathrm{~mL}$ of saturated aqueous ammonium chloride at ambient temperature for 15 minutes. The organic layer was washed with saturated sodium bicarbonate and brine, dried over sodium sulfate, and filtered. The filtrate was 
concentrated and was diluted with $80 \mathrm{~mL}$ of heptanes and the solvent was reduced in volume until a solid precipitated out. The mixture was cooled in an ice bath to $<5^{\circ} \mathrm{C}$ for 15 minutes, and the resulting material was filtered, washed with $20 \mathrm{~mL}$ of heptanes, and dried to constant weight in a vacuum oven to provide ( $2 S, 3 R, 4 S, 5 S)$-ethyl 3-(tert-butyl)-4-nitro-5-(o-tolyl)pyrrolidine-2carboxylate (4.85 g, $14.50 \mathrm{mmol}, 56 \%$ yield). ${ }^{1} \mathrm{H}$ NMR (501 MHz, $\left.\mathrm{CDCl}_{3}\right) \delta \mathrm{ppm} 7.34-7.27$ (m, 1H), 7.27 - $7.16(\mathrm{~m}, 3 \mathrm{H}), 5.18(\mathrm{dd}, \mathrm{J}=6.1,2.6 \mathrm{~Hz}, 1 \mathrm{H}), 4.55(\mathrm{dd}, \mathrm{J}=10.1,5.9 \mathrm{~Hz}, 1 \mathrm{H}), 4.35$ $(\mathrm{qd}, \mathrm{J}=7.2,1.2 \mathrm{~Hz}, 2 \mathrm{H}), 3.81(\mathrm{t}, \mathrm{J}=7.1 \mathrm{~Hz}, 1 \mathrm{H}), 3.31(\mathrm{~s}, 1 \mathrm{H}), 3.07(\mathrm{dd}, \mathrm{J}=7.3,2.6 \mathrm{~Hz}, 1 \mathrm{H})$, $2.41(\mathrm{~s}, 3 \mathrm{H}), 1.38(\mathrm{t}, \mathrm{J}=7.1 \mathrm{~Hz}, 3 \mathrm{H}), 1.08(\mathrm{~s}, 9 \mathrm{H})$. MS (APCI+) $\mathrm{m} / z 335(\mathrm{M}+\mathrm{H})^{+}$

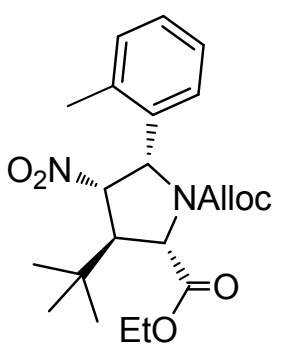

(2S,3R,4S,5S)-1-allyl 2-ethyl 3-(tert-butyl)-4-nitro-5-(o-tolyl)pyrrolidine-1,2-dicarboxylate (23) To a mixture of Compound 22 (946 mg, $2.83 \mathrm{mmol})$ in toluene $(3 \mathrm{~mL})$ and saturated aqueous $\mathrm{NaHCO}_{3}(3 \mathrm{~mL})$ was added allyl carbonochloridate $(360 \mu \mathrm{L}, 3.39 \mathrm{mmol})$ dropwise at room temperature. The mixture was stirred at room temperature overnight. The reaction was poured into water $(50 \mathrm{~mL})$ and extracted with dichloromethane $(50 \mathrm{~mL})$. The organic layer was washed with brine and concentrated in vacuo. Purification by silica gel chromatography, eluting with ethyl acetate in heptane, provided the title compound, $1.22 \mathrm{~g}$ (quantitative yield). ${ }^{1} \mathrm{H}$ NMR (400 MHz, DMSO- $d_{6}$ ) $\delta$ ppm $7.79(\mathrm{dd}, \mathrm{J}=7.8,1.9 \mathrm{~Hz}, 1 \mathrm{H}), 7.24$ - $7.04(\mathrm{~m}, 3 \mathrm{H}), 5.69$ (ddt, J = 17.4, 10.5, 5.1 Hz, 1H), $5.60(\mathrm{~d}, \mathrm{~J}=8.9 \mathrm{~Hz}, 1 \mathrm{H}), 5.55(\mathrm{dd}, \mathrm{J}=8.9,3.1 \mathrm{~Hz}, 1 \mathrm{H}), 5.07$ - $4.96(\mathrm{~m}, 2 \mathrm{H})$, $4.56(\mathrm{~d}, \mathrm{~J}=3.9 \mathrm{~Hz}, 1 \mathrm{H}), 4.42(\mathrm{dtd}, \mathrm{J}=5.2,1.7,0.7 \mathrm{~Hz}, 2 \mathrm{H}), 4.26(\mathrm{q}, \mathrm{J}=7.1 \mathrm{~Hz}, 2 \mathrm{H}), 3.08(\mathrm{t}, \mathrm{J}=$

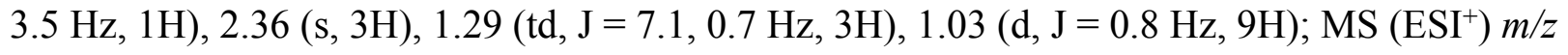
$419.0(\mathrm{M}+\mathrm{H})^{+}$. 


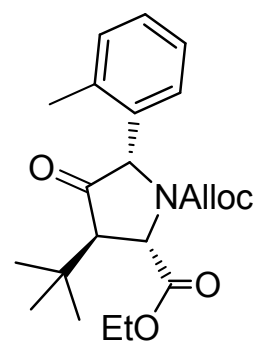

(2S,3R,5S)-1-allyl 2-ethyl 3-(tert-butyl)-4-oxo-5-(o-tolyl)pyrrolidine-1,2-dicarboxylate (24)

Pyridinium dichromate $(2.74 \mathrm{~g}, 7.29 \mathrm{mmol})$ was dissolved in $6 \mathrm{~N}$ aqueous $\mathrm{HCl}(64.0 \mathrm{~mL})$ and cooled in an ice bath. Zinc dust $(5.24 \mathrm{~g}, 80 \mathrm{mmol})$ was added in portions, then the flask was removed from the ice bath, and the reaction mixture was stirred at room temperature for 75 minutes (until zinc had dissolved and bubbling had stopped). The blue solution was transferred via cannula over about 20 minutes to a refluxing solution of Compound 23 (1.22 g, $2.92 \mathrm{mmol}$ ) in ethanol $(32 \mathrm{~mL})$. The reaction mixture was refluxed overnight. The reaction mixture was cooled to room temperature, and extracted three times with dichloromethane. The combined organic extracts were dried over $\mathrm{Na}_{2} \mathrm{SO}_{4}$, filtered, and concentrated in vacuo. The resulting material was taken up in $5.5 \mathrm{~mL}$ of ethanol and treated with a solution (prepared at $0^{\circ} \mathrm{C}$ ) of 1.85 $\mathrm{mL}$ acetyl chloride in $9 \mathrm{~mL}$ ethanol. The reaction mixture was refluxed for 1 hour. After this time, the mixture was concentrated in vacuo, and the residue was purified by silica gel chromatography, eluting with 0 to $40 \%$ ethyl acetate-heptanes to provide the title compound, $0.693 \mathrm{~g},\left(61 \%\right.$ yield). ${ }^{1} \mathrm{H}$ NMR (400 MHz, DMSO- $\left.d_{6}\right) \delta \mathrm{ppm} 7.68$ - 7.61 (m, 1H), $7.21-7.06$ (m, 3H), $5.70(\mathrm{~m}, 1 \mathrm{H}), 5.23(\mathrm{~s}, 1 \mathrm{H}), 5.08-4.97(\mathrm{~m}, 2 \mathrm{H}), 4.63(\mathrm{~d}, \mathrm{~J}=5.1 \mathrm{~Hz}, 1 \mathrm{H}), 4.46(\mathrm{~m}, 2 \mathrm{H})$, $4.28-4.18(\mathrm{~m}, 2 \mathrm{H}), 2.64(\mathrm{dd}, \mathrm{J}=5.3,1.0 \mathrm{~Hz}, 1 \mathrm{H}), 2.38(\mathrm{~s}, 3 \mathrm{H}), 1.26(\mathrm{t}, \mathrm{J}=7.1 \mathrm{~Hz}, 3 \mathrm{H}), 1.06(\mathrm{~s}$, 9H); $\mathrm{MS}\left(\mathrm{ESI}^{+}\right) m / z 388.0(\mathrm{M}+\mathrm{H})^{+}$.

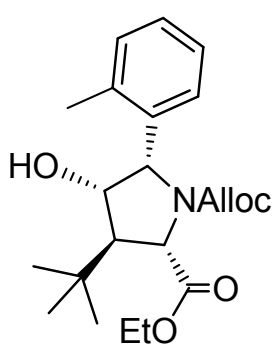

(2S,3R,4S,5S)-1-allyl 2-ethyl 3-(tert-butyl)-4-hydroxy-5-(o-tolyl)pyrrolidine-1,2-dicarboxylate (25) A solution of Compound $24(0.693 \mathrm{~g}, 1.789 \mathrm{mmol})$ in ethanol $(8.9 \mathrm{~mL})$ was cooled to $-10^{\circ}$ and then treated with one portion of sodium borohydride $(0.081 \mathrm{~g}, 2.146 \mathrm{mmol})$. The reaction stirred 
at $-10{ }^{\circ} \mathrm{C}$ to $-5{ }^{\circ} \mathrm{C}$ for 90 minutes, and it was quenched carefully with $0.6 \mathrm{~mL}$ acetone. The mixture was concentrated in vacuo, and the resulting material was taken up in $10 \mathrm{~mL}$ ethyl acetate and stirred vigorously with $10 \mathrm{~mL}$ of saturated aqueous $\mathrm{NaHCO}_{3}$ solution for 30 minutes. The mixture was transferred to a separatory funnel, the phases were separated, and the aqueous layer was extracted twice more with ethyl acetate $(10 \mathrm{~mL}$ each). The combined organics were dried over $\mathrm{Na}_{2} \mathrm{SO}_{4}$, filtered, and concentrated in vacuo, and the crude product was purified by silica gel chromatography, eluting with 5 to $40 \%$ ethyl acetate-heptanes to provide the title compound, $0.280 \mathrm{~g}$ (40\% yield). ${ }^{1} \mathrm{H}$ NMR (400 MHz, DMSO- $\left.d_{6}\right) \delta \mathrm{ppm} 7.85(\mathrm{~m}, 1 \mathrm{H}), 7.11-$ $7.01(\mathrm{~m}, 3 \mathrm{H}), 5.73-5.61(\mathrm{~m}, 1 \mathrm{H}), 5.08$ (d, J = 6.6 Hz, 1H), $4.99(\mathrm{~m}, 2 \mathrm{H}), 4.45$ - $4.33(\mathrm{~m}, 3 \mathrm{H})$, $4.30(\mathrm{~m}, 1 \mathrm{H}), 4.22$ - $4.12(\mathrm{~m}, 2 \mathrm{H}), 3.82(\mathrm{~m}, 1 \mathrm{H}), 2.29(\mathrm{~s}, 3 \mathrm{H}), 2.28(\mathrm{~m}, 1 \mathrm{H}), 1.24(\mathrm{t}, \mathrm{J}=7.1 \mathrm{~Hz}$, 3H), 0.99 (s, 9H); MS (ESI $\left.{ }^{+}\right) m / z 390.1(\mathrm{M}+\mathrm{H})$.

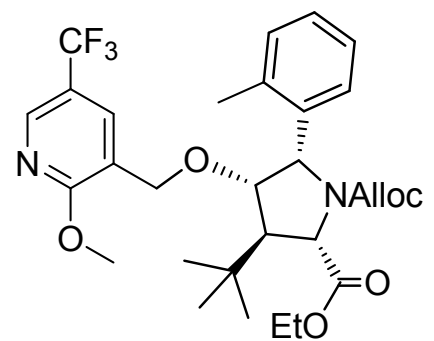

(2S,3R,4S,5S)-1-allyl 2-ethyl 3-(tert-butyl)-4-((2-methoxy-5-(trifluoromethyl)pyridin-3-yl)methoxy)5-(o-tolyl)pyrrolidine-1,2-dicarboxylate (26)

Compound 25 (0.280 g, $0.719 \mathrm{mmol})$, dried azeotropically with toluene, was dissolved in dimethylformamide $(7.2 \mathrm{~mL})$, and the solution was cooled to $0{ }^{\circ} \mathrm{C}$. Potassium 2-methylpropan-2-olate solution (1M in tetrahydrofuran) $(0.79 \mathrm{~mL}, 0.79 \mathrm{mmol})$ was added dropwise, followed by dropwise addition of 3-(bromomethyl)-2-methoxy-5-(trifluoromethyl)pyridine (0.233 g, $0.863 \mathrm{mmol})$. The reaction mixture stirred at $0{ }^{\circ} \mathrm{C}$ for 45 minutes, the mixture was poured into $30 \mathrm{~mL}$ of saturated aqueous $\mathrm{NH}_{4} \mathrm{Cl}$ solution and extracted three times with methyl tert-butyl ether $(3 \times 30 \mathrm{~mL})$. The combined organics were dried over $\mathrm{Na}_{2} \mathrm{SO}_{4}$, filtered and concentrated in vacuo, and the crude product was purified by silica gel chromatography, eluting with 0 to $30 \%$ methyl tert-butyl ether-heptanes to provide the title compound, $0.224 \mathrm{~g}\left(54 \%\right.$ yield). ${ }^{1} \mathrm{H}$ NMR (400 MHz, DMSO- $\left.d_{6}\right) \delta \mathrm{ppm} 8.28(\mathrm{~m}, 1 \mathrm{H}), 7.85(\mathrm{tt}, \mathrm{J}=4.6,3.0 \mathrm{~Hz}, 0 \mathrm{H})$, 7.18 - $7.13(\mathrm{~m}, 1 \mathrm{H}), 7.04-6.96(\mathrm{~m}, 3 \mathrm{H}), 5.69$ (ddt, $\mathrm{J}=16.3,10.5,5.1 \mathrm{~Hz}, 1 \mathrm{H}), 5.19(\mathrm{~d}, \mathrm{~J}=5.8 \mathrm{~Hz}, 1 \mathrm{H})$, 5.06 - $4.95(\mathrm{~m}, 2 \mathrm{H}), 4.48(\mathrm{~d}, \mathrm{~J}=2.3 \mathrm{~Hz}, 1 \mathrm{H}), 4.40(\mathrm{dq}, \mathrm{J}=5.1,1.5 \mathrm{~Hz}, 2 \mathrm{H}), 4.34-4.22(\mathrm{~m}, 2 \mathrm{H}), 4.11$ (q, $\mathrm{J}=7.0 \mathrm{~Hz}, 2 \mathrm{H}), 3.86(\mathrm{~s}, 3 \mathrm{H}), 3.83(\mathrm{dt}, \mathrm{J}=13.7,1.1 \mathrm{~Hz}, 1 \mathrm{H}), 2.86-2.79(\mathrm{~m}, 1 \mathrm{H}), 2.51(\mathrm{~m}, 1 \mathrm{H}), 2.31(\mathrm{~s}$, $3 \mathrm{H}), 1.15(\mathrm{t}, \mathrm{J}=7.1 \mathrm{~Hz}, 3 \mathrm{H}), 1.03(\mathrm{~s}, 9 \mathrm{H})$; $\mathrm{MS}\left(\mathrm{ESI}^{+}\right) \mathrm{m} / z 578.9(\mathrm{M}+\mathrm{H})^{+}$. 


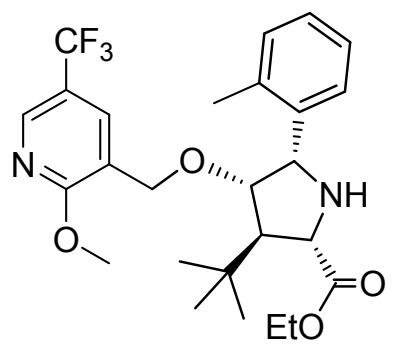

(2S,3R,4S,5S)-ethyl 3-(tert-butyl)-4-((2-methoxy-5-(trifluoromethyl)pyridin-3-yl)methoxy)-5-(otolyl)pyrrolidine-2-carboxylate (27)

Compound 26 (0.224 g, $0.387 \mathrm{mmol})$ was dissolved in ethyl acetate $(2 \mathrm{~mL})$ and dichloromethane $(2 \mathrm{~mL})$, and the solution was treated with 1,3-dimethylpyrimidine-2,4,6(1H,3H,5H)-trione $(0.121 \mathrm{~g}, 0.774 \mathrm{mmol})$ and tetrakis(triphenylphosphine)palladium $(4.5 \mathrm{mg}, 3.87 \mu \mathrm{mol})$. The reaction stirred at room temperature overnight. After this time, the reaction mixture was diluted with methyl tert-butyl ether $(20 \mathrm{~mL})$ and stirred with $20 \mathrm{~mL}$ of $10 \%$ aqueous $\mathrm{Na}_{2} \mathrm{CO}_{3}$ solution for 15 minutes. The phases were then separated, and the organic layer was washed with brine, dried over $\mathrm{Na}_{2} \mathrm{SO}_{4}$, filtered, and concentrated in vacuo. Silica gel chromatography, eluting with 0 to $30 \%$ methyl tert-butyl ether-heptanes, provided the title compound, $0.176 \mathrm{~g}\left(92 \%\right.$ yield). ${ }^{1} \mathrm{H}$ NMR (400 MHz, DMSO- $\left.d_{6}\right) \delta \mathrm{ppm} 8.30(\mathrm{~s}, 1 \mathrm{H}), 7.66-7.50(\mathrm{~m}, 1 \mathrm{H}), 7.35(\mathrm{~d}, \mathrm{~J}$ $=2.4 \mathrm{~Hz}, 1 \mathrm{H}), 7.05(\mathrm{~m}, 3 \mathrm{H}), 4.34-4.10(\mathrm{~m}, 5 \mathrm{H}), 4.10-3.99(\mathrm{~m}, 1 \mathrm{H}), 3.86(\mathrm{~s}, 3 \mathrm{H}), 3.79(\mathrm{~d}, \mathrm{~J}=13.9 \mathrm{~Hz}$, $1 \mathrm{H}), 3.63(\mathrm{~s}, 1 \mathrm{H}), 2.38(\mathrm{dd}, \mathrm{J}=6.5,1.7 \mathrm{~Hz}, 1 \mathrm{H}), 2.32(\mathrm{~s}, 3 \mathrm{H}), 1.21(\mathrm{t}, \mathrm{J}=7.0 \mathrm{~Hz}, 3 \mathrm{H}), 0.99(\mathrm{~s}, 9 \mathrm{H})$; MS $\left(\mathrm{APCI}^{+}\right) \mathrm{m} / z$ 495.6 (M+H) ${ }^{+}$.<smiles>CCOC(=O)[C@H]1[C@@H](C(C)(C)C)[C@@H](OCc2cc(C(F)(F)F)cnc2OC)[C@H](c2ccccc2C)N1C(=O)[C@H]1CCCCO1</smiles>

Ethyl (2S,3R,4S,5S)-3-(tert-butyl)-4-((2-methoxy-5-(trifluoromethyl)pyridin-3-yl)methoxy)-1-((S)tetrahydro-2H-pyran-2-carbonyl)-5-(o-tolyl)pyrrolidine-2-carboxylate (28)

Tetrahydropyran-2-carboxylic acid $(64.1 \mathrm{mg}, 0.493 \mathrm{mmol})$ was dissolved in dichloromethane $(2 \mathrm{~mL})$. Oxalyl chloride ( $2 \mathrm{M}$ in dichloromethane, $300 \mu \mathrm{L}, 0.60 \mathrm{mmol}$ ) was added followed by dimethylformamide $(25 \mu \mathrm{L})$. The reaction was stirred at ambient temperature for 3 hours, at which point it was concentrated, redissolved in dichloromethane $(1 \mathrm{~mL})$, and concentrated again. The residue was taken up in dichloromethane $(3 \times 1 \mathrm{~mL})$ and added to a solution of Compound $27(116 \mathrm{mg}, 0.235 \mathrm{mmol})$ and triethylamine $(130 \mu \mathrm{L}, 0.933 \mathrm{mmol})$ in dichloromethane $(3 \mathrm{~mL})$. The reaction was stirred at ambient 
temperature for 17 hours. After this time, the mixture was diluted with dichloromethane $(100 \mathrm{~mL})$ and washed twice with saturated $\mathrm{NaHCO}_{3}$, and once with brine. The organic layer was dried over $\mathrm{Na}_{2} \mathrm{SO}_{4}$, filtered, and concentrated. The residue was purified by reverse-phase preparative HPLC on a Phenomenex ${ }^{\circledR}$ Luna ${ }^{\circledR}$ C8(2) $5 \mu \mathrm{m} 100 \AA$ AXIA $^{\mathrm{TM}}$ column $(30 \mathrm{~mm} \times 75 \mathrm{~mm})$. A gradient of acetonitrile (A) and $0.1 \%$ trifluoroacetic acid in water (B) was used, at a flow rate of $50 \mathrm{~mL} /$ minute $(0-0.5$ minutes $10 \%$ A, 0.5-7.0 minutes linear gradient 10-95\% A, 7.0-10.0 minutes 95\% A, 10.0-12.0 minutes linear gradient $95-10 \% \mathrm{~A})$. The title compound was the second eluting diastereomer and was isolated as the TFA salt (43.1 mg, 26\%). ${ }^{1} \mathrm{H}$ NMR (400 MHz, DMSO- $\left.d_{6}, 120^{\circ} \mathrm{C}\right) \delta \mathrm{ppm} 8.28(\mathrm{~s}, 1 \mathrm{H}), 8.01-7.91(\mathrm{~m}$, 1H), $7.20(\mathrm{~d}, J=2.4 \mathrm{~Hz}, 1 \mathrm{H}), 7.13-6.91(\mathrm{~m}, 3 \mathrm{H}), 5.56(\mathrm{~s}, 1 \mathrm{H}), 4.68(\mathrm{~s}, 1 \mathrm{H}), 4.33-4.21(\mathrm{~m}, 3 \mathrm{H}), 4.09$ (qd, $J=7.1,2.8 \mathrm{~Hz}, 2 \mathrm{H}), 3.86(\mathrm{~s}, 3 \mathrm{H}), 3.82(\mathrm{~d}, J=13.9 \mathrm{~Hz}, 1 \mathrm{H}), 3.75(\mathrm{~d}, J=11.4 \mathrm{~Hz}, 1 \mathrm{H}), 2.90(\mathrm{~s}, 1 \mathrm{H})$, $2.42(\mathrm{t}, J=1.3 \mathrm{~Hz}, 1 \mathrm{H}), 2.34(\mathrm{~s}, 3 \mathrm{H}), 1.77-1.22(\mathrm{~m}, 6 \mathrm{H}), 1.14$ (t, $J=7.0 \mathrm{~Hz}, 3 \mathrm{H}), 1.02(\mathrm{~s}, 9 \mathrm{H})$; MS $(\mathrm{ESI}+) \mathrm{m} / z 607(\mathrm{M}+\mathrm{H})^{+}$.

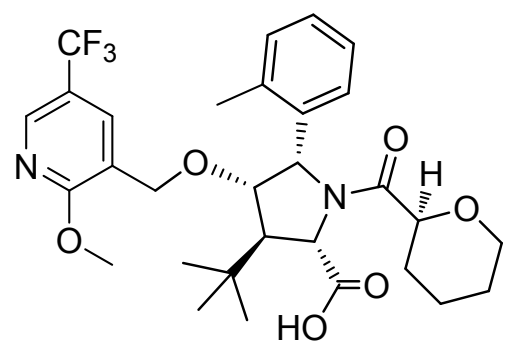

(2S,3R,4S,5S)-3-tert-Butyl-4-\{[2-methoxy-5-(trifluoromethyl)pyridin-3-yl]methoxy\}-5-(2methylphenyl)-1-[(2S)-oxane-2-carbonyl]pyrrolidine-2-carboxylic acid (19, ABBV-3221) Compound 28 (40.7 mg, $0.056 \mathrm{mmol})$ was dissolved in methanol $(1 \mathrm{~mL})$, and tetrahydrofuran $(1 \mathrm{~mL})$. Aqueous $\mathrm{LiOH}(1 \mathrm{M}, 0.5 \mathrm{~mL}, 0.5 \mathrm{mmol})$ was added and the reaction was heated to $50^{\circ} \mathrm{C}$ for 16 hours. The reaction was quenched by the addition of $1 \mathrm{M}$ aqueous $\mathrm{HCl}(0.5 \mathrm{~mL})$, concentrated and purified by reverse-phase preparative HPLC on a Phenomenex ${ }^{\circledR}$ Luna ${ }^{\circledR}$ C8(2) $5 \mu \mathrm{m} 100 \AA ̊$ AXIA ${ }^{\mathrm{TM}}$ column $(30 \mathrm{~mm}$ $\times 75 \mathrm{~mm}$ ). A gradient of acetonitrile (A) and $0.1 \%$ trifluoroacetic acid in water (B) was used, at a flow rate of $50 \mathrm{~mL} /$ minute $(0-0.5$ minutes $10 \% \mathrm{~A}, 0.5-7.0$ minutes linear gradient $10-95 \% \mathrm{~A}, 7.0-10.0$ minutes 95\% A, 10.0-12.0 minutes linear gradient $95-10 \%$ A) to provide the title compound $(27.0 \mathrm{mg}, 69 \%)$ as the TFA salt. ${ }^{1} \mathrm{H}$ NMR $\left(400 \mathrm{MHz}, \mathrm{DMSO}-d_{6}, 120^{\circ} \mathrm{C}\right) \delta \mathrm{ppm} 8.31-8.24(\mathrm{~m}, 1 \mathrm{H}), 8.08-7.99(\mathrm{~m}, 1 \mathrm{H}), 7.18$ $(\mathrm{d}, \mathrm{J}=2.4 \mathrm{~Hz}, 1 \mathrm{H}), 7.11$ - $6.98(\mathrm{~m}, 3 \mathrm{H}), 5.56(\mathrm{~s}, 1 \mathrm{H}), 4.64(\mathrm{~s}, 1 \mathrm{H}), 4.32$ - $4.24(\mathrm{~m}, 2 \mathrm{H}), 3.91$ - 3.82 (m, $5 \mathrm{H}), 3.76(\mathrm{~d}, \mathrm{~J}=11.3 \mathrm{~Hz}, 2 \mathrm{H}), 2.50(\mathrm{~s}, 1 \mathrm{H}), 2.33(\mathrm{~s}, 3 \mathrm{H}), 1.74$ - $1.23(\mathrm{~m}, 6 \mathrm{H}), 1.02(\mathrm{~s}, 9 \mathrm{H})$; MS (ESI+) $m / z 579.3(\mathrm{M}+\mathrm{H})^{+}$.

\section{Scheme S2. Discovery Synthesis of Compounds 5-7}



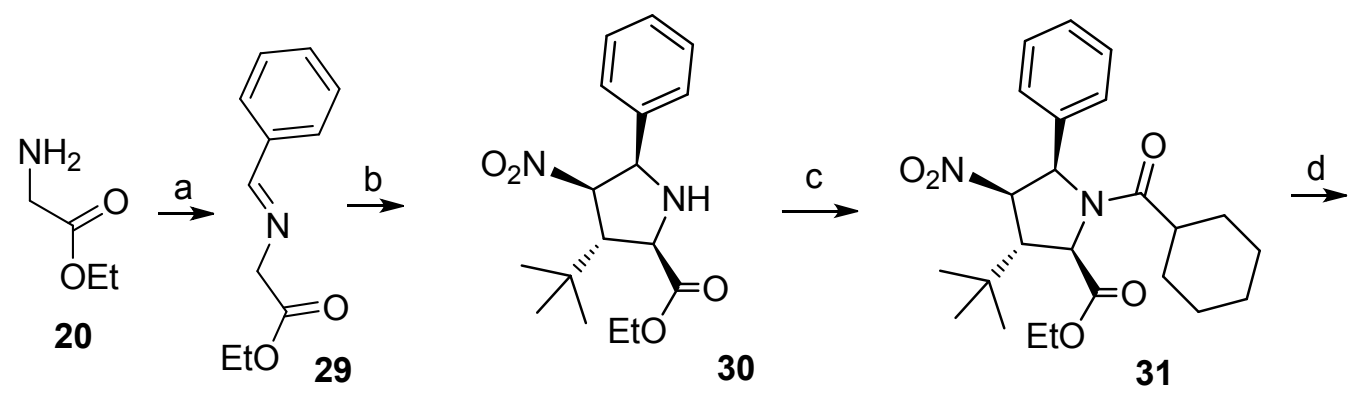<smiles>CCOC(=O)[C@H]1[C@@H](C(C)(C)C)C(=O)[C@@H](c2ccccc2)N1C(=O)C1CCCCC1</smiles>

32<smiles>CCOC(=O)[C@H]1[C@@H](C(C)(C)C)[C@@H](O)[C@H](c2ccccc2)N1C(=O)C1CCCCC1</smiles>

33<smiles>COc1ccc(C(F)(F)F)cc1CO[C@@H]1[C@H](c2ccccc2)N(C(=O)C2CCCCC2)[C@H](C(=O)O)[C@@H]1C(C)(C)C</smiles>

5<smiles>COc1ccc(C(F)(F)F)cc1CO[C@@H]1[C@H](c2ccccc2)N(C(=O)C2CCCCC2)[C@H](C(=O)O)[C@@H]1C(C)(C)C</smiles><smiles>COc1ccc(C(F)(F)F)cc1CO[C@@H]1[C@H](c2ccccc2)N(C(=O)C2CCCCC2)[C@H](C(=O)O)[C@@H]1C(C)(C)C</smiles>

aReagents and conditions: (a) $\mathrm{MgSO}_{4}$, TEA, 2-methylbenzaldehyde, DCM; (b) $\mathrm{Ag}(\mathrm{OAc})_{2},(E)$-3,3-dimethyl-1-nitrobut-1-ene, TEA, $3 \AA$ molecular sieves, toluene; (c) cyclohexanecarbonyl chloride, TEA, DCM; (d) PDC, 6N HCl, Zn dust, (e) $\mathrm{NaBH}_{4}$, $\mathrm{MeOH}$; (f) NaH, 2-(bromomethyl)-1-methoxy-4-(trifluoromethyl)benzene, DMF; LiOH, MeOH; (g) chiral preparative SFC.

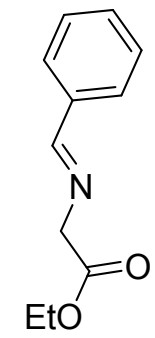

\section{(E)-ethyl 2-(benzylideneamino)acetate (29)}


To a mixture of glycine ethyl ester hydrochloride $(7.23 \mathrm{~g}, 51.8 \mathrm{mmol})$ and magnesium sulfate $(7.09 \mathrm{~g}$, $58.9 \mathrm{mmol})$ in dichloromethane $(80 \mathrm{~mL})$ was added triethylamine $(7.22 \mathrm{~mL}, 51.8 \mathrm{mmol})$. The mixture was stirred at ambient temperature for 20 minutes, and benzaldehyde $(4.79 \mathrm{~mL}, 47.1 \mathrm{mmol})$ was added dropwise. The mixture was stirred overnight. The reaction mixture was filtered and the solid was washed with dichloromethane $(20 \mathrm{~mL}$ x 2). The combined organic layers were washed with brine, dried over $\mathrm{MgSO}_{4}$, filtered and concentrated to yield (E)-ethyl 2-(benzylideneamino)acetate $8.2 \mathrm{~g},\left(91 \%\right.$ yield). ${ }^{1} \mathrm{H}$ NMR (400 MHz, $\left.\mathrm{CDCl}_{3}\right) \delta \mathrm{ppm} 8.30(\mathrm{~s}, 1 \mathrm{H}), 7.83$ - $7.71(\mathrm{~m}, 2 \mathrm{H}), 7.48$ - $7.37(\mathrm{~m}, 2 \mathrm{H}), 4.40(\mathrm{~d}, \mathrm{~J}=1.4$ $\mathrm{Hz}, 2 \mathrm{H}), 4.24(\mathrm{q}, \mathrm{J}=7.1 \mathrm{~Hz}, 2 \mathrm{H}), 1.30(\mathrm{t}, \mathrm{J}=7.1 \mathrm{~Hz}, 3 \mathrm{H})$; MS (ESI+) $m / z 292.1(\mathrm{M}+\mathrm{H})^{+}$.

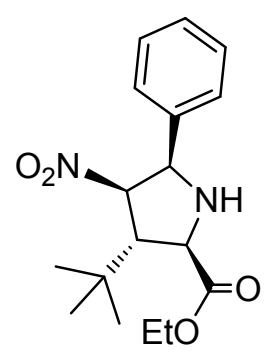

rac-(2R,3R,4R,5R)-ethyl 3-(tert-butyl)-4-nitro-5-phenylpyrrolidine-2-carboxylate (30)

To a solution of Compound $29(1.0 \mathrm{~g}, 5.23 \mathrm{mmol})$ and $(E)-3,3$-dimethyl-1-nitrobut-1-ene $(0.810 \mathrm{~g}, 6.28$ $\mathrm{mmol})$ in toluene $(30 \mathrm{~mL})$ cooled in an ice-bath was added silver acetate $(1.309 \mathrm{~g}, 7.84 \mathrm{mmol})$ and $3 \AA$ molecular sieves. Triethylamine $(1.458 \mathrm{~mL}, 10.46 \mathrm{mmol})$ was added slowly to the well stirred reaction mixture. After stirring at $0{ }^{\circ} \mathrm{C}$ for 10 minutes, the reaction mixture was allowed to warm to ambient temperature and was stirred for another 4 hours. Saturated aqueous ammonium chloride was added, the precipitate was filtered off and the residue was extracted with ether. The combined organic fractions were dried over $\mathrm{MgSO}_{4}$, filtered, concentrated, and purified by chromatography on $40 \mathrm{~g}$ silica gel cartridge, eluting with ethyl acetate in heptane, 0-40\% gradient to provide the title compound (1.6 g, $95 \%$ yield). ${ }^{1} \mathrm{H}$ NMR (400 MHz, $\left.\mathrm{CDCl}_{3}\right) \delta \mathrm{ppm} 7.39$ - $7.26(\mathrm{~m}, 5 \mathrm{H}), 5.12(\mathrm{dd}, \mathrm{J}=6.0,2.5 \mathrm{~Hz}, 1 \mathrm{H}), 4.44(\mathrm{~d}, \mathrm{~J}$ $=5.7 \mathrm{~Hz}, 1 \mathrm{H}), 4.31(\mathrm{q}, \mathrm{J}=7.2 \mathrm{~Hz}, 2 \mathrm{H}), 3.81(\mathrm{~d}, \mathrm{~J}=7.1 \mathrm{~Hz}, 1 \mathrm{H}), 3.30(\mathrm{~s}, 1 \mathrm{H}), 2.95(\mathrm{dd}, \mathrm{J}=7.1,2.5 \mathrm{~Hz}$, 1H), $1.34(\mathrm{t}, \mathrm{J}=7.2 \mathrm{~Hz}, 3 \mathrm{H}), 1.06(\mathrm{~s}, 9 \mathrm{H}) ; \mathrm{MS}(\mathrm{ESI}+) \mathrm{m} / \mathrm{z} 321(\mathrm{M}+\mathrm{H})^{+}$.

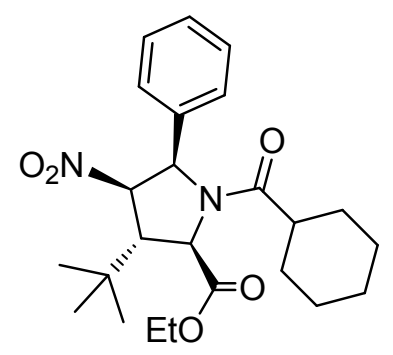


rac-(2R,3S,4R,5R)-ethyl 3-(tert-butyl)-1-(cyclohexanecarbonyl)-4-nitro-5-phenylpyrrolidine-2carboxylate (31)

A solution of Compound $30(5.0 \mathrm{~g}, 15.61 \mathrm{mmol})$ and triethylamine $(3.27 \mathrm{~mL}, 23.41 \mathrm{mmol})$ in dichloromethane $(40 \mathrm{~mL})$ cooling in an ice-bath was treated with cyclohexanecarbonyl chloride $(2.71 \mathrm{~mL}$, $20.29 \mathrm{mmol}$ ). The mixture was stirred at $0{ }^{\circ} \mathrm{C}$ for 30 minutes and was allowed to warm to room temperature. Dichloromethane $(20 \mathrm{~mL})$ was added. The mixture was washed with saturated aqueous $\mathrm{NaHCO}_{3}$ and brine, and dried over $\mathrm{MgSO}_{4}$, filtered and concentrated. Purification via chromatography on an $80 \mathrm{~g}$ silica gel cartridge eluting with ethyl acetate in heptanes at 0-40\% gradient provided the title compound $6.2 \mathrm{~g}$ (92 \% yield). ${ }^{1} \mathrm{H}$ NMR $\left(400 \mathrm{MHz}, \mathrm{CDCl}_{3}\right) \delta \mathrm{ppm} 7.64(\mathrm{~d}, \mathrm{~J}=7.1 \mathrm{~Hz}, 2 \mathrm{H}), 7.42-7.31$ $(\mathrm{m}, 3 \mathrm{H}), 5.43(\mathrm{~d}, \mathrm{~J}=9.1 \mathrm{~Hz}, 1 \mathrm{H}), 5.34(\mathrm{dd}, \mathrm{J}=9.0,4.4 \mathrm{~Hz}, 1 \mathrm{H}), 4.79(\mathrm{~d}, \mathrm{~J}=4.6 \mathrm{~Hz}, 1 \mathrm{H}), 4.33(\mathrm{q}, \mathrm{J}=7.2$ $\mathrm{Hz}, 2 \mathrm{H}), 3.12(\mathrm{t}, \mathrm{J}=4.7 \mathrm{~Hz}, 1 \mathrm{H}), 2.03(\mathrm{dd}, \mathrm{J}=13.3,10.0 \mathrm{~Hz}, 1 \mathrm{H}), 1.85-1.65(\mathrm{~m}, 2 \mathrm{H}), 1.61(\mathrm{~d}, \mathrm{~J}=13.1$ Hz, 1H), $1.54-1.38$ (m, 4H), 1.35 (d, J = 7.2 Hz, 3H), 1.27 (d, J = 13.7 Hz, 1H), 1.15 (d, J = 14.5 Hz, 1H), $1.04(\mathrm{~s}, 9 \mathrm{H}), 0.56(\mathrm{q}, \mathrm{J}=13.2 \mathrm{~Hz}, 1 \mathrm{H})$; MS (ESI+) $\mathrm{m} / z 431(\mathrm{M}+\mathrm{H})$.

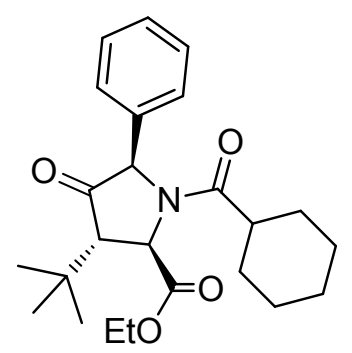

rac-(2R,3S,5R)-methyl 3-(tert-butyl)-1-(cyclohexanecarbonyl)-4-oxo-5-phenylpyrrolidine-2carboxylate (32)

Potassium dichromate $(5.11 \mathrm{~g}, 17.37 \mathrm{mmol})$ was dissolved in $6 \mathrm{M}$ aqueous $\mathrm{HCl}$ acid $(60 \mathrm{~mL})$ and zinc $(6$ g, $92 \mathrm{mmol}$ ) was added under $\mathrm{N}_{2}$ atmosphere. Complete dissolution of the zinc provided a clear light blue solution. The formed chromium(II) chloride was transferred to a refluxing solution of Compound 31 (1.1 $\mathrm{g}, 2.55 \mathrm{mmol})$ in ethanol $(60 \mathrm{~mL})$ under $\mathrm{N}_{2}$. The reaction mixture was refluxed for 16 hours. LC/MS indicated conversion was complete and two products were formed, one was desired product and another was hydrolyzed acid. The mixture was cooled to ambient temperature and extracted with ethyl acetate $(60 \mathrm{~mL} \times 3)$. The organics were washed with saturated aqueous $\mathrm{NaHCO}_{3}$ solution and brine, dried over $\mathrm{MgSO}_{4}$, filtered, and concentrated. The residue was dissolved in ethanol $(5 \mathrm{~mL})$, cooled in an ice-bath, and acetyl chloride $(1 \mathrm{~mL})$ in ethanol $(2 \mathrm{~mL})$ was slowly added. The mixture was stirred at $60^{\circ} \mathrm{C}$ for 2 hours and LC/MS indicated all acid was converted to the ester. The solvent was reduced in volume and the crude material was purified via chromatography on a $80 \mathrm{~g}$ silica gel cartridge, eluting with ethyl acetate in heptanes using a $0-40 \%$ gradient to provide the title compound ( $860 \mathrm{mg}, 86 \%$ yield). ${ }^{1} \mathrm{H}$ NMR 
$\left(400 \mathrm{MHz}, \mathrm{CDCl}_{3}\right) \delta \mathrm{ppm} 7.68(\mathrm{dd}, J=7.3,1.7 \mathrm{~Hz}, 2 \mathrm{H}), 7.45-7.38(\mathrm{~m}, 2 \mathrm{H}), 7.37-7.31(\mathrm{~m}, 1 \mathrm{H}), 4.90$ $(\mathrm{s}, 1 \mathrm{H}), 4.84(\mathrm{~d}, J=5.0 \mathrm{~Hz}, 1 \mathrm{H}), 4.28(\mathrm{q}, J=7.2 \mathrm{~Hz}, 2 \mathrm{H}), 2.54(\mathrm{dd}, J=5.0,1.0 \mathrm{~Hz}, 1 \mathrm{H}), 2.08$ (tt, $J=$ 11.4, $3.3 \mathrm{~Hz}, 1 \mathrm{H}), 1.70$ (t, $J=14.9 \mathrm{~Hz}, 2 \mathrm{H}), 1.54-1.48(\mathrm{~m}, 2 \mathrm{H}), 1.47-1.37$ (m, 2H), 1.32 (t, $J=7.2 \mathrm{~Hz}$, 3H), 1.10 (s, 9H), $0.92-0.82(\mathrm{~m}, 4 \mathrm{H})$; MS (ESI+) m/z 400.1 (M+H).

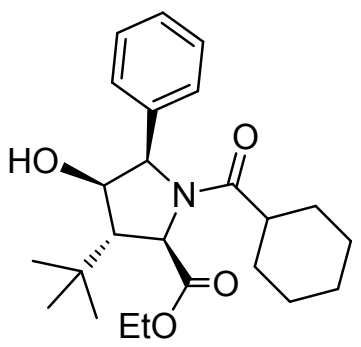

rac-(2R,3S,4R,5R)-ethyl 3-(tert-butyl)-1-(cyclohexanecarbonyl)-4-hydroxy-5-phenylpyrrolidine-2carboxylate (33)

A solution of Compound $32(200 \mathrm{mg}, 0.501 \mathrm{mmol})$ in methanol $(10 \mathrm{~mL})$ was cooled in an ice-bath and was treated with sodium borohydride $(37.9 \mathrm{mg}, 1.001 \mathrm{mmol})$. The mixture was stirred at $0{ }^{\circ} \mathrm{C}$ for 30 minutes, and was allowed to warm to ambient temperature. The solvent was removed and dichloromethane $(20 \mathrm{~mL})$ was added. The mixture was washed with saturated aqueous $\mathrm{NaHCO}_{3}$ and brine, dried over $\mathrm{Na}_{2} \mathrm{SO}_{4}$, filtered, and concentrated to provide title compound, $196 \mathrm{mg}$ (98\% yield) which used in next step without further purification. LC/MS (APCI+) $m / z 402(\mathrm{M}+\mathrm{H})^{+}$.

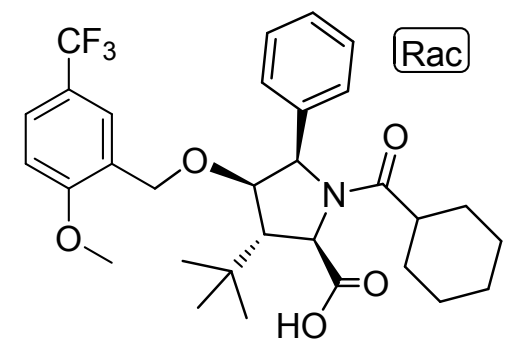

rac-(2R,3S,4R,5R)-3-tert-butyl-1-(cyclohexanecarbonyl)-4-\{[2-methoxy-5(trifluoromethyl)phenyl]methoxy\}-5-phenylpyrrolidine-2-carboxylic acid (5)

To 2-(bromomethyl)-1-methoxy-4-(trifluoromethyl)benzene (40 mg, $0.149 \mathrm{mmol}$ ) and Compound 33 (49.7 mg, $0.124 \mathrm{mmol})$ in dimethylformamide $(1.0 \mathrm{~mL})$ in an ice-bath was added sodium hydride $(7.43 \mathrm{mg}, 0.186 \mathrm{mmol})$ portionwise. The mixture was warmed to $60{ }^{\circ} \mathrm{C}$ and stirred for 3 hours. Ethyl acetate and water were added. The organic layer was washed with brine, dried over $\mathrm{MgSO}_{4}$, filtered, and concentrated. The residue was dissolved in methanol (2 $\mathrm{mL})$ and $6 \mathrm{M}$ aqueous $\mathrm{LiOH}(0.5 \mathrm{~mL})$ and stirred at $50{ }^{\circ} \mathrm{C}$ overnight. The mixture was adjusted 
to $\mathrm{pH} 1 \sim 2$ by adding $2 \mathrm{M}$ aqueous $\mathrm{HCl}$. The reaction mixture was extracted with ethyl acetate. The organic layers were combined, dried over sodium sulfate, and concentrated. The residue was purified via chromatography, eluting with ethyl acetate/methanol (9:1) in heptanes using a 0 $40 \%$ gradient to provide the title compound, $22 \mathrm{mg}$ (35\% yield). ${ }^{1} \mathrm{H}$ NMR ( $\left.400 \mathrm{MHz}, \mathrm{CDCl}_{3}\right) \delta$ ppm 7.42 - 7.37 (m, 1H), 7.33 (qd, J = 7.7, 6.7, 3.8 Hz, 3H), 7.25 - 7.20 (m, 2H), 6.81 - 6.75 (m, $2 \mathrm{H}), 5.17(\mathrm{~d}, \mathrm{~J}=6.6 \mathrm{~Hz}, 1 \mathrm{H}), 4.66(\mathrm{~d}, \mathrm{~J}=3.7 \mathrm{~Hz}, 1 \mathrm{H}), 4.56(\mathrm{~d}, \mathrm{~J}=13.3 \mathrm{~Hz}, 1 \mathrm{H}), 4.22$ - $4.11(\mathrm{~m}$, 2H), $3.77(\mathrm{~s}, 3 \mathrm{H}), 3.15(\mathrm{t}, \mathrm{J}=3.5 \mathrm{~Hz}, 1 \mathrm{H}), 2.35-2.23(\mathrm{~m}, 1 \mathrm{H}), 1.77(\mathrm{~d}, \mathrm{~J}=7.1 \mathrm{~Hz}, 1 \mathrm{H}), 1.68(\mathrm{~d}$, $\mathrm{J}=13.2 \mathrm{~Hz}, 2 \mathrm{H}), 1.55-1.41(\mathrm{~m}, 2 \mathrm{H}), 1.40-1.20(\mathrm{~m}, 2 \mathrm{H}), 1.14(\mathrm{t}, \mathrm{J}=10.5 \mathrm{~Hz}, 2 \mathrm{H}), 1.02(\mathrm{~s}, 9 \mathrm{H})$, $0.73(\mathrm{t}, \mathrm{J}=12.8 \mathrm{~Hz}, 1 \mathrm{H})$; MS (ESI-) $\mathrm{m} / z 560.3(\mathrm{M}-\mathrm{H})^{-}$.

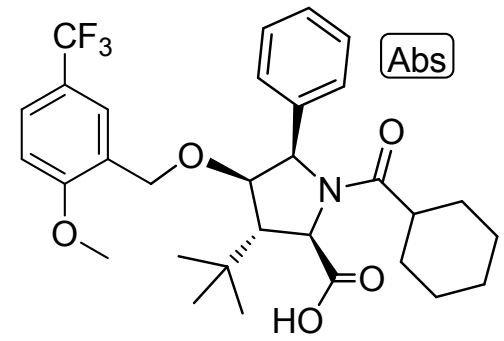

(2R,3S,4R,5R)-3-tert-butyl-1-(cyclohexanecarbonyl)-4-\{[2-methoxy-5-

(trifluoromethyl)phenyl]methoxy\}-5-phenylpyrrolidine-2-carboxylic acid (6)

Racemic compound 5 (6.55 g) was separated by chiral preparative SFC using a CHIRALPAK OZ-H, column size 30 x 250 mm, 5 micron, serial Number: OZH0SANG001-101201, using a concentration of $65 \mathrm{mg} / \mathrm{mL}$ in methanol with 2-propanol cosolvent (30\%) at a flow rate of $56 \mathrm{~g} / \mathrm{min} \mathrm{CO}_{2}$ to provide $2.10 \mathrm{~g}$ of the title compound. $\mathrm{R}_{\mathrm{T}}$ (chiral SFC) $=7.9 \mathrm{~min} ;{ }^{1} \mathrm{H}$ NMR $\left(400 \mathrm{MHz}, \mathrm{DMSO}-d_{6}, 120{ }^{\circ} \mathrm{C}\right) \delta \mathrm{ppm} 7.66(\mathrm{~d}$, $J=7.4 \mathrm{~Hz}, 2 \mathrm{H}), 7.44(\mathrm{dd}, J=8.8,2.4 \mathrm{~Hz}, 1 \mathrm{H}), 7.19(\mathrm{dt}, J=26.6,7.3 \mathrm{~Hz}, 3 \mathrm{H}), 7.07-6.89$ (m, 2H), 5.21 $(\mathrm{d}, J=6.4 \mathrm{~Hz}, 1 \mathrm{H}), 4.49$ (d, $J=3.4 \mathrm{~Hz}, 1 \mathrm{H}), 4.32(\mathrm{~d}, J=13.2 \mathrm{~Hz}, 1 \mathrm{H}), 4.25(\mathrm{dd}, J=6.5,2.7 \mathrm{~Hz}, 1 \mathrm{H})$, $3.98(\mathrm{~d}, J=13.2 \mathrm{~Hz}, 1 \mathrm{H}), 3.77(\mathrm{~s}, 3 \mathrm{H}), 2.51(\mathrm{~s}, 1 \mathrm{H}), 2.24(\mathrm{~s}, 1 \mathrm{H}), 1.65(\mathrm{~d}, J=9.8 \mathrm{~Hz}, 2 \mathrm{H}), 1.48(\mathrm{~s}, 2 \mathrm{H})$, $1.36-1.03(\mathrm{~m}, 5 \mathrm{H}), 0.99(\mathrm{~s}, 9 \mathrm{H}), 0.65-0.80(\mathrm{~m}, 1 \mathrm{H})$; MS (ESI+) $m / z 562.3(\mathrm{M}+\mathrm{H})^{+}$.

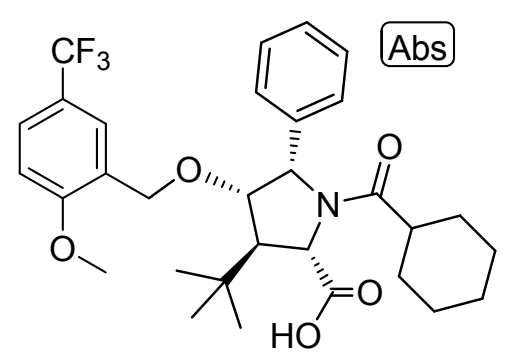




\section{(2S,3R,4S,5S)-3-tert-butyl-1-(cyclohexanecarbonyl)-4-\{[2-methoxy-5-}

\section{(trifluoromethyl)phenyl]methoxy\}-5-phenylpyrrolidine-2-carboxylic acid (7)}

Racemic compound 5 (6.55 g) was separated by chiral preparative SFC chromatography using a CHIRALPAK OZ-H, column size 30 x 250 mm, 5 micron, serial Number: OZH0SANG001-101201, using a concentration of $65 \mathrm{mg} / \mathrm{mL}$ in methanol with 2-propanol cosolvent (30\%) at a flow rate of 56 $\mathrm{g} / \mathrm{min} \mathrm{CO}_{2}$ and $\mathrm{UV}$ monitoring at $220 \mathrm{~nm}$ to provide $1.92 \mathrm{~g}$ of $(2 S, 3 R, 4 S, 5 S)-3$-(tert-butyl)-1(cyclohexanecarbonyl)-4-((2-methoxy-5-(trifluoromethyl)benzyl)oxy)-5-phenylpyrrolidine-2-carboxylic acid. The absolute structure of the title compound was determined by X-ray crystallography. $\mathrm{R}_{\mathrm{T}}$ (chiral $\mathrm{SFC})=6.0 \mathrm{~min} ;{ }^{1} \mathrm{H}$ NMR $\left(400 \mathrm{MHz}\right.$, DMSO- $\left.d_{6}, 120^{\circ} \mathrm{C}\right) \delta \mathrm{ppm} 7.66(\mathrm{~d}, J=7.4 \mathrm{~Hz}, 2 \mathrm{H}), 7.44(\mathrm{dd}, J=$ 8.8, 2.4 Hz, 1H), 7.19 (dt, $J=26.6,7.3 \mathrm{~Hz}, 3 \mathrm{H}), 7.07-6.89$ (m, 2H), 5.21 (d, $J=6.4 \mathrm{~Hz}, 1 \mathrm{H}), 4.49$ (d, $J$ $=3.4 \mathrm{~Hz}, 1 \mathrm{H}), 4.32(\mathrm{~d}, J=13.2 \mathrm{~Hz}, 1 \mathrm{H}), 4.25(\mathrm{dd}, J=6.5,2.7 \mathrm{~Hz}, 1 \mathrm{H}), 3.98(\mathrm{~d}, J=13.2 \mathrm{~Hz}, 1 \mathrm{H}), 3.77$ (s, $3 \mathrm{H}), 2.51(\mathrm{~s}, 1 \mathrm{H}), 2.24(\mathrm{~s}, 1 \mathrm{H}), 1.65(\mathrm{~d}, J=9.8 \mathrm{~Hz}, 2 \mathrm{H}), 1.48(\mathrm{~s}, 2 \mathrm{H}), 1.36-1.03(\mathrm{~m}, 5 \mathrm{H}), 0.99(\mathrm{~s}, 9 \mathrm{H})$, $0.60-0.81(\mathrm{~m}, 1 \mathrm{H}) ; \mathrm{MS}(\mathrm{ESI}+) \mathrm{m} / z 562.3(\mathrm{M}+\mathrm{H})^{+} ;[\alpha]^{24.8}=+83.9^{\circ}(c=0.85 \text {, methanol })^{+}$. 

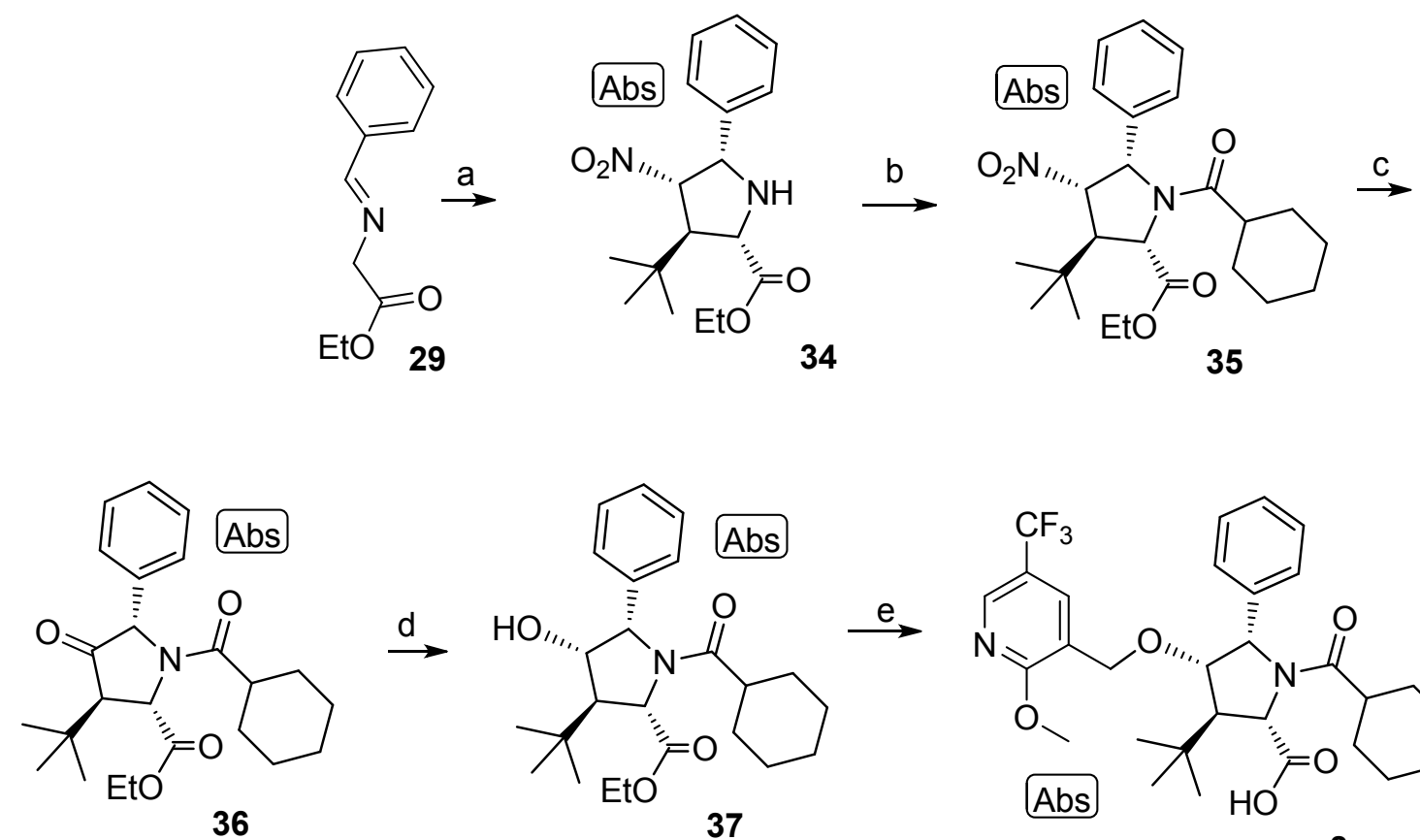<smiles>CCOC(=O)[C@@H]1[C@@H](C(C)(C)C)[C@@H](O)[C@H](c2ccccc2)N1C(=O)C1CCCCC1</smiles><smiles>COc1ncc(C(F)(F)F)cc1CO[C@H]1[C@@H](c2ccccc2)N(C(=O)C2CCCCC2)[C@H](C(=O)O)[C@H]1C(C)(C)C</smiles>

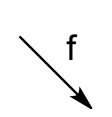<smiles>COc1ncc(C(C)(C)C)cc1CO[C@H]1[C@H](c2ccccc2)N(C(=O)C2CCCCC2)[C@H](C(=O)O)C1(C)C</smiles>

10

aReagents and conditions: (a) (2-(Bis(3,5-bis(trifluoromethyl)phenyl)phosphino)-3-((S)-4-isopropyl-4,5-dihydrooxazol-2yl)cyclopenta-2,4-dien-1-yl)(cyclopenta-2,4-dien-1-yl)iron, $\mathrm{Cu}(\mathrm{OTf})_{2}, \mathrm{KO} t \mathrm{Bu},(E)-3,3-$ dimethyl-1-nitrobut-1-ene, THF; (b) cyclohexanecarbonyl chloride, TEA, DCM; (c) PDC, 6N HCl, Zn dust, (d) NaBH 4 , EtOH; (e) 3-(bromomethyl)-2-methoxy-5(trifluoromethyl)pyridine, $\mathrm{KO} t \mathrm{Bu}, \mathrm{DMF}$; $\mathrm{LiOH}, \mathrm{MeOH}$; (f) 3-(bromomethyl)-5-(tert-butyl)-2-methoxypyridine, $\mathrm{KO} t \mathrm{Bu}, \mathrm{DMF}$; $\mathrm{LiOH}, \mathrm{MeOH}$.

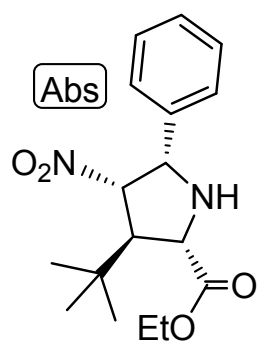

$(2 S, 3 R, 4 S, 5 S)$-ethyl 3-(tert-butyl)-4-nitro-5-phenylpyrrolidine-2-carboxylate (34) 
(2-(Bis(3,5-bis(trifluoromethyl)phenyl)phosphino)-3-((S)-4-isopropyl-4,5-dihydrooxazol-2yl)cyclopenta-2,4-dien-1-yl)(cyclopenta-2,4-dien-1-yl)iron (2.98 g, $3.96 \mathrm{mmol}$ ) and copper (I) triflate dimer, benzene complex ( $0.859 \mathrm{~g}, 1.707 \mathrm{mmol} ; 90 \%$ technical grade, Aldrich) were dissolved in tetrahydrofuran $(697 \mathrm{~mL})$ that had been sparged with a nitrogen stream for 2 hours. The resulting mixture was stirred for 90 minutes at ambient temperature, at which point the flask was cooled to an internal temperature below $5{ }^{\circ} \mathrm{C}$. (E)-Ethyl 2-(benzylideneamino)acetate (73.3 g, $383 \mathrm{mmol}$ ) was added in one portion via syringe. Potassium 2-methylpropan-2-olate (2.73 $\mathrm{mL}, 2.73 \mathrm{mmol}, 1 \mathrm{M}$ solution in tetrahydrofuran) was added dropwise, followed by addition of (E)-3,3-dimethyl-1-nitrobut-1-ene ( $45 \mathrm{~g}, 348 \mathrm{mmol}$ ) neat over 25 minutes via syringe, maintaining an internal temperature $<10^{\circ} \mathrm{C}$. After the addition was complete, the reaction was stirred for an additional 5 minutes at the same temperature, at which point LC-MS showed complete conversion of the starting nitroalkene. The reaction mixture was diluted with $300 \mathrm{~mL}$ of methyl tert-butyl ether and stirred with $300 \mathrm{~mL}$ of saturated aqueous ammonium chloride at ambient temperature for 15 minutes. The layers were separated, and the organic layer was washed with saturated aqueous ammonium chloride and brine and dried over sodium sulfate. After filtration, the organic extracts were concentrated in vacuo to provide a crude residue (140 g), which was precipitated from $800 \mathrm{~mL}$ of heptanes. The resulting material was removed via filtration using a fritted funnel, washed with $200 \mathrm{~mL}$ of cold heptanes, and dried to constant weight in a vacuum oven to provide $72.5 \mathrm{~g}(65 \%)$ of the title compound. ${ }^{1} \mathrm{H}$ NMR (400 MHz, $\left.\mathrm{CDCl}_{3}\right) \delta \mathrm{ppm} 7.37-7.18(\mathrm{~m}, 5 \mathrm{H}), 5.13(\mathrm{dd}, J=6.0,2.5 \mathrm{~Hz}, 1 \mathrm{H}), 4.45(\mathrm{dd}, J=12.4,6.0 \mathrm{~Hz}$, $1 \mathrm{H}), 4.32$ (qd, $J=7.2,1.2 \mathrm{~Hz}, 2 \mathrm{H}), 3.82(\mathrm{dd}, J=9.7,7.1 \mathrm{~Hz}, 1 \mathrm{H}), 3.30(\mathrm{dd}, J=12.3,9.8 \mathrm{~Hz}$, $1 \mathrm{H}), 2.96(\mathrm{dd}, J=7.2,2.5 \mathrm{~Hz}, 1 \mathrm{H}), 1.35$ (t, $J=7.1 \mathrm{~Hz}, 3 \mathrm{H}), 1.06$ (s, 9H); MS (ESI+) $m / z 321.1$ $(\mathrm{M}+\mathrm{H})^{+} ;[\alpha]^{24.8}=+16.1^{\circ}(c=1$, methanol $)$.

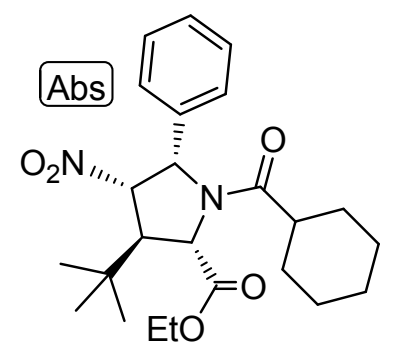

(2S,3R,4S,5S)-ethyl 3-(tert-butyl)-1-(cyclohexanecarbonyl)-4-nitro-5-phenylpyrrolidine-2carboxylate (35) 
To a cooled (ice bath) mixture of compound $34(2.0 \mathrm{~g}, 6.24 \mathrm{mmol})$ and triethylamine $(2.61 \mathrm{~mL}, 18.73$ $\mathrm{mmol})$ in dichloromethane $(20 \mathrm{~mL})$ was added cyclohexanecarbonyl chloride $(1.002 \mathrm{~mL}, 7.49 \mathrm{mmol})$ dropwise. The mixture was stirred in the ice-bath for 10 minutes and allowed to warm to room temperature. Dichloromethane $(10 \mathrm{~mL})$ was added. The mixture was washed with brine, dried over $\mathrm{MgSO}_{4}$, filtered, and concentrated to provide the title compound $(2.45 \mathrm{~g}, 91 \%) .{ }^{1} \mathrm{H} \mathrm{NMR}(400 \mathrm{MHz}$, $\left.\mathrm{CDCl}_{3}\right) \delta \mathrm{ppm} 7.73-7.61(\mathrm{~m}, 2 \mathrm{H}), 7.38(\mathrm{q}, \mathrm{J}=8.2,7.4 \mathrm{~Hz}, 3 \mathrm{H}), 5.46(\mathrm{~d}, \mathrm{~J}=9.0 \mathrm{~Hz}, 1 \mathrm{H}), 5.37(\mathrm{dd}, \mathrm{J}=$ 8.9, $4.5 \mathrm{~Hz}, 1 \mathrm{H}), 4.82(\mathrm{~d}, \mathrm{~J}=4.7 \mathrm{~Hz}, 1 \mathrm{H}), 4.36(\mathrm{q}, \mathrm{J}=7.2 \mathrm{~Hz}, 2 \mathrm{H}), 3.15(\mathrm{~d}, \mathrm{~J}=4.6 \mathrm{~Hz}, 1 \mathrm{H}), 2.06(\mathrm{ddt}, \mathrm{J}=$ 11.5, 7.0, 3.5 Hz, 1H), $1.68(\mathrm{dd}, \mathrm{J}=33.2,12.5 \mathrm{~Hz}, 2 \mathrm{H}), 1.55-1.41(\mathrm{~m}, 4 \mathrm{H}), 1.38(\mathrm{t}, \mathrm{J}=7.1 \mathrm{~Hz}, 3 \mathrm{H}), 1.34$ - $1.13(\mathrm{~m}, 3 \mathrm{H}), 1.07(\mathrm{~s}, 9 \mathrm{H}), 0.59(\mathrm{~d}, \mathrm{~J}=12.6 \mathrm{~Hz}, 1 \mathrm{H}) ; \mathrm{MS}(\mathrm{ESI}+) \mathrm{m} / z 431(\mathrm{M}+\mathrm{H})^{+}$.

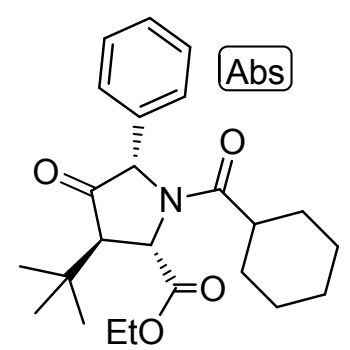

(2S,3R,5S)-ethyl 3-(tert-butyl)-1-(cyclohexanecarbonyl)-4-oxo-5-phenylpyrrolidine-2-carboxylate (36)

To potassium dichromate $(4.65 \mathrm{~g}, 15.79 \mathrm{mmol})$ in $6 \mathrm{M}$ aqueous $\mathrm{HCl}(60 \mathrm{~mL})$, zinc $(5.3 \mathrm{~g}, 81$ mmol) was added under $\mathrm{N}_{2}$ atmosphere. Complete dissolution of zinc provided a clear light blue solution. The formed chromium(II) chloride was transferred to the refluxing solution of compound 35 (1g, 2.323 mmol) in ethanol $(60 \mathrm{~mL})$. The reaction mixture was refluxed overnight. The mixture was cooled and concentrated to half of its volume and extracted with dichloromethane $(30 \mathrm{~mL} \times 3)$. The combined organic phase was washed with brine, dried over $\mathrm{MgSO}_{4}$, filtered, and concentrated. The residue was dissolved in ethanol $(2 \mathrm{~mL})$ and added to a prepared solution of acetyl chloride $(1 \mathrm{~mL})$ in ethanol $(4 \mathrm{~mL})$ cooling in an ice-bath. The mixture was heated to $60{ }^{\circ} \mathrm{C}$ for 2 hours, concentrated, and dissolved in ethyl acetate $(50 \mathrm{~mL})$ and saturated aqueous $\mathrm{NaHCO}_{3}(30 \mathrm{~mL})$. The organic layer was washed with brine, dried over $\mathrm{MgSO}_{4}$, filtered, and concentrated to provide a residue which purified via chromatography on a $40 \mathrm{~g}$ silica gel cartridge, eluting with ethyl acetate in heptanes at $0-30 \%$ gradient to provide the title compound (688 mg, $74.1 \%$ yield). 


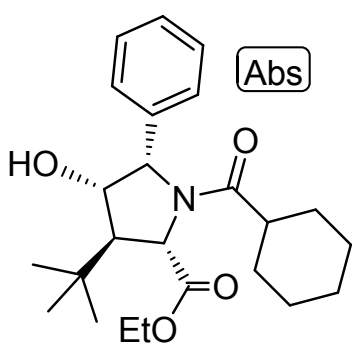

\section{(2S,3R,4S,5S)-ethyl 3-(tert-butyl)-1-(cyclohexanecarbonyl)-4-hydroxy-5-phenylpyrrolidine-2- carboxylate (37)}

Compound 36 (730 mg, $1.827 \mathrm{mmol})$ in ethanol $(10 \mathrm{~mL})$ cooled in an ice-bath was added sodium borohydride (138 mg, $3.65 \mathrm{mmol}$ ) portionwise. The mixture was stirred in ice-bath for 30 minutes and was allowed to warm to room temperature. LC/MS indicated the reaction was finished and showed two product peaks at ratio about 3 to 1, with the title compound as the major isomer. Saturated $\mathrm{NH}_{4} \mathrm{Cl}(2 \mathrm{~mL})$ was added, and the mixture was concentrated, dissolved in dichloromethane $(30 \mathrm{~mL})$, washed with brine, dried over $\mathrm{MgSO}_{4}$, filtered, and concentrated. Purification via chromatography on a $40 \mathrm{~g}$ silica gel cartridge eluting with ethyl acetate/methanol (9:1) in heptanes at $0-40 \%$ gradient provided the title compound (480 mg, $65.4 \%$ yield). LC/MS (APCI +$) m / z 402(\mathrm{M}+\mathrm{H})^{+}$

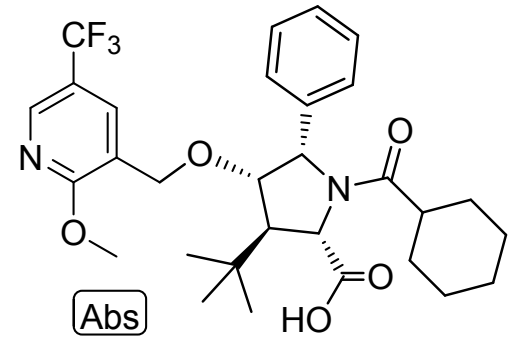

(2S,3R,4S,5S)-3-tert-butyl-1-(cyclohexanecarbonyl)-4-\{[2-methoxy-5-(trifluoromethyl)pyridin-3yl]methoxy\}-5-phenylpyrrolidine-2-carboxylic acid (8)

To compound 37 and 3-(bromomethyl)-2-methoxy-5-(trifluoromethyl)pyridine (121 mg, $0.448 \mathrm{mmol}$ ) in dimethylformamide $(2 \mathrm{~mL}$ ) cooled in an ice-bath, potassium 2-methylpropan-2-olate ( $62.9 \mathrm{mg}, 0.560$ mmol, $0.56 \mathrm{~mL}, 1.0 \mathrm{M}$ in tetrahydrofuran) was added dropwise. The mixture was stirred in the ice-bath for 20 minutes, and allowed to warm to room temperature. Methanol $(1.5 \mathrm{~mL})$ and $6 \mathrm{M}$ aqueous $\mathrm{LiOH}$ $(0.5 \mathrm{~mL})$ was added. The mixture was stirred at $50^{\circ} \mathrm{C}$ overnight, and adjusted to $\mathrm{pH} 1 \sim 2$ by adding $2 \mathrm{M}$ aqueous $\mathrm{HCl}$. The solvent was removed and dichloromethane was $(1 \mathrm{~mL})$ added. The reaction mixture was filtered through a syringe filter and the filtrate was purified via chromatography, eluting with methanol in dichloromethane at $0-20 \%$ gradient to provide title compound, $130 \mathrm{mg}(61.9 \%$ yield $) .{ }^{1} \mathrm{H}$ 
NMR (400 MHz, DMSO-d $\left.)_{6}\right) \delta$ ppm $8.29(\mathrm{td}, J=1.9,0.8 \mathrm{~Hz}, 1 \mathrm{H}), 7.66(\mathrm{~d}, J=7.3 \mathrm{~Hz}, 2 \mathrm{H}), 7.28-7.08$ (m, 4H), $5.23(\mathrm{~d}, J=6.3 \mathrm{~Hz}, 1 \mathrm{H}), 4.51(\mathrm{~d}, J=3.1 \mathrm{~Hz}, 1 \mathrm{H}), 4.38-4.24(\mathrm{~m}, 2 \mathrm{H}), 3.95(\mathrm{~d}, J=13.9 \mathrm{~Hz}, 1 \mathrm{H})$, $3.89(\mathrm{~s}, 3 \mathrm{H}), 2.51(\mathrm{~s}, 1 \mathrm{H}), 2.23(\mathrm{~s}, 1 \mathrm{H}), 1.65(\mathrm{~d}, J=9.5 \mathrm{~Hz}, 2 \mathrm{H}), 1.49(\mathrm{~s}, 2 \mathrm{H}), 1.17(\mathrm{~d}, J=73.3 \mathrm{~Hz}, 6 \mathrm{H})$, 1.01 (s, 9H); MS (ESI+) m/z $561.3(\mathrm{M}-\mathrm{H})^{-}$.

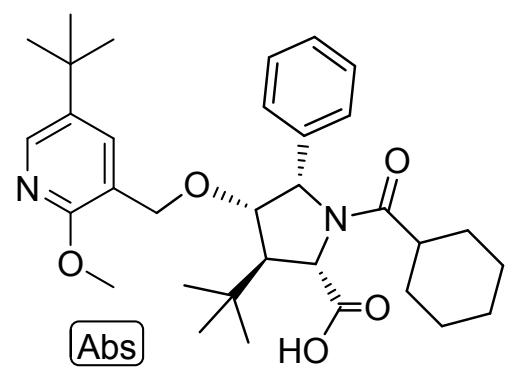

\section{(2S,3R,4S,5S)-3-tert-butyl-4-[(5-tert-butyl-2-methoxypyridin-3-yl)methoxy]-1-}

\section{(cyclohexanecarbonyl)-5-phenylpyrrolidine-2-carboxylic acid (10)}

The title compound was prepared according to the procedure described for compound $\mathbf{8}$, substituting 3(bromomethyl)-5-(tert-butyl)-2-methoxypyridine for 3-(bromomethyl)-2-methoxy-5(trifluoromethyl)pyridine. ${ }^{1} \mathrm{H}$ NMR $\left(400 \mathrm{MHz}, \mathrm{DMSO}-d_{6}\right) \delta \mathrm{ppm} 7.94(\mathrm{~d}, J=3.5 \mathrm{~Hz}, 1 \mathrm{H}), 7.68(\mathrm{~d}, J=$ $7.5 \mathrm{~Hz}, 2 \mathrm{H}), 7.30-7.10$ (m, 4H), 5.21 (d, $J=6.4 \mathrm{~Hz}, 1 \mathrm{H}), 4.47$ (d, $J=3.5 \mathrm{~Hz}, 1 \mathrm{H}), 4.23$ (dd, $J=11.2$, $5.5 \mathrm{~Hz}, 2 \mathrm{H}), 3.91(\mathrm{~d}, J=13.0 \mathrm{~Hz}, 1 \mathrm{H}), 3.77(\mathrm{~s}, 3 \mathrm{H}), 2.5(\mathrm{~m}, 1 \mathrm{H}), 2.24(\mathrm{~s}, 1 \mathrm{H}), 1.65(\mathrm{~d}, J=9.8 \mathrm{~Hz}, 2 \mathrm{H})$, 1.49 (s, 2H), 1.25 (d, $J=10.0 \mathrm{~Hz}, 2 \mathrm{H}), 1.21(\mathrm{~s}, 9 \mathrm{H}), 1.08$ (td, $J=10.1,9.5,4.0 \mathrm{~Hz}, 3 \mathrm{H}), 0.99$ (s, 9H), 0.87 -0.82 (m, 1H); MS (ESI-) $m / z 549.3(\mathrm{M}-\mathrm{H})^{-}$.

Scheme S4. Discovery Synthesis of Compound 9a
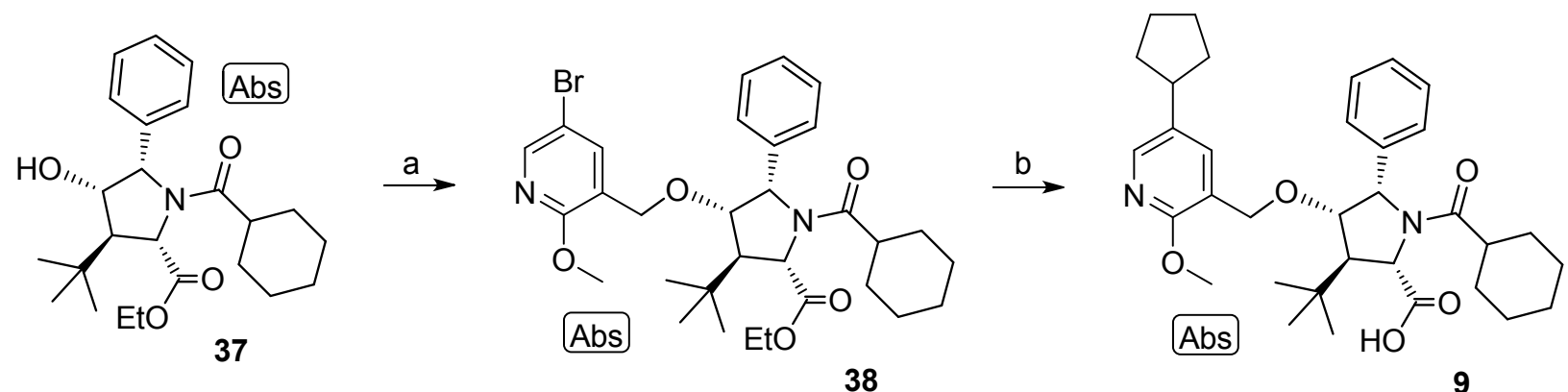

aReagents and conditions: (a) 5-bromo-3-(bromomethyl)-2-methoxypyridine; (e) PEPPSI-IPentCl, Cyclopentylzinc bromide; $\mathrm{LiOH}, \mathrm{THF} / \mathrm{MeOH}$. 


\section{(2S,3R,4S,5S)-ethyl 4-((5-bromo-2-methoxypyridin-3-yl)methoxy)-3-(tert-butyl)-1-}

\section{(cyclohexanecarbonyl)-5-phenylpyrrolidine-2-carboxylate (38)}

The title compound was prepared according to the procedure described for compound $\mathbf{8}$, substituting 5bromo-3-(bromomethyl)-2-methoxypyridine for 3-(bromomethyl)-2-methoxy-5-

(trifluoromethyl)pyridine. LC/MS (APCI+) $m / z 603.0(\mathrm{M}+\mathrm{H})^{+}$.

\section{(2S,3R,4S,5S)-3-tert-butyl-1-(cyclohexanecarbonyl)-4-[(5-cyclopentyl-2-methoxypyridin-3-}

\section{yl)methoxy]-5-phenylpyrrolidine-2-carboxylic acid (9)}

In a $4 \mathrm{~mL}$ vial, compound 38 (50.0 mg, $0.083 \mathrm{mmol}, 1.0 \mathrm{eq})$ and dichloro[4,5-dichloro-1,3-bis(2,6-Di-3pentylphenyl)imidazol-2-ylidene](3-chloropyridyl)palladium(II) (PEPPSI-IPentCl, $7.15 \mathrm{mg}, 0.0083$ mmol, $0.1 \mathrm{eq})$ were dissolved in tetrahydrofuran $(1.0 \mathrm{~mL})$, flushed with nitrogen, and stirred at room temperature. Cyclopentylzinc bromide $(0.5 \mathrm{M}, 498 \mu \mathrm{L}, 0.25 \mathrm{mmol}, 3.0 \mathrm{eq})$ was added and reaction was stirred at room temperature for 30 minutes. The solvent was removed under a stream of nitrogen, and the crude material was reconstituted in 3:2 tetrahydrofuran/methanol $(1.0 \mathrm{~mL})$. LiOH monohydrate $(34 \mathrm{mg}$, $0.83 \mathrm{mmol}, 10 \mathrm{eq})$ in $\mathrm{H}_{2} \mathrm{O}(300 \mu \mathrm{L})$ was added and reaction was stirred overnight at $45^{\circ} \mathrm{C}$. The solvent was removed under a stream of nitrogen. The residue was acidified with $1 \mathrm{M}$ aqueous $\mathrm{HCl}$ and extracted with dichloromethane $(3 \times 2 \mathrm{~mL})$. The solvent was removed and the crude material was reconstituted in acetonitrile. The reaction was loaded directly into an injection loop and purified using prep LC method TFA8 to provide the title compound $\left(36.0 \mathrm{mg}, 64.0 \%\right.$ yield). ${ }^{1} \mathrm{H}$ NMR $\left(400 \mathrm{MHz}, 120{ }^{\circ} \mathrm{C}, \mathrm{DMSO}-d_{6}\right.$ $\left.: \mathrm{D}_{2} \mathrm{O}=9: 1(\mathrm{v} / \mathrm{v})\right) \delta \mathrm{ppm} 7.78(\mathrm{~d}, \mathrm{~J}=2.4 \mathrm{~Hz}, 1 \mathrm{H}), 7.64(\mathrm{~d}, \mathrm{~J}=7.3 \mathrm{~Hz}, 2 \mathrm{H}), 7.28-7.13(\mathrm{~m}, 3 \mathrm{H}), 6.90(\mathrm{~d}, \mathrm{~J}$ $=2.4 \mathrm{~Hz}, 1 \mathrm{H}), 5.19(\mathrm{~d}, \mathrm{~J}=6.3 \mathrm{~Hz}, 1 \mathrm{H}), 4.49(\mathrm{~d}, \mathrm{~J}=3.3 \mathrm{~Hz}, 1 \mathrm{H}), 4.25-4.16(\mathrm{~m}, 2 \mathrm{H}), 3.90(\mathrm{~d}, \mathrm{~J}=13.0$ $\mathrm{Hz}, 1 \mathrm{H}), 3.76(\mathrm{~s}, 3 \mathrm{H}), 2.88-2.74(\mathrm{~m}, 1 \mathrm{H}), 2.50-2.46(\mathrm{~m}, 1 \mathrm{H}), 2.29-2.13(\mathrm{~m}, 1 \mathrm{H}), 1.95-1.87(\mathrm{~m}, 2 \mathrm{H})$, $1.81-1.70(\mathrm{~m}, 2 \mathrm{H}), 1.68-1.59(\mathrm{~m}, 4 \mathrm{H}), 1.50-1.45(\mathrm{~m}, 2 \mathrm{H}), 1.46-1.30(\mathrm{~m}, 2 \mathrm{H}), 1.27-1.03(\mathrm{~m}, 5 \mathrm{H})$, $0.98(\mathrm{~s}, 9 \mathrm{H}), 0.81-0.56(\mathrm{~m}, 1 \mathrm{H})$; MS (APCI+) $m / z 563.1(\mathrm{M}+\mathrm{H})^{+}$. 

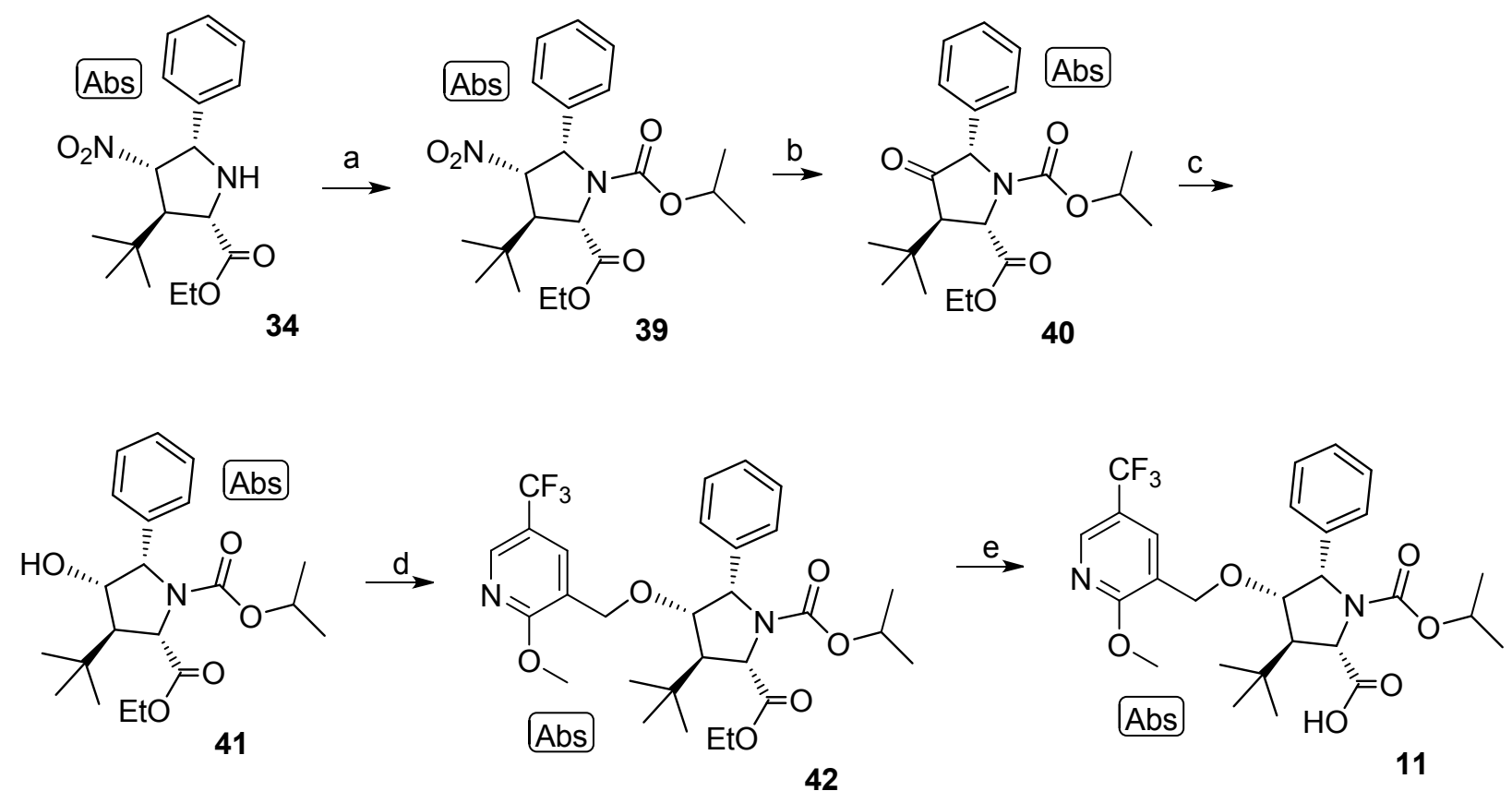

aReagents and conditions: (a) isopropyl chloroformate, TEA, DCM; (b) PDC, 6N HCl, Zn dust, (c) $\mathrm{NaBH}_{4}, \mathrm{EtOH}$; (d) 3(bromomethyl)-2-methoxy-5-(trifluoromethyl)pyridine, $\mathrm{KO} t \mathrm{Bu}, \mathrm{DMF}$; (e) $\mathrm{LiOH}(\mathrm{aq}), \mathrm{MeOH}$.

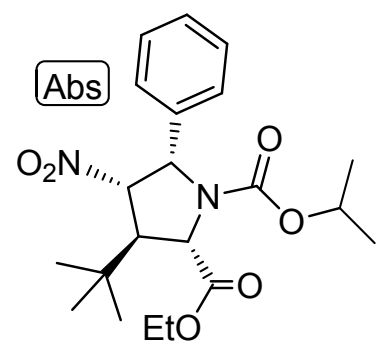

(2S,3R,4S,5S)-2-ethyl 1-isopropyl 3-(tert-butyl)-4-nitro-5-phenylpyrrolidine-1,2-dicarboxylate (39)

To a solution of compound $34(2.193 \mathrm{~g}, 6.84 \mathrm{mmol})$ in dichloromethane $(20 \mathrm{~mL})$ and triethylamine $(4 \mathrm{~mL}, 28.7 \mathrm{mmol})$ was added a $1 \mathrm{M}$ solution of isopropyl chloroformate $(15 \mathrm{~mL}$, $15.00 \mathrm{mmol}$ ) in toluene dropwise via addition funnel over about 3 minutes. After 30 minutes, additional $1 \mathrm{M}$ isopropyl chloroformate $(6 \mathrm{~mL})$ was added and after another 30 minutes additional $1 \mathrm{M}$ isopropyl chloroformate $(6 \mathrm{~mL})$ was added. The reaction mixture was stirred at room temperature for 3 hours more. The reaction mixture was diluted with dichloromethane $(200 \mathrm{~mL})$ and a small amount of ethanol and washed with saturated aqueous sodium bicarbonate $(50 \mathrm{~mL})$, dried over sodium sulfate, filtered, and concentrated. The crude material was purified using a $40 \mathrm{~g}$ silica gel cartridge with a gradient of 5-100\% ethyl acetate/heptanes over 30 minutes to provide $(2 S, 3 R, 4 S, 5 S)$-2-ethyl 1-isopropyl 3-(tert-butyl)-4-nitro-5-phenylpyrrolidine-1,2- 
dicarboxylate $\left(2.790 \mathrm{~g}, 6.88 \mathrm{mmol}, 100 \%\right.$ yield). ${ }^{1} \mathrm{H}$ NMR $\left(400 \mathrm{MHz}, 120^{\circ} \mathrm{C}\right.$, DMSO- $\left.d_{6}\right) \delta \mathrm{ppm}$ 7.56 - $7.46(\mathrm{~m}, 2 \mathrm{H}), 7.30$ - $7.15(\mathrm{~m}, 3 \mathrm{H}), 5.61(\mathrm{dd}, \mathrm{J}=8.8,3.3 \mathrm{~Hz}, 1 \mathrm{H}), 5.43(\mathrm{~d}, \mathrm{~J}=8.8 \mathrm{~Hz}, 1 \mathrm{H})$, $5.33(\mathrm{dd}, \mathrm{J}=6.6,3.2 \mathrm{~Hz}, 0 \mathrm{H}), 4.67(\mathrm{pd}, \mathrm{J}=6.1,0.7 \mathrm{~Hz}, 1 \mathrm{H}), 4.50(\mathrm{~d}, \mathrm{~J}=3.8 \mathrm{~Hz}, 1 \mathrm{H}), 4.25(\mathrm{qd}, \mathrm{J}$ $=7.1,0.6 \mathrm{~Hz}, 2 \mathrm{H}), 2.98(\mathrm{t}, \mathrm{J}=3.6 \mathrm{~Hz}, 1 \mathrm{H}), 1.29(\mathrm{td}, \mathrm{J}=7.0,0.7 \mathrm{~Hz}, 3 \mathrm{H}), 1.06(\mathrm{~d}, \mathrm{~J}=6.2 \mathrm{~Hz}$, $3 \mathrm{H}), 1.03$ - $0.98(\mathrm{~m}, 9 \mathrm{H}), 0.92(\mathrm{~d}, \mathrm{~J}=6.2 \mathrm{~Hz}, 3 \mathrm{H})$; MS (APCI+) $\mathrm{m} / \mathrm{z} 407(\mathrm{M}+\mathrm{H})^{+}$.

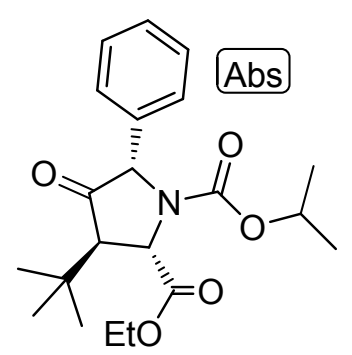

(2S,3R,5S)-2-ethyl 1-isopropyl 3-(tert-butyl)-4-oxo-5-phenylpyrrolidine-1,2-dicarboxylate (40)

Compound 39 (2.366 g, $5.82 \mathrm{mmol})$ was dissolved in ethanol $(75 \mathrm{~mL})$ and the solution was heated to $71^{\circ} \mathrm{C}$. A separate solution of $\mathrm{CrCl}_{2}$ was prepared by dissolving pyridinium dichromate (5.82 g, $19.79 \mathrm{mmol})$ in hydrochloric acid (6M, $75 \mathrm{~mL})$ and adding $\mathrm{Zn}(10.47 \mathrm{~g}, 160 \mathrm{mmol})$ in portions while cooling in an ice bath. The suspension was stirred until all almost all of the $\mathrm{Zn}$ dissolved, leaving a brilliant blue solution. The $\mathrm{CrCl}_{2}$ solution was transferred via cannula over 15 minutes to the solution of starting material (cooled to $65^{\circ} \mathrm{C}$ during the addition) and heating was continued at $80^{\circ} \mathrm{C}$ for 16 hours. The mixture was cooled to room temperature, reduced in volume in vacuo, diluted with water, and extracted with $3 \times 200 \mathrm{~mL}$ of dichloromethane. The combined extracts were washed with brine, dried over sodium sulfate, filtered, and concentrated in vacuo. The crude material was loaded onto a $80 \mathrm{~g}$ silica gel column and eluted with $0-100 \%$ ethyl acetate/heptanes over 40 minutes to provide ( $2 S, 3 R, 5 S)$-2-ethyl 1-isopropyl 3-(tert-butyl)4-oxo-5-phenylpyrrolidine-1,2-dicarboxylate (0.793 g, $2.112 \mathrm{mmol}, 36.3 \%$ yield). ${ }^{1} \mathrm{H}$ NMR $\left(400 \mathrm{MHz}, \mathrm{DMSO}-d_{6}\right) \delta \mathrm{ppm} 7.48$ - 7.40 (m, 2H), 7.33 - 7.27 (m, 2H), 7.26 - $7.19(\mathrm{~m}, 1 \mathrm{H}), 4.91$ (s, 1H), 4.77 - $4.69(\mathrm{~m}, 1 \mathrm{H}), 4.62(\mathrm{~d}, \mathrm{~J}=4.4 \mathrm{~Hz}, 1 \mathrm{H}), 4.19$ (qd, J = 7.1, 1.4 Hz, 2H), 2.56 (dd, J = 4.5, $0.9 \mathrm{~Hz}, 1 \mathrm{H}), 1.22$ (t, J = 7.1 Hz, 3H), 1.11 (d, J = 6.2 Hz, 3H), 1.05 (s, 9H), 0.98 (d, J = 6.2 $\mathrm{Hz}, 3 \mathrm{H}) ; \mathrm{MS}(\mathrm{APCI}+) \mathrm{m} / z 376(\mathrm{M}+\mathrm{H})^{+}$. 


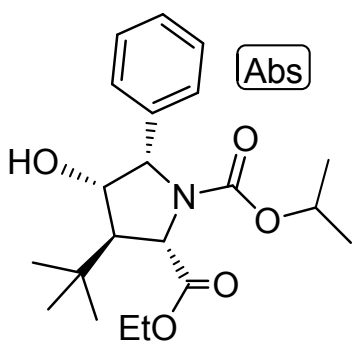

(2S,3R,4S,5S)-2-ethyl 1-isopropyl 3-(tert-butyl)-4-hydroxy-5-phenylpyrrolidine-1,2-dicarboxylate (41)

Compound 40 (0.611 g, $1.627 \mathrm{mmol})$ was dissolved in ethanol $(8.14 \mathrm{~mL})$ and sodium borohydride $(0.123$ $\mathrm{g}, 3.25 \mathrm{mmol}$ ) was added in one portion after cooling the reaction to $<5{ }^{\circ} \mathrm{C}$ in an ice-water bath. The reaction was stirred at the same temperature for 30 minutes, concentrated, and partitioned between ethyl acetate and saturated aqueous sodium bicarbonate. The organics were concentrated and purified using a $40 \mathrm{~g}$ silica gel cartridge and eluting with $0-100 \%$ ethyl acetate/heptanes over 40 minutes on to provide (2S,3R,4S,5S)-2-ethyl 1-isopropyl 3-(tert-butyl)-4-hydroxy-5-phenylpyrrolidine-1,2-dicarboxylate (0.506 g, 1.340 mmol, $82 \%$ yield). ${ }^{1} \mathrm{H}$ NMR (400 MHz, DMSO- $\left.d_{6}\right) \delta$ ppm 7.50 (dd, J=8.1,1.5 Hz, 2H), 7.19$7.27(\mathrm{~m}, 2 \mathrm{H}), 7.09-7.19(\mathrm{~m}, 1 \mathrm{H}), 4.82(\mathrm{~d}, \mathrm{~J}=6.7 \mathrm{~Hz}, 1 \mathrm{H}), 4.62(\mathrm{pd}, \mathrm{J}=6.2,1.1 \mathrm{~Hz}, 1 \mathrm{H}), 4.27-4.35(\mathrm{~m}, 1 \mathrm{H})$, $4.22(\mathrm{dd}, \mathrm{J}=4.8,1.2 \mathrm{~Hz}, 1 \mathrm{H}), 4.10-4.19(\mathrm{~m}, 2 \mathrm{H}), 3.84$ (d, J=7.4 Hz, 1H), 2.21-2.28 (m, 1H), 1.24 $(\mathrm{td}, \mathrm{J}=7.1,1.0 \mathrm{~Hz}, 3 \mathrm{H}), 1.04(\mathrm{dd}, \mathrm{J}=6.3,1.2 \mathrm{~Hz}, 3 \mathrm{H}), 0.98(\mathrm{~d}, \mathrm{~J}=1.1 \mathrm{~Hz}, 9 \mathrm{H}), 0.90$ (d, J=6.2 Hz, 3H); MS (APCI+) $m / z 378(\mathrm{M}+\mathrm{H})^{+}$.

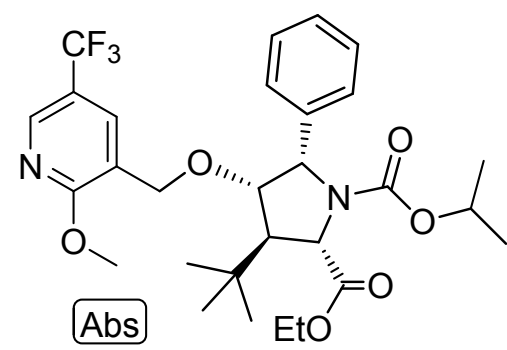

(2S,3R,4S,5S)-2-ethyl 1-isopropyl 3-(tert-butyl)-4-((2-methoxy-5-(trifluoromethyl)pyridin-3yl)methoxy)-5-phenylpyrrolidine-1,2-dicarboxylate (42)

Compound 41 (37 mg, $0.098 \mathrm{mmol}$ ) and 3-(bromomethyl)-2-methoxy-5-(trifluoromethyl)pyridine (47.6 $\mathrm{mg}, 0.176 \mathrm{mmol})$ were dissolved in dry dimethylformamide $(0.5 \mathrm{~mL})$. After cooling in an ice bath, potassium 2-methylpropan-2-olate $(0.157 \mathrm{~mL}, 0.157 \mathrm{mmol})$ solution was added dropwise over 2 minutes. LC/MS showed desired product. The mixture was acidified with $1 \mathrm{M}$ aqueous $\mathrm{HCl}$ (10 drops) and warmed to room temperature. The mixture was concentrated and loaded onto a $12 \mathrm{~g}$ silica gel column and eluted with 5-100\% ethyl acetate/heptanes over 20 minutes to provide (2S,3R,4S,5S)-2-ethyl 1isopropyl 3-(tert-butyl)-4-((2-methoxy-5-(trifluoromethyl)pyridin-3-yl)methoxy)-5-phenylpyrrolidine- 
1,2-dicarboxylate (52 mg, $0.092 \mathrm{mmol}, 94 \%$ yield). ${ }^{1} \mathrm{H}$ NMR (400 MHz, DMSO- $\left.d_{6}\right) \delta \mathrm{ppm} 8.28(\mathrm{~s}, 1 \mathrm{H})$, 7.58 - $7.53(\mathrm{~m}, 2 \mathrm{H}), 7.22(\mathrm{~d}, \mathrm{~J}=2.3 \mathrm{~Hz}, 1 \mathrm{H}), 7.17(\mathrm{dd}, \mathrm{J}=8.2,6.6 \mathrm{~Hz}, 2 \mathrm{H}), 7.13-7.07(\mathrm{~m}, 1 \mathrm{H}), 5.02(\mathrm{~d}, \mathrm{~J}$ $=6.1 \mathrm{~Hz}, 1 \mathrm{H}), 4.64(\mathrm{pd}, \mathrm{J}=6.2,0.9 \mathrm{~Hz}, 1 \mathrm{H}), 4.37(\mathrm{~d}, \mathrm{~J}=3.1 \mathrm{~Hz}, 1 \mathrm{H}), 4.30$ - $4.24(\mathrm{~m}, 2 \mathrm{H}), 4.16$ - $4.03(\mathrm{~m}$, 2H), $3.87(\mathrm{~d}, \mathrm{~J}=0.9 \mathrm{~Hz}, 3 \mathrm{H}), 2.87-2.81(\mathrm{~m}, 2 \mathrm{H}), 1.15(\mathrm{td}, \mathrm{J}=7.1,0.9 \mathrm{~Hz}, 3 \mathrm{H}), 1.05(\mathrm{~d}, \mathrm{~J}=6.2 \mathrm{~Hz}, 3 \mathrm{H})$, $1.01(\mathrm{~d}, \mathrm{~J}=0.9 \mathrm{~Hz}, 9 \mathrm{H}), 0.90(\mathrm{~d}, \mathrm{~J}=6.2 \mathrm{~Hz}, 3 \mathrm{H}) ; \mathrm{MS}(\mathrm{APCI}+) \mathrm{m} / z 567(\mathrm{M}+\mathrm{H})^{+}$.

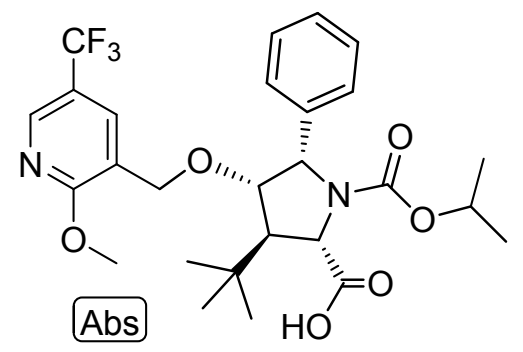

\section{$(2 S, 3 R, 4 S, 5 S)-3-t e r t-b u t y l-4-\{[2-m e t h o x y-5-(t r i f l u o r o m e t h y l) p y r i d i n-3-y l] m e t h o x y\}-5-p h e n y l-1-$}

\section{$\{[($ propan-2-yl)oxy]carbonyl\}pyrrolidine-2-carboxylic acid (11)}

Compound 42 (52 mg, $0.092 \mathrm{mmol})$ was dissolved in methanol $(0.5 \mathrm{~mL})$ and a $2 \mathrm{M}$ aqueous solution of lithium hydroxide $(0.459 \mathrm{~mL}, 0.918 \mathrm{mmol})$ was added. The reaction was warmed at $45{ }^{\circ} \mathrm{C}$ overnight, acidified with $2 \mathrm{M}$ aqueous $\mathrm{HCl}(0.460 \mathrm{~mL})$, and concentrated. The residue was loaded onto a $12 \mathrm{~g}$ silica gel column and eluted with 5-100\% ethyl acetate/ethanol/heptanes over 20 minutes to provide (2S,3R,4S,5S)-3-(tert-butyl)-1-(isopropoxycarbonyl)-4-((2-methoxy-5-(trifluoromethyl)pyridin-3yl)methoxy)-5-phenylpyrrolidine-2-carboxylic acid (22 mg, $0.041 \mathrm{mmol}, 44.5 \%$ yield). ${ }^{1} \mathrm{H}$ NMR (400 MHz, DMSO- $\left.d_{6}\right) \delta$ ppm 7.68 - $7.62(\mathrm{~m}, 2 \mathrm{H}), 7.20$ - $7.16(\mathrm{~m}, 2 \mathrm{H}), 7.16$ - $7.12(\mathrm{~m}, 2 \mathrm{H}), 7.13$ - $7.05(\mathrm{~m}$, $1 \mathrm{H}), 5.00(\mathrm{~d}, \mathrm{~J}=6.5 \mathrm{~Hz}, 1 \mathrm{H}), 4.63(\mathrm{p}, \mathrm{J}=6.2 \mathrm{~Hz}, 1 \mathrm{H}), 4.30(\mathrm{~d}, \mathrm{~J}=14.0 \mathrm{~Hz}, 1 \mathrm{H}), 4.26(\mathrm{~d}, \mathrm{~J}=3.1 \mathrm{~Hz}, 1 \mathrm{H})$, $4.21(\mathrm{dd}, \mathrm{J}=6.5,2.5 \mathrm{~Hz}, 1 \mathrm{H}), 3.93(\mathrm{~d}, \mathrm{~J}=13.9 \mathrm{~Hz}, 1 \mathrm{H}), 3.88(\mathrm{~s}, 3 \mathrm{H}), 2.55(\mathrm{t}, \mathrm{J}=2.8 \mathrm{~Hz}, 1 \mathrm{H}), 1.06(\mathrm{~d}, \mathrm{~J}=$ $6.2 \mathrm{~Hz}, 3 \mathrm{H}), 1.00(\mathrm{~s}, 9 \mathrm{H}), 0.89(\mathrm{~d}, \mathrm{~J}=6.2 \mathrm{~Hz}, 3 \mathrm{H})$; MS (APCI+) $m / z 539.2(\mathrm{M}+\mathrm{H})^{+}$. 
<smiles>C=CCOC(=O)N1[C@H](C(=O)OCC)C(C(C)(C)C)[C@@H](C(C)C)[C@H]1c1ccccc1</smiles><smiles>C=CCOC(=O)N1[C@H](C(=O)OCC)[C@@H](C(C)(C)C)[C@H](O)[C@H]1c1ccccc1</smiles><smiles>CCOC(=O)[C@H]1[C@@H](C(C)(C)C)[C@@H](OCc2cc(C(F)(F)F)cnc2OC)[C@H](c2ccccc2)N1C(=O)[C@@H]1CCCCO1</smiles><smiles>C1C[Te]C1</smiles><smiles>CCOC(=O)[C@H]1[C@@H](C(C)(C)C)[C@@H](OCc2cc(C(F)(F)F)cnc2OC)[C@H](c2ccccc2)N1C(=O)C1CCCCO1</smiles>

$g \downarrow$<smiles>COc1ncc(C(F)(F)F)cc1CO[C@@H]1[C@H](C(C)(C)C)N(C(=O)[C@@H]2CCCCO2)[C@H](C(=O)O)[C@H]1c1ccccc1</smiles><smiles>COc1ncc(C(F)(F)F)cc1CO[C@@H]1[C@H](c2ccccc2)N(C(=O)[C@@H]2CCCCO2)[C@H](C(=O)O)[C@@H]1C(C)(C)C</smiles>

aReagents and conditions: (a) allyl chloroformate, TEA, DCM; (b) PDC, 6N HCl, Zn dust, (c) $\mathrm{NaBH}_{4}$, EtOH; (d) 3(bromomethyl)-2-methoxy-5-(trifluoromethyl)pyridine, KOtBu, DMF; (e) 1,3-Dimethlbarbituric acid, $\mathrm{Pd}\left(\mathrm{PPh}_{3}\right)_{4}, \mathrm{EtOAc} / \mathrm{DCM}$; (f) Tetrahydropyran-2-carboxylic acid, oxalyl chloride, DMF, DCM; (g) LiOH (aq), $\mathrm{MeOH}$. 


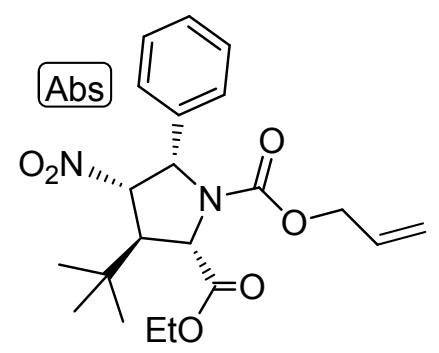

(2S,3R,4S,5S)-1-allyl 2-ethyl 3-(tert-butyl)-4-nitro-5-phenylpyrrolidine-1,2-dicarboxylate (43)

To compound $34(1.0 \mathrm{~g}, 3.12 \mathrm{mmol})$ in toluene $(3 \mathrm{~mL})$ and saturated $\mathrm{NaHCO}_{3}(3.00 \mathrm{~mL})$ was added allyl cahloroformate $(0.332 \mathrm{~mL}, 3.12 \mathrm{mmol})$ dropwise at ambient temperature, and the mixture was stirred for 30 minutes. LC/MS indicated the conversion was finished. Dichloromethane $(20 \mathrm{~mL})$ and water $(10 \mathrm{~mL})$ were added and the organic layer washed with brine, dried over $\mathrm{MgSO}_{4}$, filtered, and concentrated. The residue was purified via silica gel chromatography, eluting with ethyl acetate in hepetane at $0-40 \%$ gradient to provide the title compound $\left(1.0 \mathrm{~g}, 95 \%\right.$ yield). ${ }^{1} \mathrm{H}$ NMR $\left(501 \mathrm{MHz}, \mathrm{CDCl}_{3}\right) \delta \mathrm{ppm} 7.58$ $7.51(\mathrm{~m}, 2 \mathrm{H}), 7.37$ - $7.26(\mathrm{~m}, 3 \mathrm{H}), 5.61(\mathrm{~s}, 2 \mathrm{H}), 5.43(\mathrm{~d}, \mathrm{~J}=8.7 \mathrm{~Hz}, 1 \mathrm{H}), 5.30$ (dd, J = 8.7, 2.6 Hz, 1H), $5.00(\mathrm{~s}, 1 \mathrm{H}), 4.70(\mathrm{~s}, 1 \mathrm{H}), 4.56(\mathrm{~s}, 2 \mathrm{H}), 4.39(\mathrm{qd}, \mathrm{J}=7.1,1.8 \mathrm{~Hz}, 2 \mathrm{H}), 3.11(\mathrm{t}, \mathrm{J}=2.9 \mathrm{~Hz}, 1 \mathrm{H}), 1.40(\mathrm{t}, \mathrm{J}=$ $7.1 \mathrm{~Hz}, 3 \mathrm{H}), 1.10(\mathrm{~s}, 9 \mathrm{H})$; MS (ESI+) $m / z 405(\mathrm{M}+\mathrm{H})^{+}$.

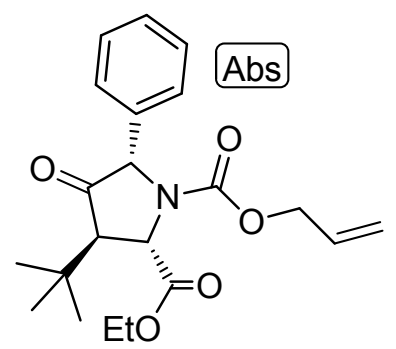

(2S,3R,5S)-1-allyl 2-ethyl 3-(tert-butyl)-4-oxo-5-phenylpyrrolidine-1,2-dicarboxylate (44)

Compound $\mathbf{4 4}$ was prepared according to the procedure described to prepare compound 24 from compound 23. LC/MS (ESI+) $=374.45(\mathrm{M}+\mathrm{H})^{+}$.<smiles>C=CCOC(=O)N1[C@H](C(=O)OCC)[C@@H](O)[C@H](C(C)(C)C)[C@H]1c1ccccc1</smiles>

$(2 S, 3 R, 4 S, 5 S)$-1-allyl 2-ethyl 3-(tert-butyl)-4-hydroxy-5-phenylpyrrolidine-1,2-dicarboxylate (45) 
To compound 44 (560 mg, $1.5 \mathrm{mmol})$ in ethanol (4 mL) cooling in an ice-bath was added sodium borohydride $(113 \mathrm{mg}, 3.00 \mathrm{mmol})$ portionwise. The mixture was stirred in the ice-bath for 30 minutes, and allowed to warm to ambient temperature. LC/MS showed two product peaks, in a ratio of about 6 to 1. The solvent was removed, dichloromethane $(20 \mathrm{~mL})$ was added and the organics were washed with brine. The organics were dried over $\mathrm{MgSO}_{4}$, filtered, and concentrated. Purification via chromatography, eluting with ethyl acetate/methanol (9:1) in heptanes at 0-40\% gradient provided title compound as the second eluent (340 mg, $60 \%$ yield). ${ }^{1} \mathrm{H}$ NMR (400 MHz, $\left.\mathrm{CDCl}_{3}\right) \delta$ ppm $7.64-7.56$ (m, 2H), $7.43-7.36$ $(\mathrm{m}, 2 \mathrm{H}), 7.33-7.28(\mathrm{~m}, 1 \mathrm{H}), 5.55(\mathrm{~s}, 1 \mathrm{H}), 5.04(\mathrm{~s}, 1 \mathrm{H}), 4.97-4.70(\mathrm{~m}, 1 \mathrm{H}), 4.64-4.38(\mathrm{~m}, 3 \mathrm{H}), 4.35-$ $4.31(\mathrm{~m}, 1 \mathrm{H}), 4.27(\mathrm{ddd}, J=10.8,7.0,3.4 \mathrm{~Hz}, 2 \mathrm{H}), 2.41-2.32(\mathrm{~m}, 1 \mathrm{H}), 1.47$ (d, $J=5.0 \mathrm{~Hz}, 1 \mathrm{H}), 1.34(\mathrm{t}$, $J=7.1 \mathrm{~Hz}, 3 \mathrm{H}), 1.07(\mathrm{~s}, 9 \mathrm{H}) ; \mathrm{LC} / \mathrm{MS}(\mathrm{ESI}+) \mathrm{m} / z 376.5(\mathrm{M}+\mathrm{H})^{+}$. Also obtained was the other diastereomer (2S,3R,4R,5S)-1-allyl 2-ethyl 3-(tert-butyl)-4-hydroxy-5-phenylpyrrolidine-1,2dicarboxylate as the first eluting isomer (56 $\mathrm{mg}, 10 \%$ yield).

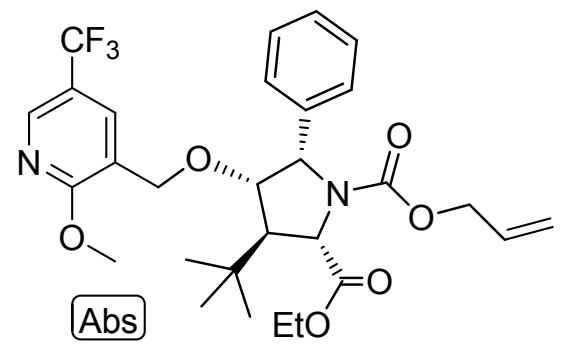

\section{(2S,3R,4S,5S)-1-allyl 2-ethyl 3-(tert-butyl)-4-((2-methoxy-5-(trifluoromethyl)pyridin-3-yl)methoxy)-}

\section{5-phenylpyrrolidine-1,2-dicarboxylate (46)}

To compound 45 (340 mg, $0.906 \mathrm{mmol}$ ) and 3-(bromomethyl)-2-methoxy-5-(trifluoromethyl)pyridine $(293 \mathrm{mg}, 1.087 \mathrm{mmol})$ in dimethylformamide $(2 \mathrm{~mL})$ in an ice-bath was added potassium 2methylpropan-2-olate (152 mg, $1.358 \mathrm{mmol}, 1.6 \mathrm{~mL}, 1.0 \mathrm{M}$ in tetrahydrofuran) dropwise. The mixture was stirred in ice-bath for 20 minutes, and allowed to warm to room temperature. Saturated aqueous $\mathrm{NH}_{4} \mathrm{Cl}(2 \mathrm{~mL})$ was added, and dichloromethane $(20 \mathrm{~mL})$ was added. The mixture was washed with brine, dried over $\mathrm{MgSO}_{4}$, filtered, and concentrated. Purification of the residue via chromatography, eluting with ethyl acetate/methanol (9:1) in heptanes at $0-40 \%$ gradient provided the title compound (380 $\mathrm{mg}, 71$ \% yield). ${ }^{1} \mathrm{H}$ NMR (400 MHz, DMSO- $\left.d_{6}\right) \delta \mathrm{ppm} 8.29(\mathrm{t}, J=1.5 \mathrm{~Hz}, 1 \mathrm{H}), 7.62-7.52(\mathrm{~m}, 2 \mathrm{H}), 7.26-$ $7.15(\mathrm{~m}, 3 \mathrm{H}), 7.13-7.05(\mathrm{~m}, 1 \mathrm{H}), 5.73-5.66(\mathrm{~m}, 1 \mathrm{H}), 5.08$ (d, $J=6.1 \mathrm{~Hz}, 1 \mathrm{H}), 5.04$ (dq, $J=3.2,1.8$ $\mathrm{Hz}, 1 \mathrm{H}), 5.01(\mathrm{t}, J=1.7 \mathrm{~Hz}, 1 \mathrm{H}), 4.41(\mathrm{ddd}, J=5.1,3.6,2.3 \mathrm{~Hz}, 3 \mathrm{H}), 4.30-4.25(\mathrm{~m}, 2 \mathrm{H}), 4.10(\mathrm{qd}, J=$ 7.1, $2.3 \mathrm{~Hz}, 2 \mathrm{H}), 3.87(\mathrm{~s}, 3 \mathrm{H}), 1.15(\mathrm{t}, J=7.0 \mathrm{~Hz}, 3 \mathrm{H}), 1.01(\mathrm{~s}, 9 \mathrm{H})$; MS (ESI+) $m / z 565(\mathrm{M}+\mathrm{H})^{+}$. 


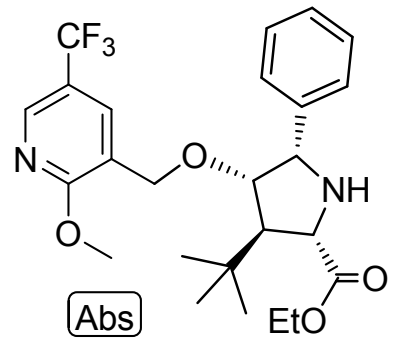

$(2 S, 3 R, 4 S, 5 S)$-ethyl 3-(tert-butyl)-4-((2-methoxy-5-(trifluoromethyl)pyridin-3-yl)methoxy)-5phenylpyrrolidine-2-carboxylate (47)

To compound $46(320 \mathrm{mg}, 0.567 \mathrm{mmol})$ in a mixture of acetonitrile and water $(3.3 \mathrm{~mL}, 10: 1)$ was added diethylamine $(0.117 \mathrm{~mL}, 1.134 \mathrm{mmol})$ and tetrakis(triphenylphosphine) palladium(0) (14.41 mg, $0.012 \mathrm{mmol}$ ). The mixture was stirred at ambient temperature overnight.

Dichloromethane $(10 \mathrm{~mL})$ and water $(10 \mathrm{~mL})$ were added. The organic layer was washed with brine, dried over magnesium sulfate, filtered, and concentrated. Purification via chromatography, eluting with ethyl acetate/methanol (10:1) in heptanes at 0-50\% gradient provided the title compound (260 mg, $95 \%$ yield). ${ }^{1} \mathrm{H} \mathrm{NMR}\left(400 \mathrm{MHz}, \mathrm{CDCl}_{3}\right) \delta \mathrm{ppm} 8.24(\mathrm{dt}$, $J=1.9,0.9 \mathrm{~Hz}, 1 \mathrm{H}), 7.46-7.41(\mathrm{~m}, 2 \mathrm{H}), 7.37(\mathrm{~d}, J=2.4 \mathrm{~Hz}, 1 \mathrm{H}), 7.35-7.29(\mathrm{~m}, 2 \mathrm{H}), 7.28-$ $7.23(\mathrm{~m}, 1 \mathrm{H}), 4.33(\mathrm{dt}, J=14.0,0.9 \mathrm{~Hz}, 1 \mathrm{H}), 4.25(\mathrm{qd}, J=7.2,0.8 \mathrm{~Hz}, 2 \mathrm{H}), 4.17$ (d, $J=4.1 \mathrm{~Hz}$, 1H), 3.94 (dd, $J=4.2,1.4 \mathrm{~Hz}, 1 \mathrm{H}), 3.93-3.88(\mathrm{~m}, 4 \mathrm{H}), 3.74$ (d, $J=6.2 \mathrm{~Hz}, 1 \mathrm{H}), 2.85$ (s, 1H), $2.43(\mathrm{dd}, J=6.3,1.3 \mathrm{~Hz}, 1 \mathrm{H}), 1.30(\mathrm{t}, J=7.1 \mathrm{~Hz}, 3 \mathrm{H}), 1.06(\mathrm{~s}, 9 \mathrm{H})$; MS (ESI+) $m / z 563(\mathrm{M}+\mathrm{H})^{+}$.<smiles>CCOC(=O)[C@H]1[C@@H](C(C)(C)C)[C@@H](OCc2cc(C(F)(F)F)cnc2OC)[C@H](c2ccccc2)N1C(=O)[C@@H]1CCCCO1</smiles>

(2S,3R,4S,5S)-ethyl 3-(tert-butyl)-4-((2-methoxy-5-(trifluoromethyl)pyridin-3-yl)methoxy)-5-phenyl1-((S)-tetrahydro-2H-pyran-2-carbonyl)pyrrolidine-2-carboxylate (48) and

(2S,3R,4S,5S)-ethyl 3-(tert-butyl)-4-((2-methoxy-5-(trifluoromethyl)pyridin-3-yl)methoxy)-5phenyl-1-((R)-tetrahydro-2H-pyran-2-carbonyl)pyrrolidine-2-carboxylate (49)

To tetrahydro-2H-pyran-2-carboxylic acid $(65.0 \mathrm{mg}, 0.499 \mathrm{mmol})$ in dichloromethane $(6 \mathrm{~mL})$ and a drop of dimethylformamide was added oxalyl dichloride (127 mg, $1.0 \mathrm{mmol}, 0.5 \mathrm{~mL}, 2 \mathrm{M}$ in dichloromethane). The mixture was stirred for 30 minutes and the solvent was removed under pressure and fresh 
dichloromethane was added and removed again. The residue was dissolved in dichloromethane $(1 \mathrm{~mL})$ and was added dropwise to the solution of compound $47(120 \mathrm{mg}, 0.250 \mathrm{mmol})$ and triethylamine $(0.139$ $\mathrm{mL}, 1.0 \mathrm{mmol})$ in dichloromethane $(6 \mathrm{~mL})$ cooled with an ice-bath. The mixture was stirred in an icebath for 30 minutes and was warmed to room temperature. LC/MS indicated the reaction was finished and showed two diasteiroisomers peaks at a ratio of about 1:1. Saturated aqueous $\mathrm{NH}_{4} \mathrm{Cl}(2 \mathrm{~mL})$ and dichloromethane $(10 \mathrm{~mL})$ were added. The organic layer was washed with brine, dried over $\mathrm{MgSO}_{4}$, filtered, and concentrated. Purification via chromatography on a $24 \mathrm{~g}$ silica gel cartridge, eluting with ethyl acetate in heptanes at 0-40\% gradient provided compound $\mathbf{4 8}$ as the first eluting isomer (55 $\mathrm{mg}, 37$ \% yield). ${ }^{1} \mathrm{H}$ NMR (400 MHz, $\left.\mathrm{CDCl}_{3}\right) \delta$ ppm 8.29 - $8.16(\mathrm{~m}, 1 \mathrm{H}), 7.75$ - 7.61 (m, 2H), 7.40 - 7.24 (m, $3 \mathrm{H}), 7.12(\mathrm{~d}, \mathrm{~J}=2.3 \mathrm{~Hz}, 1 \mathrm{H}), 5.52(\mathrm{~d}, \mathrm{~J}=6.1 \mathrm{~Hz}, 1 \mathrm{H}), 4.74(\mathrm{~d}, \mathrm{~J}=3.1 \mathrm{~Hz}, 1 \mathrm{H}), 4.32(\mathrm{~d}, \mathrm{~J}=14.1 \mathrm{~Hz}, 1 \mathrm{H})$, 4.21 (qq, J = 7.1, $3.7 \mathrm{~Hz}, 2 \mathrm{H}), 4.15-4.06(\mathrm{~m}, 1 \mathrm{H}), 3.91(\mathrm{~s}, 3 \mathrm{H}), 3.85$ (dd, J = 13.7, $4.5 \mathrm{~Hz}, 2 \mathrm{H}), 3.38$ (dd, $\mathrm{J}=10.3,2.7 \mathrm{~Hz}, 1 \mathrm{H}), 2.95(\mathrm{td}, \mathrm{J}=11.5,2.5 \mathrm{~Hz}, 1 \mathrm{H}), 2.51$ (t, J = 2.7 Hz, 1H), 1.77 - 1.29 (m, 6H), 1.26 (t, $\mathrm{J}=7.1 \mathrm{~Hz}, 3 \mathrm{H}), 1.07(\mathrm{~s}, 9 \mathrm{H})$; MS (ESI+) $\mathrm{m} / \mathrm{z} 593.1(\mathrm{M}+\mathrm{H})^{+}$. Compound 49 was obtained as the second elueting isomer (52 mg, 35\% yield). ${ }^{1} \mathrm{H} \mathrm{NMR}\left(400 \mathrm{MHz}, \mathrm{CDCl}_{3}\right) \delta \mathrm{ppm} 8.22(\mathrm{tt}, \mathrm{J}=2.5,1.2 \mathrm{~Hz}, 1 \mathrm{H})$, 7.72 - $7.61(\mathrm{~m}, 1 \mathrm{H}), 7.52$ - $7.48(\mathrm{~m}, 1 \mathrm{H}), 7.36$ - $7.31(\mathrm{~m}, 1 \mathrm{H}), 7.30$ - $7.23(\mathrm{~m}, 2 \mathrm{H}), 7.19$ - $7.13(\mathrm{~m}, 1 \mathrm{H})$, $5.24(\mathrm{~d}, \mathrm{~J}=5.6 \mathrm{~Hz}, 1 \mathrm{H}), 5.19(\mathrm{~d}, \mathrm{~J}=1.5 \mathrm{~Hz}, 1 \mathrm{H}), 4.34(\mathrm{dd}, \mathrm{J}=24.7,14.0 \mathrm{~Hz}, 1 \mathrm{H}), 4.28-4.12(\mathrm{~m}, 3 \mathrm{H})$, 4.11 - $3.94(\mathrm{~m}, 3 \mathrm{H}), 3.91(\mathrm{~d}, \mathrm{~J}=7.2 \mathrm{~Hz}, 3 \mathrm{H}), 3.83$ - $3.73(\mathrm{~m}, 1 \mathrm{H}), 3.47$ (td, J = 11.1, 2.6 Hz, 1H), 2.70 (s, 1H), 1.99 - $1.60(\mathrm{~m}, 4 \mathrm{H}), 1.56-1.48(\mathrm{~m}, 2 \mathrm{H}), 1.24(\mathrm{t}, \mathrm{J}=7.2 \mathrm{~Hz}, 3 \mathrm{H}), 1.08(\mathrm{~d}, \mathrm{~J}=13.1 \mathrm{~Hz}, 9 \mathrm{H})$; MS $(\mathrm{ESI}+) \mathrm{m} / z 593.2(\mathrm{M}+\mathrm{H})^{+}$.

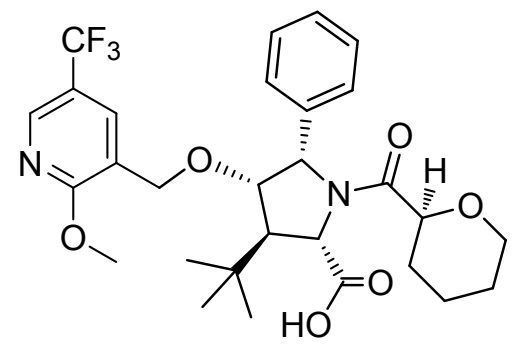

(2S,3R,4S,5S)-3-(tert-butyl)-4-((2-methoxy-5-(trifluoromethyl)pyridin-3-yl)methoxy)-5-phenyl-1((S)-tetrahydro-2H-pyran-2-carbonyl)pyrrolidine-2-carboxylic acid (12)

The mixture of Compound $48(50 \mathrm{mg}, 0.084 \mathrm{mmol})$ in methanol $(1.5 \mathrm{~mL})$ and 6M aqueous $\mathrm{LiOH}(0.5$ $\mathrm{mL}$ ) was stirred at $45^{\circ} \mathrm{C}$ for overnight. The mixture was adjusted to $\mathrm{pH}$ to $1 \sim 2$ by adding $2 \mathrm{M}$ aqueous $\mathrm{HCl}$. The reaction mixture was concentrated, taken up in dichloromethane, and filtered through a syringe filter. The filtrate was purified via chromatography on a $4 \mathrm{~g}$ silica gel cartridge, eluting with methanol in dichloromethane at 0-20\% gradient to provide the title compound (30 mg, 63.0 \% yield). ${ }^{1} \mathrm{H}$ NMR (400 MHz, DMSO- $\left.d_{6}\right) \delta$ ppm $8.37-8.29(\mathrm{~m}, 1 \mathrm{H}), 7.66(\mathrm{~d}, J=7.4 \mathrm{~Hz}, 2 \mathrm{H}), 7.21(\mathrm{~d}, J=7.5 \mathrm{~Hz}, 3 \mathrm{H}), 7.16(\mathrm{~d}, J$ 
$=7.5 \mathrm{~Hz}, 1 \mathrm{H}), 5.42(\mathrm{~s}, 1 \mathrm{H}), 4.55(\mathrm{~s}, 1 \mathrm{H}), 4.33-4.25(\mathrm{~m}, 2 \mathrm{H}), 3.91(\mathrm{~d}, J=13.9 \mathrm{~Hz}, 1 \mathrm{H}), 3.88(\mathrm{~s}, 3 \mathrm{H})$, $3.79(\mathrm{~d}, J=11.3 \mathrm{~Hz}, 1 \mathrm{H}), 3.51(\mathrm{~s}, 1 \mathrm{H}), 2.84(\mathrm{~s}, 2 \mathrm{H}), 1.72-1.26(\mathrm{~m}, 6 \mathrm{H}), 1.00(\mathrm{~s}, 9 \mathrm{H}) ;$ MS (ESI+) $\mathrm{m} / z$ $565.1(\mathrm{M}+\mathrm{H})^{+}$.

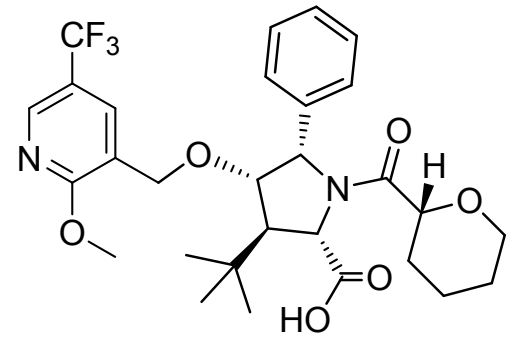

$(2 S, 3 R, 4 S, 5 S)-3-t e r t-b u t y l-4-\{[2-m e t h o x y-5-(t r i f l u o r o m e t h y l) p y r i d i n-3-y l] m e t h o x y\}-1-[(2 R)-o x a n e-2-$ carbonyl]-5-phenylpyrrolidine-2-carboxylic acid (13)

The title compound was prepared from compound 49 according to the procedure described to convery compound 48 to compound 12. ${ }^{1} \mathrm{H}$ NMR (400 MHz, DMSO- $\left.d_{6}\right) \delta$ ppm $8.28(\mathrm{~s}, 1 \mathrm{H}), 7.55(\mathrm{~s}, 2 \mathrm{H}), 7.26$ 7.13 (m, 3H), 7.10 (d, J = 6.9 Hz, 1H), 5.19 (d, J = 6.2 Hz, 1H), 4.81 (s, 1H), 4.364 .20 (m, 2H), 3.88 (s, 4H), $3.79(\mathrm{~d}, \mathrm{~J}=12.0 \mathrm{~Hz}, 1 \mathrm{H}), 2.58(\mathrm{~s}, 1 \mathrm{H}), 1.821 .27(\mathrm{~m}, 7 \mathrm{H}), 1.01(\mathrm{~d}, \mathrm{~J}=3.6 \mathrm{~Hz}, 9 \mathrm{H})$; MS (ESI+) $m / z$ $565.1(\mathrm{M}+\mathrm{H})^{+}$.

Scheme S7. Discovery Synthesis of Compound 14a 
<smiles>CCOC(=O)CN=Cc1ccccc1C(C)C</smiles>

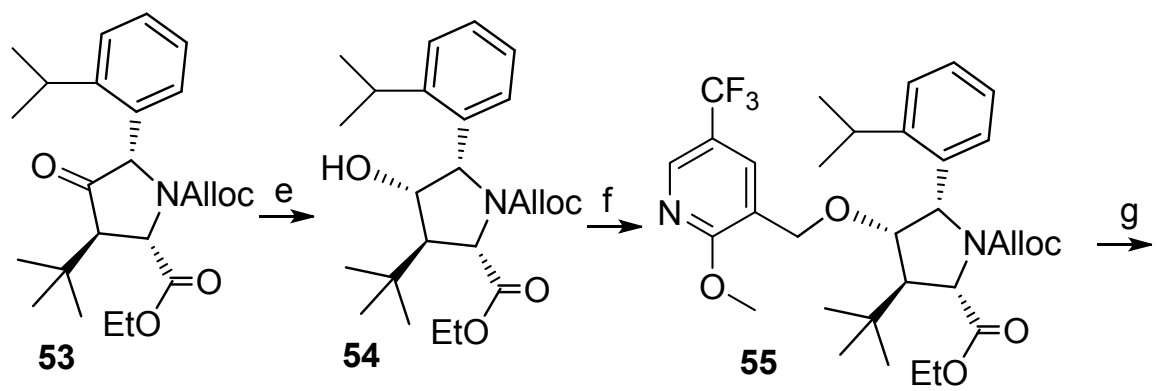<smiles>CCOC(=O)[C@H]1N[C@@H](c2ccccc2C(C)C)[C@H](OCc2cc(C(F)(F)F)cnc2OC)[C@@H]1C(C)(C)C</smiles>

aReagents and conditions:<smiles>CCOC(=O)[C@H]1[C@@H](C(C)(C)C)[C@@H](OCc2cc(C(F)(F)F)cnc2OC)[C@H](c2ccccc2C(C)C)N1C(=O)[C@@H]1CCCCO1</smiles>

(a) $\mathrm{MgSO}_{4}, \quad$ TEA, 2-methylbenzaldehyde, DCM;<smiles>COc1ncc(C(F)(F)F)cc1CO[C@@H]1[C@H](c2ccccc2C(C)C)N(C(=O)[C@H]2CCCCO2)[C@H](C(=O)O)[C@@H]1C(C)(C)C</smiles>

(b) $\quad(2-(\operatorname{Bis}(3,5-$ bis(trifluoromethyl)phenyl)phosphino)-3-((S)-4-isopropyl-4,5-dihydrooxazol-2-yl)cyclopenta-2,4-dien-1-yl)(cyclopenta-2,4-dien1-yl)iron, $\mathrm{Cu}(\mathrm{OTf})_{2}$, KOtBu, (E)-3,3-dimethyl-1-nitrobut-1-ene, THF; (c) allyl chloroformate, toluene; (d) PDC, 6N HCl, Zn dust, (e) $\mathrm{NaBH}_{4}, \mathrm{EtOH}$; (f) $\mathrm{KOtBu}, 3$-(bromomethyl)-2-methoxy-5-(trifluoromethyl)pyridine, DMF; (g) 1,3-Dimethlbarbituric acid, $\mathrm{Pd}\left(\mathrm{PPh}_{3}\right)_{4}$, EtOAc/DCM; (h) Tetrahydropyran-2-carboxylic acid, oxalyl chloride, DMF, DCM; (i) LiOH, MeOH/THF.

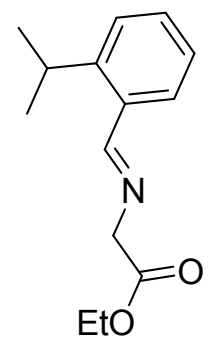

\section{(E)-ethyl 2-((2-isopropylbenzylidene)amino)acetate (50)}

Ethyl 2-aminoacetate hydrochloride $(5.02 \mathrm{~g}, 36.0 \mathrm{mmol})$ and magnesium sulfate $(5.20 \mathrm{~g}, 43.2 \mathrm{mmol})$ were suspended in dichloromethane $(45 \mathrm{~mL})$ and treated with triethylamine $(9.9 \mathrm{~mL}, 71.0 \mathrm{mmol})$. The mixture was stirred for 20 minutes and was treated dropwise with 2-isopropylbenzaldehyde ( $5 \mathrm{~g}, 33.7 \mathrm{mmol}$ ). The 
reaction mixture stirred at room temperature for 3 days. The mixture was filtered (fritted glass funnel), and the filter pad was washed with copious amount of $\mathrm{CH}_{2} \mathrm{Cl}_{2}$. The filtrates were washed twice with water and once with brine, dried over $\mathrm{Na}_{2} \mathrm{SO}_{4}$, filtered, and concentrated in vacuo to yield the title compound, $7.066 \mathrm{~g}$ (90 \% yield). ${ }^{1} \mathrm{H}$ NMR (400 MHz, $\left.\mathrm{CDCl}_{3}\right) \delta \mathrm{ppm} 8.73(\mathrm{~m}, 1 \mathrm{H}), 7.94$ (dd, J = 7.9, 1.5 $\mathrm{Hz}, 1 \mathrm{H}), 7.42(\mathrm{~m}, 1 \mathrm{H}), 7.35(\mathrm{dd}, \mathrm{J}=7.9,1.4 \mathrm{~Hz}, 1 \mathrm{H}), 7.24(\mathrm{~m}, 1 \mathrm{H}), 4.44(\mathrm{~d}, \mathrm{~J}=1.4 \mathrm{~Hz}, 2 \mathrm{H}), 4.26(\mathrm{q}, \mathrm{J}=$ $7.1 \mathrm{~Hz}, 2 \mathrm{H}), 3.53$ (hept, $\mathrm{J}=6.8 \mathrm{~Hz}, 1 \mathrm{H}), 1.34-1.29(\mathrm{~m}, 9 \mathrm{H}) . \mathrm{MS}_{\left(\mathrm{ESI}^{+}\right)} \mathrm{m} / \mathrm{z} 234.1(\mathrm{M}+\mathrm{H})^{+}$.

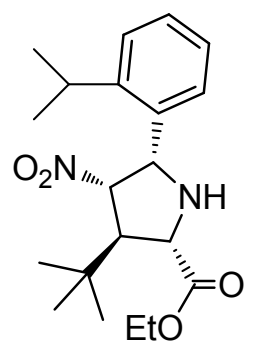

(2S,3R,4S,5S)-ethyl 3-(tert-butyl)-5-(2-isopropylphenyl)-4-nitropyrrolidine-2-carboxylate (51)

Tetrahydrofuran $(30 \mathrm{~mL})$ was sparged with nitrogen for 75 minutes, then it was treated with copper(I) triflate dimer, benzene complex (0.033 g, $0.065 \mathrm{mmol})$ and (2-(bis(3,5bis(trifluoromethyl)phenyl)phosphino)-3-((S)-4-isopropyl-4,5-dihydrooxazol-2-yl)cyclopenta-2,4-dien-1yl)(cyclopenta-2,4-dien-1-yl)iron (0.098 g, $0.129 \mathrm{mmol})$. The mixture was stirred at room temperature for 1 hour. The mixture was cooled to $<5^{\circ} \mathrm{C}$ and treated dropwise with a solution of compound $\mathbf{5 0}$ (4.15 $\mathrm{g}, 17.80 \mathrm{mmol})$ in $8 \mathrm{~mL}$ tetrahydrofuran, followed by dropwise addition of potassium 2-methylpropan-2olate ( $1 \mathrm{M}$ in tetrahydrofuran; $0.1 \mathrm{~mL}, 0.100 \mathrm{mmol})$, keeping the temperature $<5{ }^{\circ} \mathrm{C}$. Neat $(E)-3,3-$ dimethyl-1-nitrobut-1-ene (2.09 g, $16.18 \mathrm{mmol})$ was then added dropwise over about 10 minutes to keep the temperature $<10^{\circ} \mathrm{C}$. After completion of the addition, the reaction continued to stir in the ice bath for 25 minutes. The reaction mixture was then quenched with $25 \mathrm{~mL}$ of saturated aqueous $\mathrm{NH}_{4} \mathrm{Cl}$ solution and warmed up to room temperature. The mixture was diluted with methyl tert-butyl ether and washed twice with saturated aqueous $\mathrm{NH}_{4} \mathrm{Cl}$ solution and once with brine. The organic layer was dried over $\mathrm{Na}_{2} \mathrm{SO}_{4}$, filtered, and concentrated in vacuo. Silica gel chromatography, eluting with 10 to $50 \%$ ethyl acetate-heptanes, afforded the title compound, $1.902 \mathrm{~g}$, (32\% yield). ${ }^{1} \mathrm{H}$ NMR (400 MHz, DMSO$\left.d_{6}\right) \delta$ ppm 7.42 - $7.34(\mathrm{~m}, 1 \mathrm{H}), 7.30-7.17(\mathrm{~m}, 2 \mathrm{H}), 7.08(\mathrm{ddd}, \mathrm{J}=7.5,6.9,1.7 \mathrm{~Hz}, 1 \mathrm{H}), 5.08(\mathrm{dd}, \mathrm{J}=6.9$, $3.6 \mathrm{~Hz}, 1 \mathrm{H}), 4.77(\mathrm{~d}, \mathrm{~J}=6.9 \mathrm{~Hz}, 1 \mathrm{H}), 4.23$ (q, J = 7.1 Hz, 2H), 3.77 (d, J = 7.6 Hz, 1H), 3.25 (br s, 1H), $3.21-3.06(\mathrm{~m}, 2 \mathrm{H}), 1.33-1.14(\mathrm{~m}, 9 \mathrm{H}), 0.98(\mathrm{~s}, 9 \mathrm{H})$. $\mathrm{MS}\left(\mathrm{ESI}^{+}\right) \mathrm{m} / z 363.1(\mathrm{M}+\mathrm{H})^{+}$. 


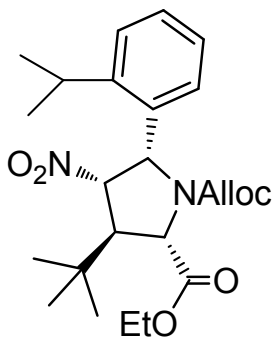

(2S,3R,4S,5S)-1-allyl 2-ethyl 3-(tert-butyl)-5-(2-isopropylphenyl)-4-nitropyrrolidine-1,2dicarboxylate (52)

Compound 51 (12.3 g, $33.9 \mathrm{mmol})$ was dissolved in toluene $(120 \mathrm{~mL})$ and saturated aqueous sodium bicarbonate $(120 \mathrm{~mL})$ was added. Allyl chloroformate $(3.98 \mathrm{~mL}, 37.3 \mathrm{mmol})$ was added, and the reaction mixture was stirred at ambient temperature for 20 minutes, at which point LC/MS indicated complete conversion of the starting material. The reaction was diluted with methyl tert-butyl ether $(70 \mathrm{~mL})$ and the layers were separated. The organic layer was washed with $1 \mathrm{M}$ aqueous $\mathrm{HCl}(2 \times 30 \mathrm{~mL}), 1 \mathrm{M}$ aqueous $\mathrm{NaOH}(2 \times 30 \mathrm{~mL})$ and brine $(30 \mathrm{~mL})$. The organic layer was dried over sodium sulfate, filtered, concentrated, and purified via flash chromatography, eluting with 0:100 to 30:70 methyl tert-butyl ether:heptanes over 30 minutes on a $330 \mathrm{~g}$ silica gel column to provide $12.3 \mathrm{~g}$ of the title compound. ${ }^{1} \mathrm{H}$ NMR (400 MHz, DMSO- $\left.d_{6}, 120^{\circ} \mathrm{C}\right) \delta \mathrm{ppm} 7.86(\mathrm{dd}, J=8.0,1.2 \mathrm{~Hz}, 1 \mathrm{H}), 7.32-7.14(\mathrm{~m}, 2 \mathrm{H}), 7.05$ (ddd, $J=8.4,6.5,2.1 \mathrm{~Hz}, 1 \mathrm{H}), 5.72(\mathrm{~d}, J=9.0 \mathrm{~Hz}, 1 \mathrm{H}), 5.65$ (ddd, $J=17.2,10.7,5.3 \mathrm{~Hz}, 1 \mathrm{H}), 5.53(\mathrm{dd}, J$ $=8.9,3.6 \mathrm{~Hz}, 1 \mathrm{H}), 5.08-4.93(\mathrm{~m}, 2 \mathrm{H}), 4.55(\mathrm{~d}, J=4.3 \mathrm{~Hz}, 1 \mathrm{H}), 4.41(\mathrm{~d}, J=5.2 \mathrm{~Hz}, 2 \mathrm{H}), 4.27(\mathrm{qd}, J=$ 7.0, $0.9 \mathrm{~Hz}, 2 \mathrm{H}), 3.24$ (p, $J=6.7 \mathrm{~Hz}, 1 \mathrm{H}), 3.14-3.05(\mathrm{~m}, 1 \mathrm{H}), 1.31$ (t, $J=7.1 \mathrm{~Hz}, 3 \mathrm{H}), 1.27(\mathrm{~d}, J=6.6$ $\mathrm{Hz}, 3 \mathrm{H}), 1.19(\mathrm{~d}, J=6.7 \mathrm{~Hz}, 3 \mathrm{H}), 1.03(\mathrm{~s}, 9 \mathrm{H}) ; \mathrm{MS}(\mathrm{ESI}+) \mathrm{m} / \mathrm{z} 447.3(\mathrm{M}+\mathrm{H})^{+}$.

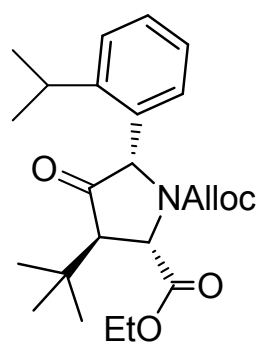

(2S,3R,5S)-1-allyl 2-ethyl 3-(tert-butyl)-5-(2-isopropylphenyl)-4-oxopyrrolidine-1,2-dicarboxylate (53)

Compound 52 (11.2 g, $25.08 \mathrm{mmol})$ was dissolved in ethanol $(226 \mathrm{~mL})$ and the solution was heated to $75^{\circ} \mathrm{C}$. A separate solution of $\mathrm{CrCl}_{2}$ was prepared by dissolving pyridinium dichromate (23.59 g, $62.7 \mathrm{mmol})$ in aqueous hydrochloric acid, (6M, $452 \mathrm{~mL})$ and adding $\mathrm{Zn}(44.3 \mathrm{~g}, 667$ mmol) in portions while cooling in an ice bath, maintaining an internal temperature below $35{ }^{\circ} \mathrm{C}$. 
The resulting suspension was stirred at ambient temperature for 30 minutes after removing the flask from the ice bath, leaving a blue solution of $\mathrm{CrCl}_{2}$, which was added via cannula over 20 minutes to the reaction flask containing the solution of the starting material in ethanol at $75{ }^{\circ} \mathrm{C}$. Heating was continued at $85^{\circ} \mathrm{C}$ for 36 hours. The flask was cooled to ambient temperature, and the organic material was extracted with dichloromethane $(3 \times 200 \mathrm{~mL})$. The combined extracts were dried over sodium sulfate, filtered, and concentrated. The material was redissolved in an anhydrous solution of $\mathrm{HCl}$ in ethanol that was prepared by the addition of acetyl chloride (8.9 $\mathrm{mL}, 125 \mathrm{mmol}$ ) to $100 \mathrm{~mL}$ of ethanol via syringe while cooling in an ice-water bath. The resulting solution was heated to $45^{\circ} \mathrm{C}$ for 1 hour, at which point complete re-esterification had occurred. The reaction mixture was concentrated and the crude material was partitioned between saturated aqueous sodium bicarbonate and methyl tert-butyl ether (300 mL each). The organic extracts were dried over sodium sulfate, filtered, and concentrated to provide $(2 S, 3 R, 5 S)$-1-allyl 2-ethyl 3-(tert-butyl)-5-(2-isopropylphenyl)-4-oxopyrrolidine-1,2-dicarboxylate (10 g) as an inseparable mixture with unreacted oxime. The mixture was used in the next step without additional purification

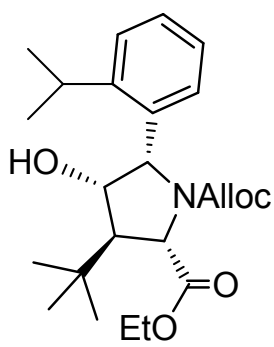

(2S,3R,4S,5S)-1-allyl 2-ethyl 3-(tert-butyl)-4-hydroxy-5-(2-isopropylphenyl)pyrrolidine-1,2dicarboxylate (54)

Compound 53 (10 g, $24.07 \mathrm{mmol})$ was dissolved in $100 \mathrm{~mL}$ of ethanol and sodium borohydride $(0.910 \mathrm{~g}$, $24.07 \mathrm{mmol}$ ) was added in one portion after cooling to $-5^{\circ} \mathrm{C}$. The reaction was complete in 10 minutes at this temperature, as indicated by LC/MS. Acetone $(10 \mathrm{~mL})$ was added to quench the excess borohydride, and the reaction mixture was stirred at the same temperature for 15 minutes. The flask was warmed to ambient temperature, concentrated in vacuo, and partitioned between methyl tert-butyl ether and saturated sodium bicarbonate (100 $\mathrm{mL}$ each). The organic extracts were concentrated in vacuo to provide crude material, which was purified via flash chromatography, eluting with 0:100 to 30:70 methyl tert-butyl ether:heptanes over 40 minutes on a $220 \mathrm{~g}$ silica gel column to provide $5.5 \mathrm{~g}(41 \%)$ of the desired diastereomer (2S,3R,4S,5S)-1-allyl 2-ethyl 3-(tert-butyl)-4-hydroxy-5-(2-isopropylphenyl)pyrrolidine-1,2- 
dicarboxylate as an inseparable mixture with the oxime from the previous step. ${ }^{1} \mathrm{H}$ NMR $(400 \mathrm{MHz}$, DMSO- $\left.d_{6}, 120^{\circ} \mathrm{C}\right) \delta \mathrm{ppm} 7.90(\mathrm{dd}, J=7.8,1.5 \mathrm{~Hz}, 1 \mathrm{H}), 7.20(\mathrm{ddd}, J=7.7,4.4,1.5 \mathrm{~Hz}, 1 \mathrm{H}), 7.13(\mathrm{tdd}, J$ $=7.6,2.8,1.5 \mathrm{~Hz}, 1 \mathrm{H}), 7.05(\mathrm{ddd}, J=8.9,7.2,1.5 \mathrm{~Hz}, 1 \mathrm{H}), 5.70-5.59(\mathrm{~m}, 1 \mathrm{H}), 5.22(\mathrm{~d}, J=6.6 \mathrm{~Hz}, 1 \mathrm{H})$, $5.04-4.84(\mathrm{~m}, 2 \mathrm{H}), 4.35(\mathrm{~m}, 3 \mathrm{H}), 4.30(\mathrm{~d}, J=4.6 \mathrm{~Hz}, 1 \mathrm{H}), 4.24-4.14(\mathrm{~m}, 2 \mathrm{H}), 3.75$ (br s, 1H), 3.19 (dt, $J=13.6,6.9 \mathrm{~Hz}, 1 \mathrm{H}), 2.37-2.25(\mathrm{~m}, 1 \mathrm{H}), 1.26(\mathrm{~d}, J=5.7 \mathrm{~Hz}, 3 \mathrm{H}), 1.24(\mathrm{t},, J=4 \mathrm{~Hz}, 3 \mathrm{H}), 1.20(\mathrm{~d}, J=$ $5.7 \mathrm{~Hz}, 3 \mathrm{H}), 1.00(\mathrm{~s}, 9 \mathrm{H})$; MS (ESI+) $m / z 418.1(\mathrm{M}+\mathrm{H})^{+}$.

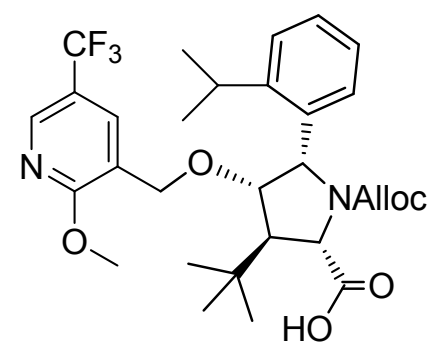

(2S,3R,4S,5S)-1-allyl 2-ethyl 3-(tert-butyl)-5-(2-isopropylphenyl)-4-((2-methoxy-5(trifluoromethyl)pyridin-3-yl)methoxy)pyrrolidine-1,2-dicarboxylate (55)

Compound 54 (1.75 g, $3.14 \mathrm{mmol})$ was dissolved in $1.2 \mathrm{~mL}$ of dry dimethylformamide. After cooling to $0{ }^{\circ} \mathrm{C}$ in an ice bath, potassium tert-butoxide $(1 \mathrm{M}$ in tetrahydrofuran, $4.6 \mathrm{~mL}, 4.6 \mathrm{mmol})$ solution was added dropwise, followed immediately by addition of 3-(bromomethyl)-2-methoxy-5(trifluoromethyl)pyridine $(1.36 \mathrm{~g}, 5.0 \mathrm{mmol})$ dropwise via syringe. LC/MS after 10 minutes showed complete conversion of the starting alcohol. The reaction was quenched with saturated aqueous ammonium chloride $(10 \mathrm{~mL})$ and extracted with methyl tert-butyl ether $(3 \times 20 \mathrm{~mL})$. The combined organic extracts were concentrated in vacuo and purified via flash chromatography, eluting on a $120 \mathrm{~g}$ silica gel column with 0:100 to 20:80 methyl tert-butyl ether:heptanes over 20 minutes to provide $955 \mathrm{mg}$ $(50 \%)$ of the title compound. ${ }^{1} \mathrm{H}$ NMR $\left(400 \mathrm{MHz}, \mathrm{DMSO}-d_{6}, 120^{\circ} \mathrm{C}\right) \delta \mathrm{ppm} 8.33-8.21(\mathrm{~m}, 1 \mathrm{H}), 7.92$ $(\mathrm{dd}, J=7.9,1.4 \mathrm{~Hz}, 1 \mathrm{H}), 7.17(\mathrm{dt}, J=5.7,1.5 \mathrm{~Hz}, 2 \mathrm{H}), 7.10(\mathrm{td}, J=7.5,1.4 \mathrm{~Hz}, 1 \mathrm{H}), 7.01-6.93$ (m, $1 \mathrm{H}), 5.66(\mathrm{ddt}, J=16.2,10.4,5.1 \mathrm{~Hz}, 1 \mathrm{H}), 5.33(\mathrm{~d}, J=5.7 \mathrm{~Hz}, 1 \mathrm{H}), 5.04-4.87(\mathrm{~m}, 2 \mathrm{H}), 4.50(\mathrm{~d}, J=2.2$ $\mathrm{Hz}, 1 \mathrm{H}), 4.39$ (d, $J=5.2 \mathrm{~Hz}, 2 \mathrm{H}), 4.29-4.22$ (m, 2H), 4.13 (qd, $J=7.1,1.2 \mathrm{~Hz}, 2 \mathrm{H}), 3.86$ (s, 3H), 3.78 $(\mathrm{d}, J=13.8 \mathrm{~Hz}, 1 \mathrm{H}), 3.24$ (p, $J=6.8 \mathrm{~Hz}, 1 \mathrm{H}), 2.52$ (t, $J=1.6 \mathrm{~Hz}, 1 \mathrm{H}), 1.23$ (d, $J=6.7 \mathrm{~Hz}, 3 \mathrm{H}), 1.19(\mathrm{~d}, J$ $=7.0 \mathrm{~Hz}, 3 \mathrm{H}), 1.15(\mathrm{t}, J=8 \mathrm{~Hz}, 3 \mathrm{H}), 1.05(\mathrm{~s}, 9 \mathrm{H})$; MS (ESI+) $m / z 607.0(\mathrm{M}+\mathrm{H})^{+}$. 


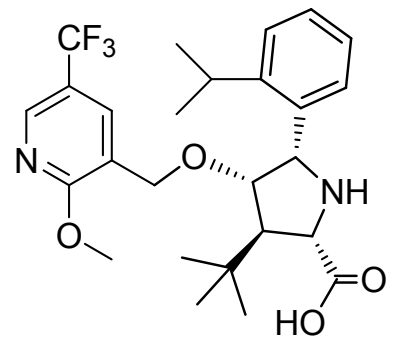

(2S,3R,4S,5S)-ethyl 3-(tert-butyl)-5-(2-isopropylphenyl)-4-((2-methoxy-5-(trifluoromethyl)pyridin3-yl)methoxy)pyrrolidine-2-carboxylate (56)

Compound 55 (850 mg, $1.401 \mathrm{mmol}$ ) and 1,3-dimethylpyrimidine-2,4,6(1H,3H,5H)-trione (438 mg, 2.80 mmol) were dissolved in $14 \mathrm{~mL}$ of a 1:1 mixture of dichloromethane and ethyl acetate that had been degassed for 5 minutes. Palladium tetrakis(triphenylphosphine)palladium (16.19 mg, $0.014 \mathrm{mmol}$ ) was added, and the reaction mixture was stirred at ambient temperature for 15 minutes, at which point the reaction was complete as indicated by LC/MS. The reaction mixture was diluted with methyl tert-butyl ether $(50 \mathrm{~mL})$ and stirred with $10 \mathrm{~mL}$ of $10 \%$ aqueous sodium carbonate solution for 10 minutes. The organic layer was washed with brine and concentrated in vacuo to provide crude material. The crude material was purified via flash chromatography, eluting with 0:100 to 30:70 methyl tert-butyl ether:heptanes over 20 minutes on a $40 \mathrm{~g}$ silica gel column to provide $623 \mathrm{mg}(85 \%)$ of $(2 S, 3 R, 4 S, 5 S)$ ethyl 3-(tert-butyl)-5-(2-isopropylphenyl)-4-((2-methoxy-5-(trifluoromethyl)pyridin-3yl)methoxy)pyrrolidine-2-carboxylate. ${ }^{1} \mathrm{H}$ NMR $\left(400 \mathrm{MHz}\right.$, DMSO- $\left.d_{6}, 120{ }^{\circ} \mathrm{C}\right) \delta \mathrm{ppm} 8.34-8.26(\mathrm{~m}$, 1H), 7.63 (dd, $J=7.9,1.4 \mathrm{~Hz}, 1 \mathrm{H}), 7.39$ (d, $J=2.4 \mathrm{~Hz}, 1 \mathrm{H}), 7.22$ (dd, $J=7.8,1.5 \mathrm{~Hz}, 1 \mathrm{H}), 7.14$ (td, $J=$ 7.5, $1.4 \mathrm{~Hz}, 1 \mathrm{H}), 7.02(\mathrm{td}, J=7.6,1.4 \mathrm{~Hz}, 1 \mathrm{H}), 4.42(\mathrm{~d}, J=4.5 \mathrm{~Hz}, 1 \mathrm{H}), 4.22(\mathrm{~d}, J=13.6 \mathrm{~Hz}, 1 \mathrm{H}), 4.15$ (q, $J=8 \mathrm{~Hz}, 2 \mathrm{H}), 3.96(\mathrm{dd}, J=4.6,1.8 \mathrm{~Hz}, 1 \mathrm{H}), 3.85(\mathrm{~s}, 3 \mathrm{H}), 3.77(\mathrm{~d}, J=13.7 \mathrm{~Hz}, 1 \mathrm{H}), 3.65(\mathrm{~d}, J=6.5$ $\mathrm{Hz}, 1 \mathrm{H}), 3.24$ (p, $J=6.8 \mathrm{~Hz}, 1 \mathrm{H}), 2.42(\mathrm{dd}, J=6.5,1.8 \mathrm{~Hz}, 1 \mathrm{H}), 1.22(\mathrm{t}, J=8 \mathrm{~Hz}, 3 \mathrm{H}), 1.21$ (br s, 3H), 1.19 (br s, 3H) 1.00 (s, 9H); MS (ESI+) $m / z 523.1(\mathrm{M}+\mathrm{H})^{+}$.

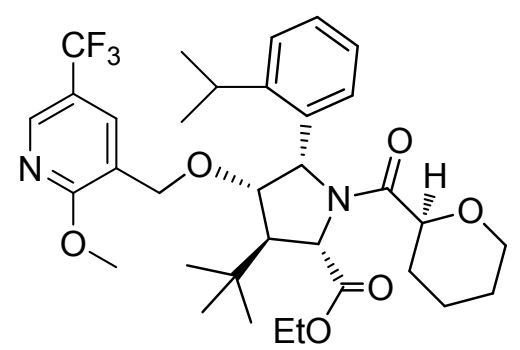

(2S,3R,4S,5S)-ethyl 3-(tert-butyl)-5-(2-isopropylphenyl)-4-((2-methoxy-5-(trifluoromethyl)pyridin3-yl)methoxy)-1-((S)-tetrahydro-2H-pyran-2-carbonyl)pyrrolidine-2-carboxylate (57)

Compound 56 (370 mg, $0.71 \mathrm{mmol})$ was dissolved in dichloromethane $(7.1 \mathrm{~mL})$ and triethylamine $(0.30$ $\mathrm{mL}, 2.1 \mathrm{mmol}$ ) was added. After cooling in an ice bath to $<10^{\circ} \mathrm{C}$, a solution of freshly-prepared 
tetrahydro-2H-pyran-2-carbonyl chloride (158 mg, $1.062 \mathrm{mmol}$ ) was added dropwise, and the reaction was complete as soon as the addition was done, as indicated by LC/MS. The reaction mixture was diluted with methyl tert-butyl ether $(10 \mathrm{~mL})$ and $1 \mathrm{M}$ aqueous $\mathrm{HCl}(10 \mathrm{~mL})$ was added. The layers were separated, and the organic layer was washed with $1 \mathrm{M}$ aqueous $\mathrm{HCl}(2 \times 5 \mathrm{~mL})$ and brine $(10 \mathrm{~mL})$, dried over sodium sulfate, filtered, and concentrated in vacuo to provide a crude residue. The crude material was purified via flash chromatography, eluting with 0:100 to 20:80 methyl tert-butyl ether:heptanes over 5 minutes then isocratic 20:80 methyl tert-butyl ether:heptanes for 40 minutes on an $80 \mathrm{~g}$ silica gel column to provide Compound 57 (144 mg) as the first-eluting diastereomer and (2S,3R,4S,5S)-ethyl 3(tert-butyl)-5-(2-isopropylphenyl)-4-((2-methoxy-5-(trifluoromethyl)pyridin-3-yl)methoxy)-1-((R)tetrahydro-2H-pyran-2-carbonyl)pyrrolidine-2-carboxylate (120 mg, $27 \%$ ) as the second-eluting diastereomer. Compound 57: ${ }^{1} \mathrm{H}$ NMR $\left(400 \mathrm{MHz}\right.$, DMSO- $\left.d_{6}, 120{ }^{\circ} \mathrm{C}\right) \delta \mathrm{ppm} 8.33-8.19(\mathrm{~m}, 1 \mathrm{H}), 8.00$ $(\mathrm{dd}, J=7.9,1.5 \mathrm{~Hz}, 1 \mathrm{H}), 7.24(\mathrm{~d}, J=7.6 \mathrm{~Hz}, 1 \mathrm{H}), 7.15(\mathrm{~s}, 2 \mathrm{H}), 7.03$ (d, $J=7.9 \mathrm{~Hz}, 1 \mathrm{H}), 5.61(\mathrm{~d}, J=0.8$ $\mathrm{Hz}, 1 \mathrm{H}), 4.74(\mathrm{~d}, J=1.8 \mathrm{~Hz}, 1 \mathrm{H}), 4.29(\mathrm{~d}, J=13.8 \mathrm{~Hz}, 1 \mathrm{H}), 4.24(\mathrm{~d}, J=5.7 \mathrm{~Hz}, 1 \mathrm{H}), 4.09$ (qd, $J=7.1$, $2.9 \mathrm{~Hz}, 2 \mathrm{H}), 3.86(\mathrm{~s}, 3 \mathrm{H}), 3.80(\mathrm{~d}, J=13.9 \mathrm{~Hz}, 1 \mathrm{H}), 3.73(\mathrm{~s}, 1 \mathrm{H}), 3.24(\mathrm{p}, J=6.8 \mathrm{~Hz}, 1 \mathrm{H}), 3.18-2.93(\mathrm{~m}$, $1 \mathrm{H}), 2.45(\mathrm{~d}, J=1.7 \mathrm{~Hz}, 2 \mathrm{H}), 1.74-1.61(\mathrm{~m}, 1 \mathrm{H}), 1.54(\mathrm{t}, J=10.7 \mathrm{~Hz}, 2 \mathrm{H}), 1.37(\mathrm{~s}, 3 \mathrm{H}), 1.30(\mathrm{~d}, J=6.7$ $\mathrm{Hz}, 3 \mathrm{H}), 1.16(\mathrm{~d}, J=6.6 \mathrm{~Hz}, 3 \mathrm{H}), 1.13(\mathrm{t}, J=7.1 \mathrm{~Hz}, 3 \mathrm{H}), 1.05(\mathrm{~s}, 9 \mathrm{H})$; MS (ESI+) $m / z 635.1(\mathrm{M}+\mathrm{H})^{+}$.

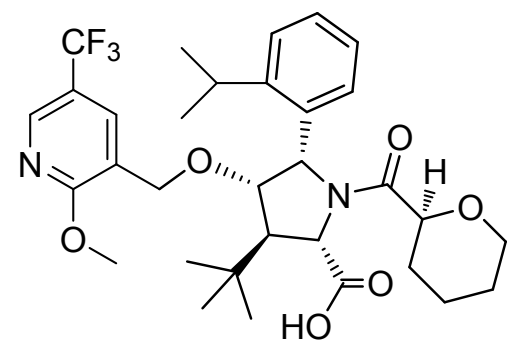

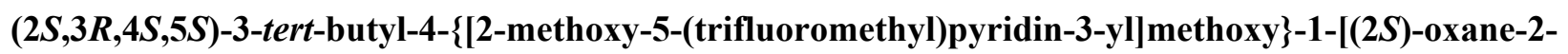
carbonyl]-5-[2-(propan-2-yl)phenyl]pyrrolidine-2-carboxylic acid (14)

Compound 57 (140 mg, $0.22 \mathrm{mmol}$ ) was dissolved in a mixture of $0.9 \mathrm{~mL}$ of tetrahydrofuran, $0.9 \mathrm{~mL}$ of water, and $0.45 \mathrm{~mL}$ of methanol. Lithium hydroxide hydrate (93 $\mathrm{mg}, 2.21 \mathrm{mmol}$ ) was added, and the reaction mixture was heated to $50{ }^{\circ} \mathrm{C}$ for 16 hours. The reaction mixture was cooled to room temperature, diluted with dichloromethane $(10 \mathrm{~mL})$, acidified with $1 \mathrm{M}$ aqueous $\mathrm{HCl}$ to $\mathrm{pH}=3$, and extracted with $3 \mathrm{x}$ $5 \mathrm{~mL}$ dichloromethane. The organic extracts were combined, dried over sodium sulfate, filtered, and concentrated in vacuo to provide Compound 14. X-ray confirmation of the absolute stereochemistry confirmed the identity of the title compound. ${ }^{1} \mathrm{H}$ NMR $\left(400 \mathrm{MHz}, \mathrm{DMSO}-d_{6}, 120^{\circ} \mathrm{C}\right) \delta \mathrm{ppm} 8.33-8.18$ (m, 1H), $8.06(\mathrm{dd}, J=8.0,1.3 \mathrm{~Hz}, 1 \mathrm{H}), 7.32-6.93(\mathrm{~m}, 4 \mathrm{H}), 5.63(\mathrm{~s}, 1 \mathrm{H}), 4.71(\mathrm{~d}, J=1.7 \mathrm{~Hz}, 1 \mathrm{H}), 4.33$ $(\mathrm{d}, J=14.0 \mathrm{~Hz}, 1 \mathrm{H}), 4.24(\mathrm{~d}, J=5.8 \mathrm{~Hz}, 1 \mathrm{H}), 3.87(\mathrm{~s}, 3 \mathrm{H}), 3.84(\mathrm{~d}, J=8.0 \mathrm{~Hz}, 1 \mathrm{H}) 3.75(\mathrm{~d}, J=11.2 \mathrm{~Hz}$, 
1H), 3.24 (p, $J=6.8 \mathrm{~Hz}, 1 \mathrm{H}), 3.20-2.80$ (br s, 2H), 2.52 (d, $J=1.7 \mathrm{~Hz}, 1 \mathrm{H}), 1.73-1.22(\mathrm{~m}, 6 \mathrm{H}), 1.30$ (d, $J=6.7 \mathrm{~Hz}, 3 \mathrm{H}), 1.13(\mathrm{~d}, J=6.7 \mathrm{~Hz}, 3 \mathrm{H}), 1.04(\mathrm{~s}, 9 \mathrm{H})$; MS (ESI+) $m / z 605.3(\mathrm{M}-\mathrm{H})^{-}$.

\section{Scheme S8. Discovery Synthesis of Compound 15a}
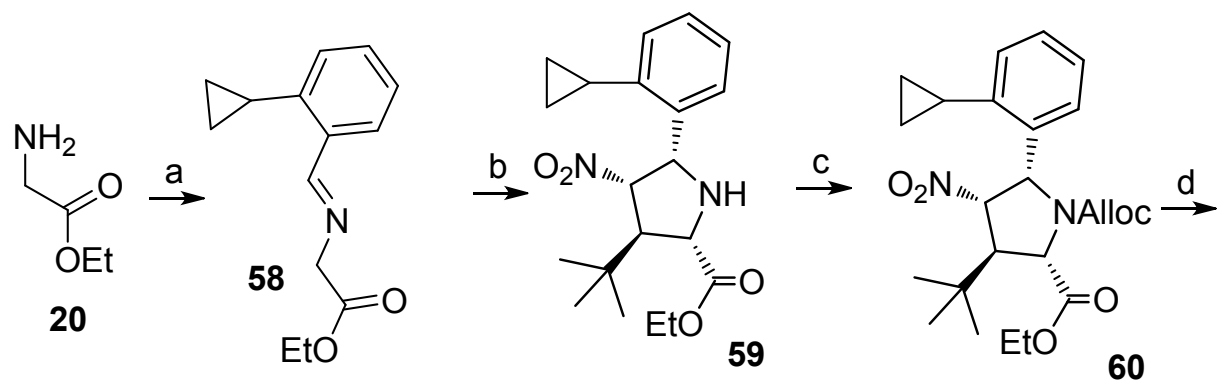<smiles>CCOC(=O)[C@@H]1[C@@H](C(C)(C)C)C(=O)[C@@H](c2ccccc2C2CC2)N1C(C)(C)C</smiles>

61<smiles>CCOC(=O)[C@@H]1[C@@H](C(C)(C)C)[C@@H](O)[C@H](c2ccccc2C2CC2)N1C(C)C</smiles>

62<smiles>CCOC(=O)[C@@H]1[C@@H](C(C)(C)C)[C@@H](OCc2cc(C(F)(F)F)cnc2OC)[C@H](c2ccccc2C2CC2)[C@H]1C(C)(C)C</smiles><smiles>CCOC(=O)[C@H]1N[C@@H](c2ccccc2C2CC2)[C@H](OCc2cc(C(F)(F)F)cnc2OC)[C@@H]1C(C)(C)C</smiles>

aReagents and conditions:<smiles>CCOC(=O)[C@H]1[C@@H](C(C)(C)C)[C@@H](OCc2cc(C(F)(F)F)cnc2OC)[C@H](c2ccccc2C2CC2)N1C(=O)[C@H]1CCCCO1</smiles>
65<smiles>COc1ncc(C(F)(F)F)cc1CO[C@@H]1[C@H](c2ccccc2C2CC2)N(C(=O)[C@H]2CCCCO2)[C@H](C(=O)O)[C@H]1C(C)(C)C</smiles>

(a) $\quad \mathrm{MgSO}_{4}, \quad$ TEA, 2-methylbenzaldehyde, DCM; (b) (2-(Bis(3,5bis(trifluoromethyl)phenyl)phosphino)-3-((S)-4-isopropyl-4,5-dihydrooxazol-2-yl)cyclopenta-2,4-dien-1-yl)(cyclopenta-2,4-dien1-yl)iron, $\mathrm{Cu}(\mathrm{OTf})_{2}$, KOtBu, (E)-3,3-dimethyl-1-nitrobut-1-ene, THF; (c) allyl chloroformate, toluene; (d) PDC, 6N HCl, Zn dust, (e) $\mathrm{NaBH}_{4}, \mathrm{EtOH}$; (f) KOtBu, 3-(bromomethyl)-2-methoxy-5-(trifluoromethyl)pyridine, DMF; (g) 1,3-Dimethlbarbituric acid, $\mathrm{Pd}\left(\mathrm{PPh}_{3}\right)_{4}$, EtOAc/DCM; (h) Tetrahydropyran-2-carboxylic acid, oxalyl chloride, DMF, DCM; (i) LiOH, MeOH/THF. 


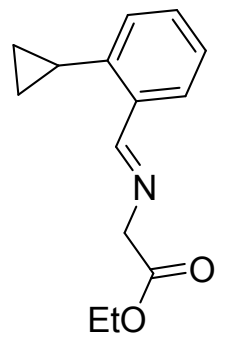

\section{(E)-ethyl 2-((2-cyclopropylbenzylidene)amino)acetate (58)}

Ethyl 2-aminoacetate hydrochloride $(4.50 \mathrm{~g}, 32.2 \mathrm{mmol})$ and magnesium sulfate $(6.21 \mathrm{~g}, 51.6 \mathrm{mmol})$ were suspended in dichloromethane $(43.0 \mathrm{~mL})$ and the suspension was treated with triethylamine $(4.49 \mathrm{~mL}$, $32.2 \mathrm{mmol})$. After 1 hour, 2-cyclopropylbenzaldehyde (3.77 g, $25.8 \mathrm{mmol})$ in $5 \mathrm{~mL}$ of dichloromethane was added and the reaction was stirred at room temperature for 16 hours. The solid material was removed via filtration and the filtrate was washed with water and brine, dried over sodium sulfate, filtered, and concentrated to provide $(E)$-ethyl 2-((2-cyclopropylbenzylidene)amino)acetate (5.68 g, $24.56 \mathrm{mmol}, 95 \%$ yield), which was used in the next step without further purification. ${ }^{1} \mathrm{H}$ NMR (400 MHz, DMSO- $\left.d_{6}\right) \delta$ ppm $8.92(\mathrm{~d}, J=1.5 \mathrm{~Hz}, 1 \mathrm{H}), 7.81(\mathrm{dd}, J=7.8,1.5 \mathrm{~Hz}, 1 \mathrm{H}), 7.35(\mathrm{td}, J=7.6,1.5 \mathrm{~Hz}, 1 \mathrm{H}), 7.22(\mathrm{td}, J=$ 7.6, 1.2 Hz, 1H), 7.05 (dd, $J=7.8,1.2 \mathrm{~Hz}, 1 \mathrm{H}), 4.45$ (d, $J=1.3 \mathrm{~Hz}, 2 \mathrm{H}), 4.13$ (q, $J=7.1 \mathrm{~Hz}, 2 \mathrm{H}), 2.33$ $(\mathrm{tt}, J=8.5,5.3 \mathrm{~Hz}, 1 \mathrm{H}), 1.21(\mathrm{t}, J=7.1 \mathrm{~Hz}, 3 \mathrm{H}), 1.03-0.90(\mathrm{~m}, 2 \mathrm{H}), 0.75-0.63(\mathrm{~m}, 2 \mathrm{H}) . \mathrm{MS}\left(\mathrm{ESI}^{+}\right)$ $m / z 232.1(\mathrm{M}+\mathrm{H})^{+}$.

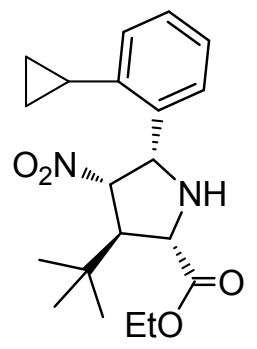

(2S,3R,4S,5S)-ethyl 3-(tert-butyl)-5-(2-cyclopropylphenyl)-4-nitropyrrolidine-2-carboxylate (59) (2-(Bis(3,5-bis(trifluoromethyl)phenyl)phosphino)-3-((S)-4-isopropyl-4,5-dihydrooxazol-2-yl)cyclopenta2,4-dien-1-yl)(cyclopenta-2,4-dien-1-yl)iron (0.241 g, $0.319 \mathrm{mmol})$ and copper (I) triflate dimer, benzene complex $(0.062 \mathrm{~g}, 0.123 \mathrm{mmol})$ were dissolved in tetrahydrofuran $(63.0 \mathrm{~mL})$ that had been sparged with an nitrogen stream for 2 hours. The resulting mixture was stirred for 1.5 hours at room temperature, and (E)-ethyl 2-((2-cyclopropylbenzylidene)amino)acetate, $(\mathbf{5 8}, 5.68 \mathrm{~g}, 24.56 \mathrm{mmol})$ in tetrahydrofuran ( 8 $\mathrm{mL})$ was added after cooling to $<5{ }^{\circ} \mathrm{C}$ in an ice-water bath. Potassium 2-methylpropan-2-olate $(0.246$ $\mathrm{mL}, 0.246 \mathrm{mmol}$ ) was added drop wise, followed by addition of $(E)$-3,3-dimethyl-1-nitrobut-1-ene (3.17 $\mathrm{g}, 24.56 \mathrm{mmol}$ ) neat over 25 minutes, maintaining an internal temperature $<10{ }^{\circ} \mathrm{C}$. After the addition was complete, the reaction was stirred for 15 minutes at the same temperature, diluted with methyl tert-butyl 
ether $(100 \mathrm{~mL})$ and stirred with $75 \mathrm{~mL}$ of saturated ammonium chloride at room temperature for 15 minutes. The organic layer was separated, washed with saturated sodium bicarbonate and brine, dried over sodium sulfate, and filtered. The filtrate was concentrated and purified by flash chromatography ( 0 to $30 \%$ ethyl acetate in heptane) to provide $(2 S, 3 R, 4 S, 5 S)$-ethyl 3-(tert-butyl)-5-(2-cyclopropylphenyl)-4nitropyrrolidine-2-carboxylate (6.85 g, $19.00 \mathrm{mmol}, 77 \%$ yield) . ee > 97\%. ${ }^{1} \mathrm{H}$ NMR $(501 \mathrm{MHz}$, DMSO- $\left.d_{6}\right) \delta \mathrm{ppm} 7.34(\mathrm{dd}, J=7.6,1.5 \mathrm{~Hz}, 1 \mathrm{H}), 7.15(\mathrm{dtd}, J=25.3,7.5,1.6 \mathrm{~Hz}, 2 \mathrm{H}), 7.07-7.01(\mathrm{~m}$, $1 \mathrm{H}), 5.31(\mathrm{dd}, J=6.7,3.1 \mathrm{~Hz}, 1 \mathrm{H}), 4.92(\mathrm{dd}, J=8.4,6.6 \mathrm{~Hz}, 1 \mathrm{H}), 4.27-4.12(\mathrm{~m}, 2 \mathrm{H}), 3.75(\mathrm{t}, J=7.7$ $\mathrm{Hz}, 1 \mathrm{H}), 3.51(\mathrm{t}, J=8.1 \mathrm{~Hz}, 1 \mathrm{H}), 3.04(\mathrm{dd}, J=7.6,3.1 \mathrm{~Hz}, 1 \mathrm{H}), 2.06(\mathrm{tt}, J=8.5,5.4 \mathrm{~Hz}, 1 \mathrm{H}), 1.24(\mathrm{t}, J=$ $7.1 \mathrm{~Hz}, 3 \mathrm{H}), 0.95(\mathrm{~s}, 9 \mathrm{H}), 1.00-0.78(\mathrm{~m}, 2 \mathrm{H}), 0.81-0.68(\mathrm{~m}, 1 \mathrm{H}), 0.64-0.55(\mathrm{~m}, 1 \mathrm{H}) . \mathrm{MS}\left(\mathrm{ESI}^{+}\right) \mathrm{m} / z$ $361.2(\mathrm{M}+\mathrm{H})^{+}$.

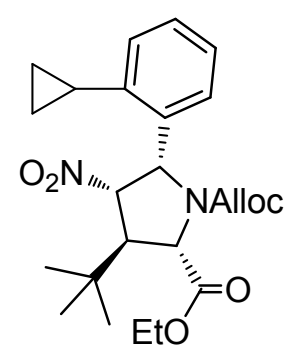

\section{1-allyl 2-ethyl (2S,3R,4S,5S)-3-(tert-butyl)-5-(2-cyclopropylphenyl)-4-nitropyrrolidine-1,2-}

\section{dicarboxylate (60)}

Compound 59 (2.00 g, $5.55 \mathrm{mmol})$ was dissolved in toluene $(10 \mathrm{~mL})$ and saturated aqueous $\mathrm{NaHCO}_{3}(10$ $\mathrm{mL}$ ) was added followed by allyl chloroformate $(0.708 \mathrm{~mL}, 6.66 \mathrm{mmol})$. The reaction was stirred at ambient temperature for 17 hours, at which point it was complete. The reaction was diluted with dichloromethane $(50 \mathrm{~mL})$ and washed with $1 \mathrm{M}$ aqueous $\mathrm{HCl}(2 \times 50 \mathrm{~mL})$ and brine. The organic layer was dried over sodium sulfate, filtered, and concentrated to provide the crude product. The residue was purified by silica gel chromatography ( $5 \%$ ethyl acetate in dichloromethane) to provide the title compound (1.76 g,71\%). ${ }^{1} \mathrm{H}$ NMR $\left(400 \mathrm{MHz}, \mathrm{DMSO}-d_{6}, 120^{\circ} \mathrm{C}\right) \delta \mathrm{ppm} 7.78(\mathrm{dd}, \mathrm{J}=7.6,1.7 \mathrm{~Hz}, 1 \mathrm{H})$, 7.17 - $7.04(\mathrm{~m}, 2 \mathrm{H}), 7.01-6.95(\mathrm{~m}, 1 \mathrm{H}), 6.00(\mathrm{~d}, \mathrm{~J}=8.6 \mathrm{~Hz}, 1 \mathrm{H}), 5.67$ (ddt, J = 17.2, 10.4, 5.2 Hz, 1H), $5.56(\mathrm{dd}, \mathrm{J}=8.6,2.5 \mathrm{~Hz}, 1 \mathrm{H}), 5.05-4.89(\mathrm{~m}, 2 \mathrm{H}), 4.60(\mathrm{~d}, \mathrm{~J}=3.4 \mathrm{~Hz}, 1 \mathrm{H}), 4.41(\mathrm{dq}, \mathrm{J}=5.2,1.5 \mathrm{~Hz}, 2 \mathrm{H})$, $4.27(\mathrm{qd}, \mathrm{J}=7.1,1.0 \mathrm{~Hz}, 2 \mathrm{H}), 3.05(\mathrm{ddd}, \mathrm{J}=3.3,2.5,0.7 \mathrm{~Hz}, 1 \mathrm{H}), 2.06(\mathrm{tt}, \mathrm{J}=8.5,5.4 \mathrm{~Hz}, 1 \mathrm{H}), 1.30$ (t, J $=7.1 \mathrm{~Hz}, 3 \mathrm{H}), 1.04(\mathrm{~s}, 9 \mathrm{H}), 1.02-0.88(\mathrm{~m}, 2 \mathrm{H}), 0.85-0.75(\mathrm{~m}, 1 \mathrm{H}), 0.54(\mathrm{dtd}, \mathrm{J}=7.9,5.1,2.9 \mathrm{~Hz}, 1 \mathrm{H})$; MS (ESI+) $m / z 445(\mathrm{M}+\mathrm{H})^{+}$. 


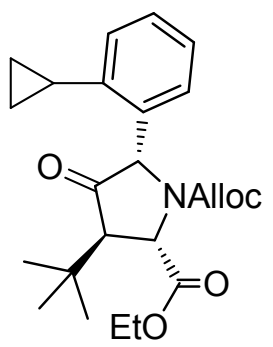

1-allyl 2-ethyl (2S,3R,5S)-3-(tert-butyl)-5-(2-cyclopropylphenyl)-4-oxopyrrolidine-1,2-dicarboxylate (61)

Compound 60 (1.79 g, $3.93 \mathrm{mmol}$ ) was dissolved in $44 \mathrm{~mL}$ of ethanol and the solution was heated to 75 ${ }^{\circ} \mathrm{C}$. A separate solution of $\mathrm{CrCl}_{2}$ was prepared by dissolving pyridinium dichromate $(4.066 \mathrm{~g}, 10.81$ $\mathrm{mmol})$ in $88 \mathrm{~mL}$ of $6 \mathrm{M}$ aqueous $\mathrm{HCl}$ and adding $\mathrm{Zn}(7.904 \mathrm{~g}, 119 \mathrm{mmol})$ in portions while cooling in an ice bath. The suspension was stirred until all $\mathrm{Zn}$ dissolved, leaving a brilliant blue solution. $\mathrm{The}^{\mathrm{CrCl}_{2}}$ solution was transferred via cannula over 15 minutes to the solution of starting material and heating was continued for 16 hours. The temperature was maintained between $70{ }^{\circ} \mathrm{C}$ and $75{ }^{\circ} \mathrm{C}$ during the addition, and was maintained between $75^{\circ} \mathrm{C}$ and $80{ }^{\circ} \mathrm{C}$ overnight (total 16 hours). The reaction mixture was cooled to room temperature, diluted with water and extracted with dichloromethane $(3 \mathrm{x} 200 \mathrm{~mL})$. The combined extracts were washed with brine, and dried over sodium sulfate, filtered, and concentrated. The crude residue was dissolved ethanol $(6 \mathrm{~mL})$. A separate solution of $\mathrm{HCl} /$ ethanol was prepared by addition of $1 \mathrm{~mL}$ of acetyl chloride to $4 \mathrm{~mL}$ of ethanol at $0{ }^{\circ} \mathrm{C}$, and poured into the reaction flask and the mixture was heated to $45^{\circ} \mathrm{C}$ for 1 hour. The reaction mixture was concentrated in vacuo and loaded onto a $40 \mathrm{~g}$ silica gel column and was eluted with 5\% ethyl acetate in dichloromethane over to provide the title compound (518.9 mg, 32\%). ${ }^{1} \mathrm{H}$ NMR $\left(400 \mathrm{MHz}\right.$, DMSO- $\left.d_{6}, 120{ }^{\circ} \mathrm{C}\right) \delta \mathrm{ppm} 7.69(\mathrm{dd}, \mathrm{J}=7.1,2.0 \mathrm{~Hz}$, 1H), 7.19 - $7.05(\mathrm{~m}, 3 \mathrm{H}), 5.74$ - $5.63(\mathrm{~m}, 2 \mathrm{H}), 5.07$ - $4.94(\mathrm{~m}, 2 \mathrm{H}), 4.65(\mathrm{~d}, \mathrm{~J}=5.0 \mathrm{~Hz}, 1 \mathrm{H}), 4.45(\mathrm{dd}, \mathrm{J}=$ 5.3, $1.8 \mathrm{~Hz}, 2 \mathrm{H}), 4.28$ - $4.18(\mathrm{~m}, 2 \mathrm{H}), 2.63(\mathrm{dd}, \mathrm{J}=5.0,0.9 \mathrm{~Hz}, 1 \mathrm{H}), 2.13(\mathrm{tt}, \mathrm{J}=8.5,5.4 \mathrm{~Hz}, 1 \mathrm{H}), 1.26$ $(\mathrm{td}, \mathrm{J}=7.1,0.8 \mathrm{~Hz}, 3 \mathrm{H}), 1.07(\mathrm{~d}, \mathrm{~J}=0.8 \mathrm{~Hz}, 9 \mathrm{H}), 0.95-0.83(\mathrm{~m}, 2 \mathrm{H}), 0.83-0.72(\mathrm{~m}, 1 \mathrm{H}), 0.52$ (dddd, J $=7.8,6.6,3.9,1.5 \mathrm{~Hz}, 1 \mathrm{H})$; $\mathrm{MS}(\mathrm{ESI}+) \mathrm{m} / \mathrm{z} 414(\mathrm{M}+\mathrm{H})^{+}$.

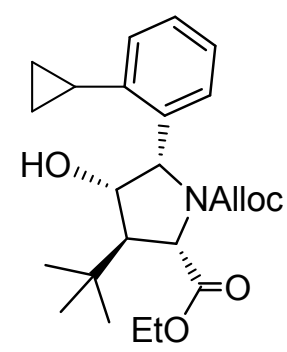

1-allyl 2-ethyl (2S,3R,4S,5S)-3-(tert-butyl)-5-(2-cyclopropylphenyl)-4-hydroxypyrrolidine-1,2dicarboxylate (62) 
Compound $61(504.6 \mathrm{mg}, 1.220 \mathrm{mmol})$ was dissolved in ethanol $(5 \mathrm{~mL})$, and the reaction was cooled to 0 ${ }^{\circ} \mathrm{C}$. Sodium borohydride $(75.0 \mathrm{mg}, 1.982 \mathrm{mmol})$ was added and the reaction was stirred at $0{ }^{\circ} \mathrm{C}$ for 1 hour, and warmed to ambient temperature for another 1 hour. The solvent was removed in vacuo and the residue was extracted with ethyl acetate $(50 \mathrm{~mL})$, washed with saturated aqueous $\mathrm{NaHCO}_{3}(50 \mathrm{~mL})$ and brine $(50 \mathrm{~mL})$, dried over $\mathrm{Na}_{2} \mathrm{SO}_{4}$, filtered, and concentrated to provide crude product, which was purified by silica gel chromatography ( $5 \%$ ethyl acetate in dichloromethane) to provide the title compound ( 280 mg, 55\%). ${ }^{1} \mathrm{H}$ NMR (400 MHz, DMSO- $\left.d_{6}, 120^{\circ} \mathrm{C}\right) \delta \mathrm{ppm} 7.89-7.83(\mathrm{~m}, 1 \mathrm{H}), 7.10-7.04(\mathrm{~m}, 2 \mathrm{H}), 7.01$ - $6.95(\mathrm{~m}, 1 \mathrm{H}), 5.72$ - $5.60(\mathrm{~m}, 1 \mathrm{H}), 5.51(\mathrm{~d}, \mathrm{~J}=6.2 \mathrm{~Hz}, 1 \mathrm{H}), 5.03-4.91(\mathrm{~m}, 2 \mathrm{H}), 4.45$ (td, J = 5.6, $2.9 \mathrm{~Hz}$, $1 \mathrm{H}), 4.36(\mathrm{td}, \mathrm{J}=3.5,2.1 \mathrm{~Hz}, 3 \mathrm{H}), 4.18(\mathrm{q}, \mathrm{J}=7.1 \mathrm{~Hz}, 2 \mathrm{H}), 3.71(\mathrm{~s}, 1 \mathrm{H}), 2.31(\mathrm{t}, \mathrm{J}=3.4 \mathrm{~Hz}, 1 \mathrm{H}), 1.97$ (tt, $\mathrm{J}=7.9,5.4 \mathrm{~Hz}, 1 \mathrm{H}), 1.25(\mathrm{t}, \mathrm{J}=7.1 \mathrm{~Hz}, 3 \mathrm{H}), 1.01(\mathrm{~s}, 9 \mathrm{H}), 0.88(\mathrm{ttd}, \mathrm{J}=12.7,8.9,5.6 \mathrm{~Hz}, 2 \mathrm{H}), 0.73(\mathrm{dtd}$, $\mathrm{J}=9.4,5.4,3.6 \mathrm{~Hz}, 1 \mathrm{H}), 0.61-0.49(\mathrm{~m}, 1 \mathrm{H}) ; \mathrm{MS}(\mathrm{ESI}+) \mathrm{m} / \mathrm{z} 416(\mathrm{M}+\mathrm{H})^{+}$.

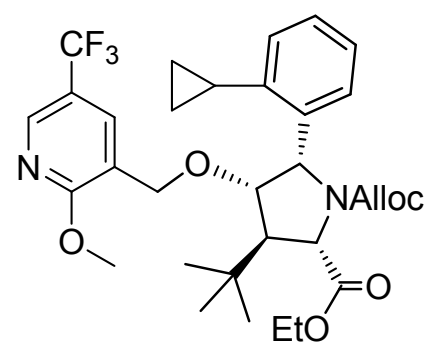

1-allyl 2-ethyl (2S,3R,4S,5S)-3-(tert-butyl)-5-(2-cyclopropylphenyl)-4-((2-methoxy-5(trifluoromethyl)pyridin-3-yl)methoxy)pyrrolidine-1,2-dicarboxylate (63)

Compound $62(275.0 \mathrm{mg}, 0.662 \mathrm{mmol}$ ) and 3-(bromomethyl)-2-methoxy-5-(trifluoromethyl)pyridine (312.1 mg, $1.156 \mathrm{mmol}$ ) were dissolved in dimethylformamide $(2.6 \mathrm{~mL})$. The reaction was cooled to 0 ${ }^{\circ} \mathrm{C}$, and potassium tert-butoxide ( $1 \mathrm{M}$ in tetrahydrofuran, $\left.0.80 \mathrm{~mL}, 0.80 \mathrm{mmol}\right)$ was added dropwise. The reaction mixture was stirred at ambient temperature for 1 hour. The reaction mixture was poured into saturated aqueous $\mathrm{NH}_{4} \mathrm{Cl}(35 \mathrm{~mL})$, extracted with methyl tert-butyl ether $(3 \times 35 \mathrm{~mL})$, and purified by silica gel chromatography ( $5 \%$ ethyl acetate in dichloromethane) to provide the title compound (350.8 mg, 88\%). ${ }^{1} \mathrm{H}$ NMR (400 MHz, DMSO- $\left.d_{6}, 120{ }^{\circ} \mathrm{C}\right) \delta \mathrm{ppm} 8.33-8.25(\mathrm{~m}, 1 \mathrm{H}), 7.88(\mathrm{dd}, \mathrm{J}=7.3,2.1 \mathrm{~Hz}$, 1H), 7.20 - $7.15(\mathrm{~m}, 1 \mathrm{H}), 7.02(\mathrm{ddd}, \mathrm{J}=7.0,4.5,1.9 \mathrm{~Hz}, 2 \mathrm{H}), 6.94(\mathrm{dd}, \mathrm{J}=6.9,2.1 \mathrm{~Hz}, 1 \mathrm{H}), 5.73$ - 5.58 $(\mathrm{m}, 2 \mathrm{H}), 5.05-4.90(\mathrm{~m}, 2 \mathrm{H}), 4.52(\mathrm{~d}, \mathrm{~J}=2.0 \mathrm{~Hz}, 1 \mathrm{H}), 4.41-4.37(\mathrm{~m}, 2 \mathrm{H}), 4.37$ - $4.33(\mathrm{~m}, 1 \mathrm{H}), 4.27(\mathrm{~d}, \mathrm{~J}$ $=13.8 \mathrm{~Hz}, 1 \mathrm{H}), 4.11(\mathrm{dtd}, \mathrm{J}=8.0,6.9,1.2 \mathrm{~Hz}, 2 \mathrm{H}), 3.87-3.79(\mathrm{~m}, 4 \mathrm{H}), 2.52(\mathrm{t}, \mathrm{J}=1.3 \mathrm{~Hz}, 1 \mathrm{H}), 1.99$ (td, $\mathrm{J}=8.4,4.2 \mathrm{~Hz}, 1 \mathrm{H}), 1.15(\mathrm{td}, \mathrm{J}=7.1,0.7 \mathrm{~Hz}, 3 \mathrm{H}), 1.05(\mathrm{~s}, 9 \mathrm{H}), 0.96-0.83(\mathrm{~m}, 2 \mathrm{H}), 0.59(\mathrm{dd}, \mathrm{J}=5.2,2.6$ $\mathrm{Hz}, 2 \mathrm{H}) ; \mathrm{MS}(\mathrm{ESI}+) \mathrm{m} / z 605(\mathrm{M}+\mathrm{H})^{+}$. 


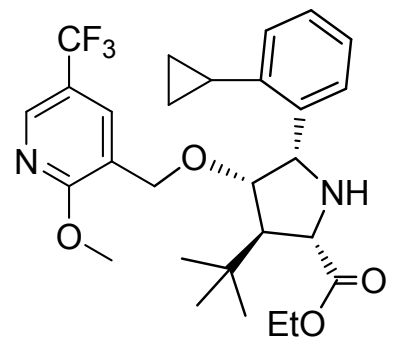

ethyl (2S,3R,4S,5S)-3-(tert-butyl)-5-(2-cyclopropylphenyl)-4-((2-methoxy-5(trifluoromethyl)pyridin-3-yl)methoxy)pyrrolidine-2-carboxylate (64)

Compound 63 (350 mg, $0.579 \mathrm{mmol})$ was dissolved in ethyl acetate $(2.5 \mathrm{~mL})$ and dichloromethane $(2.5$ $\mathrm{mL}$ ) and treated with 1,3-dimethylpyrimidine-2,4,6(1H,3H,5H)-trione (183.2 $\mathrm{mg}, 1.173 \mathrm{mmol})$ and tetrakis(triphenylphosphine)palladium $(7.2 \mathrm{mg}, 6.23 \mu \mathrm{mol})$. The reaction mixture was stirred at ambient temperature. After 1 hour, the reaction mixture was diluted with methyl tert-butyl ether $(50 \mathrm{~mL})$ and stirred with $15 \mathrm{~mL}$ of $10 \%$ aqueous $\mathrm{Na}_{2} \mathrm{CO}_{3}(50 \mathrm{~mL})$ solution for 30 minutes. The phases were separated, and the organic layer was washed with brine, dried over $\mathrm{Na}_{2} \mathrm{SO}_{4}$, filtered, and concentrated. The residue was purified by silica gel chromatography, eluting with $5 \%$ to $10 \%$ ethyl acetate in dichloromethane to provide the title compound (220.4 mg, 73\%). ${ }^{1} \mathrm{H}$ NMR $\left(400 \mathrm{MHz}, \mathrm{DMSO}-d_{6}, 120{ }^{\circ} \mathrm{C}\right) \delta \mathrm{ppm} 8.29(\mathrm{~s}$, 1H), 7.59 (dd, J = 7.5, $1.7 \mathrm{~Hz}, 1 \mathrm{H}), 7.36(\mathrm{~d}, \mathrm{~J}=2.4 \mathrm{~Hz}, 1 \mathrm{H}), 7.12-7.00(\mathrm{~m}, 2 \mathrm{H}), 6.95$ (dd, J = 7.2, 1.7 Hz, 1H), $4.65(\mathrm{~d}, \mathrm{~J}=4.4 \mathrm{~Hz}, 1 \mathrm{H}), 4.23(\mathrm{~d}, \mathrm{~J}=13.8 \mathrm{~Hz}, 1 \mathrm{H}), 4.19-4.08(\mathrm{~m}, 3 \mathrm{H}), 3.85(\mathrm{~s}, 3 \mathrm{H}), 3.80(\mathrm{~d}, \mathrm{~J}=$ $13.8 \mathrm{~Hz}, 1 \mathrm{H}), 3.66(\mathrm{~d}, \mathrm{~J}=6.4 \mathrm{~Hz}, 1 \mathrm{H}), 2.40(\mathrm{dd}, \mathrm{J}=6.3,1.6 \mathrm{~Hz}, 1 \mathrm{H}), 1.99(\mathrm{tt}, \mathrm{J}=8.5,5.5 \mathrm{~Hz}, 1 \mathrm{H}), 1.21$ $(\mathrm{t}, \mathrm{J}=7.1 \mathrm{~Hz}, 3 \mathrm{H}), 1.01(\mathrm{~s}, 10 \mathrm{H}), 0.90(\mathrm{dq}, \mathrm{J}=8.3,2.1 \mathrm{~Hz}, 2 \mathrm{H}), 0.68$ - 0.55 (m, 2H); MS (ESI+) $m / z 521$ $(\mathrm{M}+\mathrm{H})^{+}$.

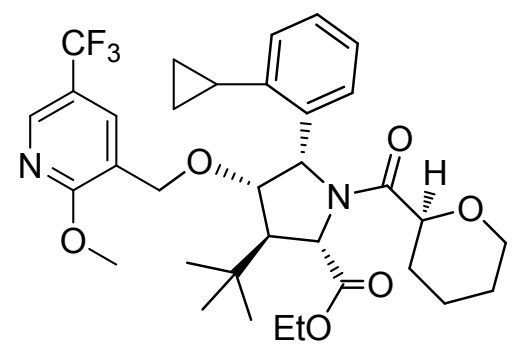

ethyl (2S,3R,4S,5S)-3-(tert-butyl)-5-(2-cyclopropylphenyl)-4-((2-methoxy-5(trifluoromethyl)pyridin-3-yl)methoxy)-1-((S)-tetrahydro-2H-pyran-2-carbonyl)pyrrolidine-2carboxylate (65)

Tetrahydropyran-2-carboxylic acid $(53.7 \mathrm{mg}, 0.413 \mathrm{mmol})$ was dissolved in dichloromethane $(2 \mathrm{~mL})$. Oxalyl chloride ( $2 \mathrm{M}$ in dichloromethane, $250 \mu \mathrm{L}, 0.50 \mathrm{mmol}$ ) was added followed by dimethylformamide $(25 \mu \mathrm{L})$. The reaction was stirred at room temperature for 3 hours, at which point it was concentrated, redissolved in dichloromethane $(1 \mathrm{~mL})$, and concentrated again. The residue was taken up in 
dichloromethane $(3 \times 1 \mathrm{~mL})$ and was added to a solution of Compound $64(90.4 \mathrm{mg}, 0.174 \mathrm{mmol})$ and triethylamine $(100 \mu \mathrm{L}, 0.717 \mathrm{mmol})$ in dichloromethane $(3 \mathrm{~mL})$. The reaction was stirred at ambient temperature for 17 hours. After this time, the mixture was diluted with dichloromethane $(100 \mathrm{~mL})$ and washed twice with saturated aqueous $\mathrm{NaHCO}_{3}$ and once with brine. The organic layer was dried over $\mathrm{Na}_{2} \mathrm{SO}_{4}$, filtered, and concentrated. The residue was purified by reverse-phase preparative HPLC on a Phenomenex ${ }^{\circledR}$ Luna ${ }^{\circledR}$ C 8(2) $5 \mu \mathrm{m} 100 \AA$ AXIA $^{\mathrm{TM}}$ column $(30 \mathrm{~mm} \times 75 \mathrm{~mm})$. A gradient of acetonitrile (A) and $0.1 \%$ trifluoroacetic acid in water (B) was used, at a flow rate of $50 \mathrm{~mL} /$ minute $(0-0.5$ minutes $10 \%$ A, 0.5-7.0 minutes linear gradient 10-95\% A, 7.0-10.0 minutes 95\% A, 10.0-12.0 minutes linear gradient $95-10 \% \mathrm{~A})$. The title compound was the second eluting diastereomer and was isolated as the TFA salt (43.7 mg, 34\%). ${ }^{1} \mathrm{H}$ NMR (400 MHz, DMSO- $\left.d_{6}, 120^{\circ} \mathrm{C}\right) \delta \mathrm{ppm} 8.27(\mathrm{~s}, 1 \mathrm{H}), 7.96(\mathrm{dd}, \mathrm{J}=7.6$, $1.7 \mathrm{~Hz}, 1 \mathrm{H}), 7.16(\mathrm{~d}, \mathrm{~J}=2.5 \mathrm{~Hz}, 1 \mathrm{H}), 7.06(\mathrm{t}, \mathrm{J}=9.6 \mathrm{~Hz}, 2 \mathrm{H}), 6.95(\mathrm{~d}, \mathrm{~J}=7.1 \mathrm{~Hz}, 1 \mathrm{H}), 5.90(\mathrm{~s}, 1 \mathrm{H}), 4.72$ $(\mathrm{s}, 1 \mathrm{H}), 4.36(\mathrm{~d}, \mathrm{~J}=5.6 \mathrm{~Hz}, 1 \mathrm{H}), 4.28(\mathrm{~d}, \mathrm{~J}=13.9 \mathrm{~Hz}, 1 \mathrm{H}), 4.08(\mathrm{qd}, \mathrm{J}=7.1,3.0 \mathrm{~Hz}, 2 \mathrm{H}), 3.89$ - $3.80(\mathrm{~m}$, 5H), $3.75(\mathrm{~d}, \mathrm{~J}=11.4 \mathrm{~Hz}, 1 \mathrm{H}), 3.45(\mathrm{~s}, 1 \mathrm{H}), 2.44(\mathrm{~d}, \mathrm{~J}=1.8 \mathrm{~Hz}, 1 \mathrm{H}), 2.06$ - $1.94(\mathrm{~m}, 1 \mathrm{H}), 1.72$ - $1.26(\mathrm{~m}$, $6 \mathrm{H}), 1.13(\mathrm{t}, \mathrm{J}=7.1 \mathrm{~Hz}, 3 \mathrm{H}), 1.04$ (s, 9H), 0.99 - $0.84(\mathrm{~m}, 2 \mathrm{H}), 0.80$ - 0.67 (m, 1H), 0.57 - 0.46 (m, 1H); MS (ESI+) $m / z 633(\mathrm{M}+\mathrm{H})^{+}$.

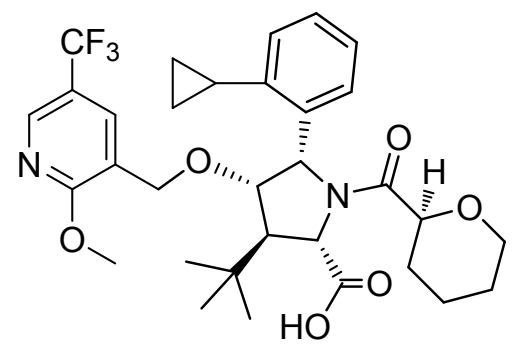

(2S,3R,4S,5S)-3-tert-butyl-5-(2-cyclopropylphenyl)-4-\{[2-methoxy-5-(trifluoromethyl)pyridin-3yl]methoxy\}-1-[(2S)-oxane-2-carbonyl]pyrrolidine-2-carboxylic acid (15)

Compound 65 (40.7 mg, $0.055 \mathrm{mmol})$ was dissolved in methanol $(1 \mathrm{~mL})$, and tetrahydrofuran $(1 \mathrm{~mL})$. Aqueous $\mathrm{LiOH}(1 \mathrm{M}, 0.5 \mathrm{~mL}, 0.5 \mathrm{mmol})$ was added and the reaction was heated to $50{ }^{\circ} \mathrm{C}$ for 16 hours. The reaction mixture was quenched by the addition of $1 \mathrm{M}$ aqueous $\mathrm{HCl}(0.5 \mathrm{~mL})$, concentrated and purified by reverse-phase preparative HPLC on a Phenomenex ${ }^{\circledR}$ Luna ${ }^{\circledR}$ C8(2) $5 \mu$ m $100 \AA$ AXIA ${ }^{\mathrm{TM}}$ column $(30 \mathrm{~mm} \times 75 \mathrm{~mm})$. A gradient of acetonitrile (A) and $0.1 \%$ trifluoroacetic acid in water (B) was used, at a flow rate of $50 \mathrm{~mL} /$ minute $(0-0.5$ minutes $10 \% \mathrm{~A}, 0.5-7.0$ minutes linear gradient $10-95 \% \mathrm{~A}$, 7.0-10.0 minutes $95 \%$ A, 10.0-12.0 minutes linear gradient $95-10 \%$ A) to provide the title compound (27.3 mg, 70\%) as the TFA salt. ${ }^{1} \mathrm{H}$ NMR $\left(400 \mathrm{MHz}, \mathrm{DMSO}-d_{6}, 120^{\circ} \mathrm{C}\right) \delta \mathrm{ppm} 8.27(\mathrm{~d}, \mathrm{~J}=2.4 \mathrm{~Hz}, 1 \mathrm{H})$, $8.02(\mathrm{dd}, \mathrm{J}=7.5,1.8 \mathrm{~Hz}, 1 \mathrm{H}), 7.13(\mathrm{~s}, 1 \mathrm{H}), 7.11-6.99(\mathrm{~m}, 2 \mathrm{H}), 6.94(\mathrm{dd}, \mathrm{J}=7.3,1.7 \mathrm{~Hz}, 1 \mathrm{H}), 5.90$ (s, 1H), $4.69(\mathrm{~s}, 1 \mathrm{H}), 4.35(\mathrm{~d}, \mathrm{~J}=5.8 \mathrm{~Hz}, 1 \mathrm{H}), 4.31(\mathrm{~d}, \mathrm{~J}=14.0 \mathrm{~Hz}, 1 \mathrm{H}), 3.95$ - $3.84(\mathrm{~m}, 5 \mathrm{H}), 3.76(\mathrm{~d}, \mathrm{~J}=$ 
$11.5 \mathrm{~Hz}, 1 \mathrm{H}), 3.47(\mathrm{~s}, 1 \mathrm{H}), 2.50(\mathrm{~d}, \mathrm{~J}=1.8 \mathrm{~Hz}, 1 \mathrm{H}), 2.07-1.94(\mathrm{~m}, 1 \mathrm{H}), 1.71-1.25(\mathrm{~m}, 6 \mathrm{H}), 1.04(\mathrm{~s}, 9 \mathrm{H})$, $0.98-0.84(\mathrm{~m}, 2 \mathrm{H}), 0.78-0.65(\mathrm{~m}, 1 \mathrm{H}), 0.49(\mathrm{dtd}, \mathrm{J}=9.1,5.3,3.0 \mathrm{~Hz}, 1 \mathrm{H}) ;$ MS (ESI+) $\mathrm{m} / \mathrm{z} 605.1$ $(\mathrm{M}+\mathrm{H})^{+}$.

Scheme S9. Discovery Synthesis of Compound 16

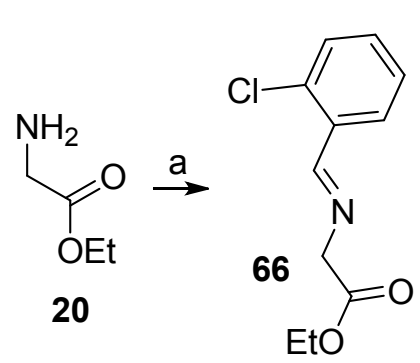<smiles>CCOC(=O)[C@H]1N[C@@H](c2ccccc2Cl)[C@H]([N+](=O)[O-])[C@@H]1C(C)(C)C</smiles><smiles>COc1ncc(C(F)(F)F)cc1CO[C@@H]1[C@H](c2ccccc2Cl)N(C(=O)[C@@H]2CCCCO2)[C@H](C(=O)O)[C@H]1C(C)(C)C</smiles>

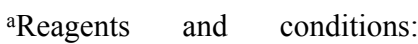

(a) $\mathrm{MgSO}_{4}, \quad \mathrm{TEA}, \quad$ 2-methylbenzaldehyde, DCM;

(b) $\quad(2-(\operatorname{Bis}(3,5-$ bis(trifluoromethyl)phenyl)phosphino)-3-((S)-4-isopropyl-4,5-dihydrooxazol-2-yl)cyclopenta-2,4-dien-1-yl)(cyclopenta-2,4-dien1-yl)iron, $\mathrm{Cu}(\mathrm{OTf})_{2}$, KOtBu, (E)-3,3-dimethyl-1-nitrobut-1-ene, THF; (c) allyl chloroformate, toluene; (d) PDC, 6N HCl, Zn dust, (e) $\mathrm{NaBH}_{4}, \mathrm{EtOH}$; (f) $\mathrm{KOtBu}, 3$-(bromomethyl)-2-methoxy-5-(trifluoromethyl)pyridine, DMF; (g) 1,3-Dimethlbarbituric acid, $\mathrm{Pd}\left(\mathrm{PPh}_{3}\right)_{4}$, EtOAc/DCM; (h) Tetrahydropyran-2-carboxylic acid, oxalyl chloride, DMF, DCM; (i) LiOH, MeOH/THF.

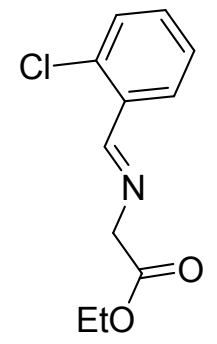

\section{(E)-ethyl 2-((2-chlorobenzylidene)amino)acetate (66)}

A mixture of ethyl 2-aminoacetate hydrochloride $(1.85 \mathrm{~g}, 13.25 \mathrm{mmol})$ and magnesium sulfate (3.19 g, $26.5 \mathrm{mmol})$ in dichloromethane $(22.09 \mathrm{~mL})$ (anhydrous) was treated with triethylamine (1.847 mL, $13.25 \mathrm{mmol})$, stirred for 30 minutes, and treated with the 2-chlorobenzaldehyde (1.86 $\mathrm{g}, 13.25 \mathrm{mmol}$ ) as a solution in $3 \mathrm{~mL}$ of dichloromethane. The reaction was stirred at ambient temperature overnight. The solid material was filtered and the filtrate was concentrated.

Toluene $(5 \mathrm{~mL})$ was added, the mixture was filtered again and concentrated, giving $(E)$-ethyl 2((2-chlorobenzylidene)amino)acetate (2.76 g, $12.23 \mathrm{mmol}, 92 \%$ yield) which was used directly in the next step. ${ }^{1} \mathrm{H}$ NMR $\left(400 \mathrm{MHz}, \mathrm{CDCl}_{3}\right) \delta \mathrm{ppm} 8.77(\mathrm{~d}, \mathrm{~J}=1.5 \mathrm{~Hz}, 1 \mathrm{H}), 8.19-8.04(\mathrm{~m}$, 
1H), 7.45 - $7.39(\mathrm{~m}, 2 \mathrm{H}), 7.34$ (ddd, J = 8.3, 6.0, $2.6 \mathrm{~Hz}, 1 \mathrm{H}), 4.48(\mathrm{~d}, \mathrm{~J}=1.5 \mathrm{~Hz}, 2 \mathrm{H}), 4.28$ (q, J $=7.2 \mathrm{~Hz}, 2 \mathrm{H}), 1.34(\mathrm{t}, \mathrm{J}=7.1 \mathrm{~Hz}, 3 \mathrm{H})$.

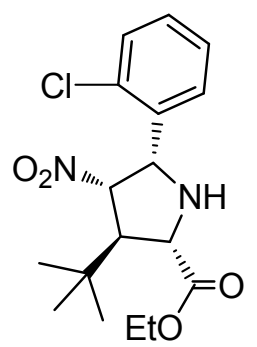

(2S,3R,4S,5S)-ethyl 3-(tert-butyl)-5-(2-chlorophenyl)-4-nitropyrrolidine-2-carboxylate (67)

(2-(Bis(3,5-bis(trifluoromethyl)phenyl)phosphino)-3-((S)-4-isopropyl-4,5-dihydrooxazol-2-yl)cyclopenta2,4-dien-1-yl)(cyclopenta-2,4-dien-1-yl)iron (0.175 g, $0.232 \mathrm{mmol})$ and copper (I) triflate dimer, benzene complex (0.047 g, $0.093 \mathrm{mmol})$ were dissolved in tetrahydrofuran $(19.36 \mathrm{~mL} \mathrm{~mL})$ that had been sparged with an $\mathrm{N}_{2}$ stream for 1 hour. The resulting mixture was stirred for 1 hour at ambient temperature (continue nitrogen sparge), and (E)-ethyl 2-((2-chlorobenzylidene)amino)acetate (66, $2.75 \mathrm{~g}, 12.19 \mathrm{mmol})$ was added as a solution in $2 \mathrm{~mL}$ of tetrahydrofuran and the resulting solution was cooled to $<5{ }^{\circ} \mathrm{C}$ in an ice-water bath. Potassium 2-methylpropan-2-olate in tetrahydrofuran $(0.209 \mathrm{~mL}, 0.209 \mathrm{mmol})$ was added dropwise, followed by addition of neat $(E)$-3,3-dimethyl-1-nitrobut-1-ene (1.5 g, $11.61 \mathrm{mmol})$ over 20 minutes, maintaining a temperature less than $7^{\circ} \mathrm{C}$. The reaction mixture was stirred for 1 hour at $0{ }^{\circ} \mathrm{C}$ and quenched with $60 \mathrm{~mL}$ of saturated aqueous ammonium chloride and $100 \mathrm{~mL}$ of ethyl acetate and warmed to ambient temperature. The organic layer was separated and washed with saturated aqueous ammonium chloride $(2 \times 50 \mathrm{~mL})$ and brine and filtered through a pad of silica gel. The organic layer was concentrated. Heptane $(70 \mathrm{~mL})$ was added, and the resulting precipitate was filtered. The filtrate was purified by chromatography using a $40 \mathrm{~g}$ silica gel cartridge eluting with a gradient of $0-60 \%$ heptanes/ethyl acetate over a period of 20 minutes to provide additional product ( $2 S, 3 R, 4 S, 5 S)$-ethyl 3(tert-butyl)-5-(2-chlorophenyl)-4-nitropyrrolidine-2-carboxylate (2.85 g, $8.03 \mathrm{mmol}, 69.2 \%$ yield). ${ }^{1} \mathrm{H}$ NMR (400 MHz, DMSO- $\left.d_{6}\right) \delta$ ppm 7.56 - $7.48(\mathrm{~m}, 1 \mathrm{H}), 7.45-7.38(\mathrm{~m}, 1 \mathrm{H}), 7.35$ - $7.24(\mathrm{~m}, 2 \mathrm{H}), 5.25$ $(\mathrm{dd}, \mathrm{J}=6.7,3.0 \mathrm{~Hz}, 1 \mathrm{H}), 4.71(\mathrm{t}, \mathrm{J}=7.0 \mathrm{~Hz}, 1 \mathrm{H}), 4.15-4.24(\mathrm{~m}, 2 \mathrm{H}), 3.78(\mathrm{t}, \mathrm{J}=7.3 \mathrm{~Hz}, 1 \mathrm{H}), 3.68(\mathrm{t}, \mathrm{J}=$ $7.3 \mathrm{~Hz}, 1 \mathrm{H}), 3.07$ (dd, J = 7.4, 3.0 Hz, 1H), 1.24 (t, J = 7.1 Hz, 3H), 0.94 (s, 9H); MS (APCI+) m/z 355 $(\mathrm{M}+\mathrm{H})^{+}$. 
<smiles>COc1ncc(C(F)(F)F)cc1CO[C@@H]1[C@H](c2ccccc2Cl)N(C(=O)[C@H]2CCCCO2)[C@H](C(=O)O)[C@@H]1C(C)(C)C</smiles>

(2S,3R,4S,5S)-3-tert-butyl-5-(2-chlorophenyl)-4-\{[2-methoxy-5-(trifluoromethyl)pyridin-3yl]methoxy\}-1-[(2S)-oxane-2-carbonyl]pyrrolidine-2-carboxylic acid (16)

The title compound was prepared using the procedures described for compound 15 substituting compound 67 for compound 59. ${ }^{1} \mathrm{H}$ NMR (400 MHz, DMSO- $\left.d_{6}\right) \delta \mathrm{ppm} 8.31(\mathrm{~s}, 1 \mathrm{H}), 8.28(\mathrm{~s}, 1 \mathrm{H}), 7.27(\mathrm{~s}, 1 \mathrm{H})$, $7.17(\mathrm{~s}, 2 \mathrm{H}), 7.09$ (d, J = 2.2 Hz, 1H), $5.80(\mathrm{~s}, 1 \mathrm{H}), 4.63$ - $4.54(\mathrm{~m}, 1 \mathrm{H}), 4.37$ (d, J = 14.0 Hz, 1H), $4.32(\mathrm{~d}$, $\mathrm{J}=6.3 \mathrm{~Hz}, 1 \mathrm{H}), 4.00(\mathrm{~d}, \mathrm{~J}=14.0 \mathrm{~Hz}, 1 \mathrm{H}), 3.88(\mathrm{~s}, 3 \mathrm{H}), 3.80(\mathrm{~d}, \mathrm{~J}=11.2 \mathrm{~Hz}, 1 \mathrm{H}), 3.25(\mathrm{bs}, 2 \mathrm{H}), 2.58$ (s, 1H), $1.70(\mathrm{~s}, 1 \mathrm{H}), 1.54(\mathrm{~s}, 2 \mathrm{H}), 1.37(\mathrm{bs}, 3 \mathrm{H}), 1.01(\mathrm{~s}, 9 \mathrm{H})$; MS (ESI+) $m / z 599(\mathrm{M}+\mathrm{H})^{+}$.

Scheme S10. Discovery Synthesis of Compound $17^{\mathrm{a}}$
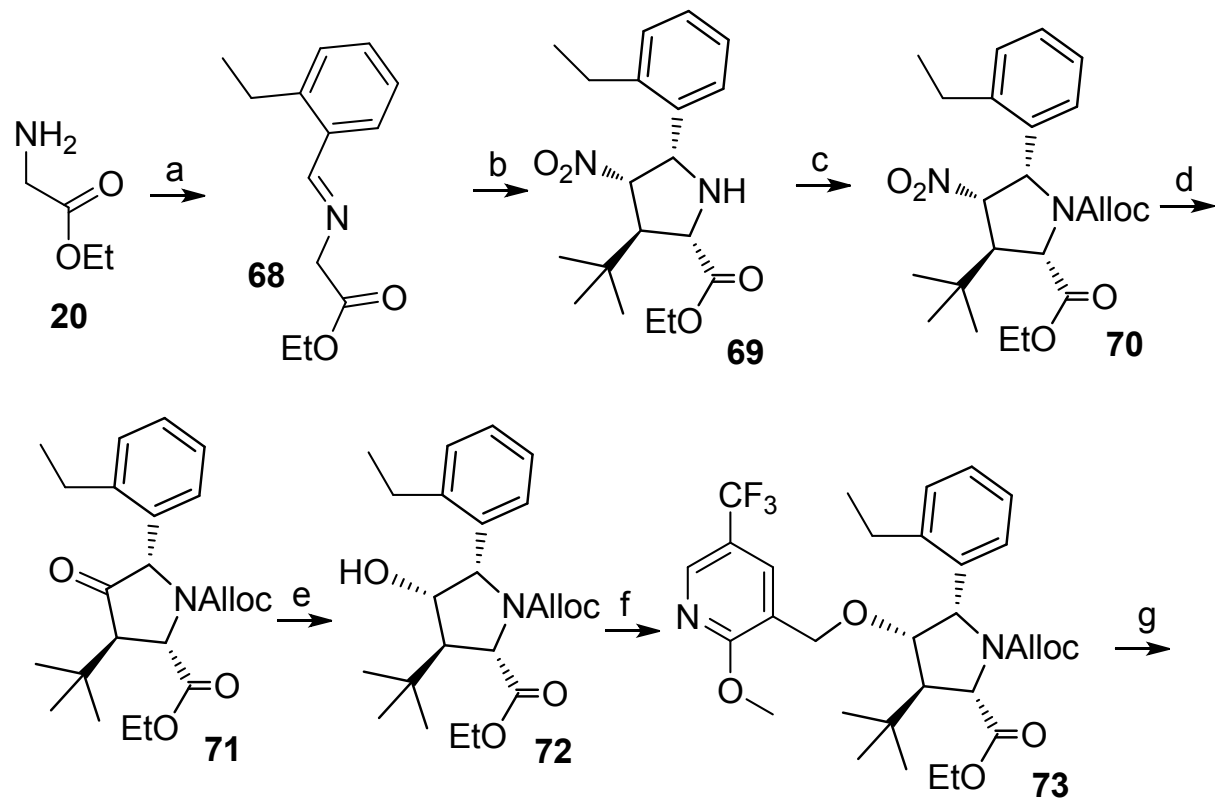<smiles>CCOC(=O)[C@H]1N[C@@H](c2ccccc2CC)[C@H](OCc2cc(C(F)(F)F)cnc2OC)[C@@H]1C(C)(C)C</smiles><smiles>CCOC(=O)[C@H]1[C@@H](C(C)(C)C)[C@@H](OCc2cc(C(F)(F)F)cnc2OC)[C@H](c2ccccc2CC)N1C(=O)[C@H]1CCCCO1</smiles><smiles>CCc1ccccc1[C@@H]1[C@H](OCc2cc(C(F)(F)F)cnc2OC)[C@@H](C(C)(C)C)[C@@H](C(=O)O)N1C(=O)[C@H]1CCCCO1</smiles> 
aReagents and conditions: (a) $\mathrm{MgSO}_{4}, \quad \mathrm{TEA}, \quad$ 2-methylbenzaldehyde, $\quad$ DCM; $\quad$ (b) (2-(Bis(3,5bis(trifluoromethyl)phenyl)phosphino)-3-((S)-4-isopropyl-4,5-dihydrooxazol-2-yl)cyclopenta-2,4-dien-1-yl)(cyclopenta-2,4-dien1-yl)iron, $\mathrm{Cu}(\mathrm{OTf})_{2}$, KOtBu, (E)-3,3-dimethyl-1-nitrobut-1-ene, THF; (c) allyl chloroformate, toluene; (d) PDC, 6N HCl, Zn dust, (e) $\mathrm{NaBH}_{4}, \mathrm{EtOH}$; (f) KOtBu, 3-(bromomethyl)-2-methoxy-5-(trifluoromethyl)pyridine, DMF; (g) 1,3-Dimethlbarbituric acid, $\mathrm{Pd}\left(\mathrm{PPh}_{3}\right)_{4}$, EtOAc/DCM; (h) Tetrahydropyran-2-carboxylic acid, oxalyl chloride, DMF, DCM; (i) LiOH, MeOH/THF.

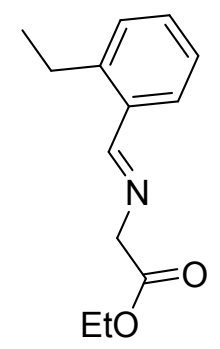

\section{(E)-ethyl 2-((2-ethylbenzylidene)amino)acetate (68)}

A mixture of ethyl 2-aminoacetate hydrochloride $(1.85 \mathrm{~g}, 13.25 \mathrm{mmol})$ and magnesium sulfate $(3.19 \mathrm{~g}$, $26.5 \mathrm{mmol})$ in dichloromethane $(22 \mathrm{~mL})$ (anhydrous) was treated with triethylamine $(1.847 \mathrm{~mL}, 13.25$ $\mathrm{mmol})$, stirred for 10 minutes and treated with 2-ethylbenzaldehyde (1.78 g, $13.25 \mathrm{mmol})$ as a solution in $1 \mathrm{~mL}$ of dichloromethane. The flask was capped and stirred at ambient temperature overnight. The solid material was filtered, the filtrate was washed with water, and the organic layer was dried with $\mathrm{Na}_{2} \mathrm{SO}_{4}$ and filtered again. The filtrate was concentrated, giving $(E)$-ethyl 2-((2-ethylbenzylidene)amino)acetate (2.65 g, 12.09 mmol, $91 \%$ yield). ${ }^{1} \mathrm{H}$ NMR (400 MHz, $\left.\mathrm{CDCl}_{3}\right) \delta \mathrm{ppm} 8.63(\mathrm{~s}, 1 \mathrm{H}), 7.96$ (dd, J = 7.8, $1.5 \mathrm{~Hz}$, 1H), 7.37 (td, J = 7.5, 1.5 Hz, 1H), $7.26(\mathrm{~d}, \mathrm{~J}=6.1 \mathrm{~Hz}, 1 \mathrm{H}), 7.25-7.19(\mathrm{~m}, 1 \mathrm{H}), 4.43(\mathrm{~d}, \mathrm{~J}=1.4 \mathrm{~Hz}, 2 \mathrm{H})$, $4.26(\mathrm{q}, \mathrm{J}=7.1 \mathrm{~Hz}, 2 \mathrm{H}), 2.89$ (q, J = 7.5 Hz, 2H), $1.32(\mathrm{t}, \mathrm{J}=7.1 \mathrm{~Hz}, 3 \mathrm{H}), 1.26(\mathrm{t}, \mathrm{J}=7.6 \mathrm{~Hz}, 3 \mathrm{H})$.

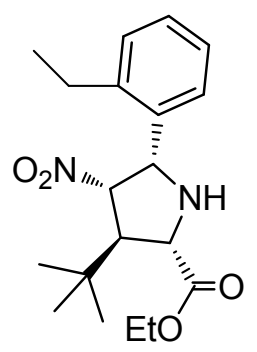

(2S,3R,4S,5S)-ethyl 3-(tert-butyl)-5-(2-ethylphenyl)-4-nitropyrrolidine-2-carboxylate (69)

(2-(Bis(3,5-bis(trifluoromethyl)phenyl)phosphino)-3-((S)-4-isopropyl-4,5-dihydrooxazol-2-yl)cyclopenta2,4-dien-1-yl)(cyclopenta-2,4-dien-1-yl)iron (0.182 g, $0.242 \mathrm{mmol})$ and copper (I) triflate dimer, benzene complex $(0.049 \mathrm{~g}, 0.097 \mathrm{mmol})$ were dissolved in tetrahydrofuran $(18 \mathrm{~mL})$ that had been sparged with an $\mathrm{N}_{2}$ stream for 1 hour. The resulting mixture was stirred for 1 hour at ambient temperature (continued nitrogen sparge), and (E)-ethyl 2-((2-ethylbenzylidene)amino)acetate $(68,2.65 \mathrm{~g}, 12.09 \mathrm{mmol})$ in $1 \mathrm{~mL}$ 
tetrahydrofuran was added. The resulting solution was cooled to $<5^{\circ} \mathrm{C}$ in an ice-water bath. Potassium 2-methylpropan-2-olate in tetrahydrofuran $(0.218 \mathrm{~mL}, 0.218 \mathrm{mmol})$ was added dropwise, followed by addition of $(E)$-3,3-dimethyl-1-nitrobut-1-ene $(1.561 \mathrm{~g}, 12.09 \mathrm{mmol})$ in $1 \mathrm{~mL}$ tetrahydrofuran over 2 minutes, maintaining a temperature less than $7^{\circ} \mathrm{C}$. The reaction mixture was stirred for 1.5 hours at $0{ }^{\circ} \mathrm{C}$. The mixture was quenched with $20 \mathrm{~mL}$ of saturated aqueous ammonium chloride and $50 \mathrm{~mL}$ of ethyl acetate and warmed to ambient temperature. The organic layer was separated and washed with saturated aqueous ammonium chloride $(2 \times 50 \mathrm{~mL})$ and brine and filtered through a pad of silica gel. The filtrate was concentrated. The crude material was purified by chromatography using a $40 \mathrm{~g}$ silica gel cartridge eluting with a gradient of $0-60 \%$ heptanes/ethyl acetate over a period of 20 minutes. The crude material was triturated with heptane and the precipitate was filtered to provide ( $2 S, 3 R, 4 S, 5 S)$-ethyl 3-(tert-butyl)5-(2-ethylphenyl)-4-nitropyrrolidine-2-carboxylate (2.11 g, $6.06 \mathrm{mmol}, 50.1 \%$ yield). ${ }^{1} \mathrm{H}$ NMR (501 MHz, DMSO-d $\left.d_{6}\right) \delta$ ppm 7.40 - $7.29(\mathrm{~m}, 1 \mathrm{H}), 7.25$ - $7.15(\mathrm{~m}, 2 \mathrm{H}), 7.11(\mathrm{td}, \mathrm{J}=7.4,1.8 \mathrm{~Hz}, 1 \mathrm{H}), 5.17(\mathrm{dd}$, $\mathrm{J}=7.0,3.5 \mathrm{~Hz}, 1 \mathrm{H}), 4.64(\mathrm{t}, \mathrm{J}=7.4 \mathrm{~Hz}, 1 \mathrm{H}), 4.19(\mathrm{qd}, \mathrm{J}=7.1,3.8 \mathrm{~Hz}, 2 \mathrm{H}), 3.72(\mathrm{t}, \mathrm{J}=7.6 \mathrm{~Hz}, 1 \mathrm{H}), 3.47$ $(\mathrm{t}, \mathrm{J}=7.5 \mathrm{~Hz}, 1 \mathrm{H}), 3.07(\mathrm{dd}, \mathrm{J}=7.9,3.5 \mathrm{~Hz}, 1 \mathrm{H}), 2.72(\mathrm{dt}, \mathrm{J}=15.0,7.5 \mathrm{~Hz}, 1 \mathrm{H}), 2.63(\mathrm{dt}, \mathrm{J}=15.0,7.5$ $\mathrm{Hz}, 1 \mathrm{H}), 1.22(\mathrm{dt}, \mathrm{J}=16.4,7.3 \mathrm{~Hz}, 6 \mathrm{H}), 0.93(\mathrm{~s}, 9 \mathrm{H})$; MS (APCI+) $m / z 349(\mathrm{M}+\mathrm{H})^{+}$.

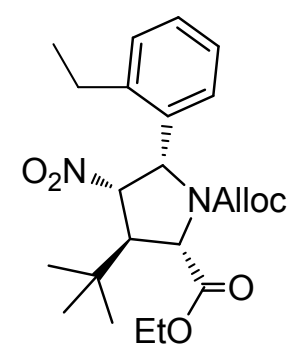

(2S,3R,4S,5S)-1-allyl 2-ethyl 3-(tert-butyl)-5-(2-ethylphenyl)-4-nitropyrrolidine-1,2-dicarboxylate (70)

To compound $69(1.43 \mathrm{~g}, 4.10 \mathrm{mmol})$ in toluene $(4.77 \mathrm{~mL})$ and saturated aqueous $\mathrm{NaHCO}_{3}(4.77 \mathrm{~mL})$ was added allyl carbonochloridate $(0.463 \mathrm{~mL}, 4.23 \mathrm{mmol})$ dropwise at ambient temperature. The mixture was stirred at ambient temperature for 16 hours. Ethyl acetate $(30 \mathrm{~mL})$ and water $(25 \mathrm{~mL})$ were added and the organic layer was washed with brine and concentrated. The resulting material was purified on a $24 \mathrm{~g}$ silica gel cartridge eluting with a gradient of 0-65\% ethyl acetate/heptanes over a period of 20 minutes to provide (2S,3R,4S,5S)-1-allyl 2-ethyl 3-(tert-butyl)-5-(2-ethylphenyl)-4-nitropyrrolidine-1,2dicarboxylate (1.64 g, $3.79 \mathrm{mmol}, 92 \%$ yield). ${ }^{1} \mathrm{H}$ NMR (400 MHz, DMSO- $\left.d_{6}\right) \delta$ ppm $7.83(\mathrm{~d}, \mathrm{~J}=7.7$ $\mathrm{Hz}, 1 \mathrm{H}), 7.19$ - $7.13(\mathrm{~m}, 1 \mathrm{H}), 7.13-7.05(\mathrm{~m}, 2 \mathrm{H}), 5.72(\mathrm{bs}, 1 \mathrm{H}), 5.66$ (dd, J = 8.8, 2.9 Hz, 1H), 5.58 (d, J $=8.8 \mathrm{~Hz}, 1 \mathrm{H}), 4.97(\mathrm{~d}, 2 \mathrm{H}), 4.53(\mathrm{~d}, \mathrm{~J}=3.8 \mathrm{~Hz}, 1 \mathrm{H}), 4.42(\mathrm{~s}, 2 \mathrm{H}), 4.24(\mathrm{q}, \mathrm{J}=7.1 \mathrm{~Hz}, 2 \mathrm{H}), 3.03(\mathrm{~d}, \mathrm{~J}=$ 
$3.5 \mathrm{~Hz}, 1 \mathrm{H}), 2.84(\mathrm{dq}, \mathrm{J}=14.8,7.4 \mathrm{~Hz}, 1 \mathrm{H}), 2.58(\mathrm{dq}, \mathrm{J}=15.1,7.6 \mathrm{~Hz}, 1 \mathrm{H}), 1.26(\mathrm{t}, \mathrm{J}=7.1 \mathrm{~Hz}, 3 \mathrm{H}), 1.18$ $(\mathrm{t}, \mathrm{J}=7.5 \mathrm{~Hz}, 3 \mathrm{H}), 1.00(\mathrm{~s}, 9 \mathrm{H})$; MS (APCI +$) \mathrm{m} / z 433(\mathrm{M}+\mathrm{H})^{+}$.

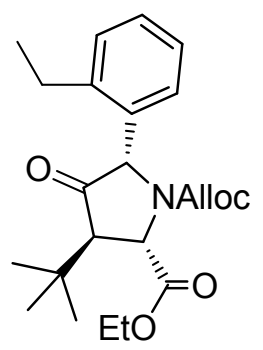

(2S,3R,5S)-1-allyl 2-ethyl 3-(tert-butyl)-5-(2-ethylphenyl)-4-oxopyrrolidine-1,2-dicarboxylate (71)

To potassium dichromate $(6.02 \mathrm{~g}, 20.48 \mathrm{mmol})$ in $6 \mathrm{~N}$ aqueous $\mathrm{HCl}(93 \mathrm{~mL})$ was added zinc $(6.84 \mathrm{~g}, 105$ mmol) portionwise under $\mathrm{N}_{2}$ atmosphere at room temperature. After the almost complete dissolution of zinc ( $\sim 2$ hours) provided a clear light blue solution, the formed chromium(II) chloride was transferred via cannula to the refluxing solution of compound $70(1.64 \mathrm{~g}, 3.79 \mathrm{mmol})$ in ethanol $(79 \mathrm{~mL})$ under $\mathrm{N}_{2}$. The reaction mixture was refluxed at $86^{\circ} \mathrm{C}$ internally for 20 hours, cooled, and concentrated. The mixture was extracted with dichloromethane three times. The organic phase was washed with saturated aqueous $\mathrm{NaHCO}_{3}$ solution and brine, dried over $\mathrm{MgSO}_{4}$, filtered, and concentrated. The residue (1.65 g) was subjected to re-esterification. Acetyl chloride $(2 \mathrm{~mL}, 28.13 \mathrm{mmol})$ was added slowly to an ice-cooled flask containing ethanol $(6 \mathrm{~mL})$. After the addition was complete, the reaction was stirred at room temperature for 5 minutes before pouring the resulting $\mathrm{HCl} /$ ethanol solution into a separate flask containing the crude ester/acid mixture. The mixture was heated to $65{ }^{\circ} \mathrm{C}$ for an additional hour, at which point nearly complete conversion was noted. The mixture was cooled to room temperature and concentrated. The crude material was purified on a $24 \mathrm{~g}$ cartridge eluting with a gradient of $0-70 \%$ ethyl acetate/heptanes over a period of 20 minutes to provide (2S,3R,5S)-1-allyl 2-ethyl 3-(tert-butyl)-5-(2ethylphenyl)-4-oxopyrrolidine-1,2-dicarboxylate (0.802 g, $1.998 \mathrm{mmol}, 52.7 \%$ yield). ${ }^{1} \mathrm{H}$ NMR (400 MHz, DMSO-d $\left.{ }_{6}\right) \delta$ ppm $7.77(\mathrm{~d}, \mathrm{~J}=7.6 \mathrm{~Hz}, 1 \mathrm{H}), 7.25$ - $7.05(\mathrm{~m}, 3 \mathrm{H}), 5.69(\mathrm{~d}, \mathrm{~J}=101.2 \mathrm{~Hz}, 1 \mathrm{H}), 5.26(\mathrm{~s}$, $1 \mathrm{H}), 4.90(\mathrm{~d}, 1 \mathrm{H}), 4.73-4.29(\mathrm{~m}, 4 \mathrm{H}), 4.20(\mathrm{qd}, \mathrm{J}=7.1,1.6 \mathrm{~Hz}, 2 \mathrm{H}), 2.73(\mathrm{tt}, \mathrm{J}=14.5,7.1 \mathrm{~Hz}, 3 \mathrm{H}), 1.24$ $(\mathrm{t}, \mathrm{J}=7.1 \mathrm{~Hz}, 3 \mathrm{H}), 1.16(\mathrm{t}, \mathrm{J}=7.5 \mathrm{~Hz}, 3 \mathrm{H}), 1.03(\mathrm{~s}, 9 \mathrm{H}) ; \mathrm{MS}(\mathrm{APCI}+) \mathrm{m} / z 402(\mathrm{M}+\mathrm{H})^{+}$.

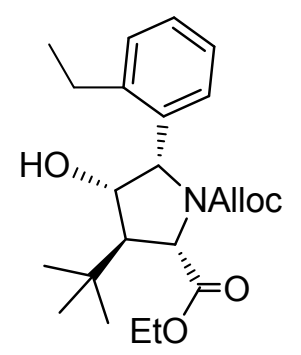




\section{$(2 S, 3 R, 4 S, 5 S)$-1-allyl 2-ethyl 3-(tert-butyl)-5-(2-ethylphenyl)-4-hydroxypyrrolidine-1,2-}

dicarboxylate (72)

Compound $71(0.8 \mathrm{~g}, 1.993 \mathrm{mmol})$ was dissolved in ethanol $(9.96 \mathrm{~mL})$ and sodium borohydride $(0.151 \mathrm{~g}$, $3.99 \mathrm{mmol}$ ) was added after cooling the reaction to $<-10{ }^{\circ} \mathrm{C}$ in an ice/acetone bath. The ice bath was removed and the mixture was allowed to warm to room temperature over 20 minutes. The reaction mixture was concentrated and partitioned between ethyl acetate and saturated aqueous sodium bicarbonate. The organics were concentrated and purified using a $40 \mathrm{~g}$ silica gel cartridge and eluting with $0-2 \%$ methanol/dichloromethane over a period of 20 minutes to provide $(2 S, 3 R, 4 S, 5 S)$-1-allyl 2ethyl 3-(tert-butyl)-5-(2-ethylphenyl)-4-hydroxypyrrolidine-1,2-dicarboxylate, 2173 (601 mg, 1.489 mmol, $74.7 \%$ yield). ${ }^{1} \mathrm{H}$ NMR (400 MHz, DMSO- $\left.d_{6}\right) \delta$ ppm $7.92(\mathrm{~s}, 1 \mathrm{H}), 7.13$ - $7.02(\mathrm{~m}, 3 \mathrm{H}), 5.67(\mathrm{~d}, \mathrm{~J}$ $=107.5 \mathrm{~Hz}, 1 \mathrm{H}), 5.23(\mathrm{~m}, 1 \mathrm{H}), 5.07(\mathrm{~d}, \mathrm{~J}=6.6 \mathrm{~Hz}, 1 \mathrm{H}), 4.85(\mathrm{~m}, 1 \mathrm{H}), 4.61(\mathrm{~s}, 1 \mathrm{H}), 4.47$ - $4.27(\mathrm{~m}, 3 \mathrm{H})$, $4.24(\mathrm{~d}, \mathrm{~J}=4.9 \mathrm{~Hz}, 1 \mathrm{H}), 4.14(\mathrm{qd}, \mathrm{J}=7.1,1.9 \mathrm{~Hz}, 2 \mathrm{H}), 2.69(\mathrm{dq}, \mathrm{J}=14.4,7.2 \mathrm{~Hz}, 1 \mathrm{H}), 2.54(\mathrm{dd}, \mathrm{J}=14.7$, $7.5 \mathrm{~Hz}, 1 \mathrm{H}), 2.23(\mathrm{~s}, 1 \mathrm{H}), 1.22(\mathrm{t}, \mathrm{J}=7.1 \mathrm{~Hz}, 3 \mathrm{H}), 1.16(\mathrm{t}, \mathrm{J}=7.4 \mathrm{~Hz}, 3 \mathrm{H}), 0.95(\mathrm{~s}, 9 \mathrm{H})$; MS (APCI+) $m / z$ $404(\mathrm{M}+\mathrm{H})^{+}$.

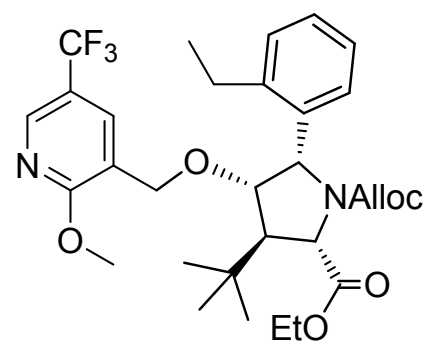

\section{(2S,3R,4S,5S)-1-allyl 2-ethyl 3-(tert-butyl)-5-(2-ethylphenyl)-4-((2-methoxy-5-}

\section{(trifluoromethyl)pyridin-3-yl)methoxy)pyrrolidine-1,2-dicarboxylate (73)}

Compound 72 (400 mg, $0.991 \mathrm{mmol}$ ) and 3-(bromomethyl)-2-methoxy-5-(trifluoromethyl)pyridine (428 $\mathrm{mg}, 1.586 \mathrm{mmol})$ were dissolved in dry dimethylformamide $(5 \mathrm{~mL})$. After cooling in an ice bath, potassium 2-methylpropan-2-olate $(1.437 \mathrm{~mL}, 1.437 \mathrm{mmol})$ solution was added dropwise over 4 minutes. After 60 minutes, the reaction was acidified with $1 \mathrm{M}$ aqueous $\mathrm{HCl}(1.2 \mathrm{~mL})$ and warmed to room temperature. The reaction mixture was diluted with water $(20 \mathrm{~mL})$ and extracted with dichloromethane. The organic extracts were loaded onto a $24 \mathrm{~g}$ silica gel column and eluted with a gradient of $0-80 \%$ ethyl acetate/heptanes over 20 minutes to provide (2S,3R,4S,5S)-1-allyl 2-ethyl 3-(tert-butyl)-5-(2ethylphenyl)-4-((2-methoxy-5-(trifluoromethyl)pyridin-3-yl)methoxy)pyrrolidine-1,2-dicarboxylate (421 $\mathrm{mg}, 0.710 \mathrm{mmol}, 71.7 \%$ yield). ${ }^{1} \mathrm{H}$ NMR (400 MHz, DMSO- $\left.d_{6}\right) \delta \mathrm{ppm} 8.33$ (dd, J = 2.5, $1.2 \mathrm{~Hz}, 1 \mathrm{H}$ ), $7.91(\mathrm{~s}, 1 \mathrm{H}), 7.15$ - $6.93(\mathrm{~m}, 4 \mathrm{H}), 5.66(\mathrm{~d}, \mathrm{~J}=128.9 \mathrm{~Hz}, 1 \mathrm{H}), 5.18(\mathrm{~d}, \mathrm{~J}=5.4 \mathrm{~Hz}, 3 \mathrm{H}), 5.00$ - 4.53 (m, 1H), $4.47(\mathrm{~d}, \mathrm{~J}=1.8 \mathrm{~Hz}, 2 \mathrm{H}), 4.31(\mathrm{~s}, 2 \mathrm{H}), 4.28(\mathrm{~d}, \mathrm{~J}=14.6 \mathrm{~Hz}, 2 \mathrm{H}), 4.06(\mathrm{qt}, \mathrm{J}=7.1,3.6 \mathrm{~Hz}, 2 \mathrm{H}), 3.82(\mathrm{~d}, \mathrm{~J}=$ 
14.6 Hz, 4H), 2.73 (dq, J = 14.9, 7.4 Hz, 1H), 2.54 (dt, J = 14.7, $7.5 \mathrm{~Hz}, 1 \mathrm{H}), 1.14$ (t, J = 7.6 Hz, 3H), $1.08(\mathrm{t}, \mathrm{J}=7.1 \mathrm{~Hz}, 3 \mathrm{H}), 1.00(\mathrm{~s}, 9 \mathrm{H})$; MS (APCI+) $m / z 593(\mathrm{M}+\mathrm{H})^{+}$.

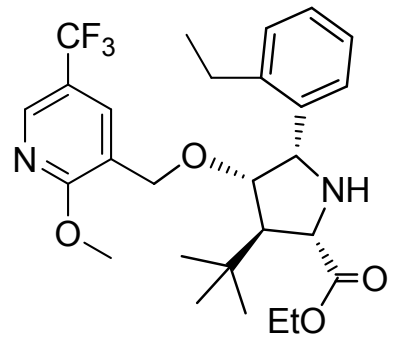

(2S,3R,4S,5S)-ethyl 3-(tert-butyl)-5-(2-ethylphenyl)-4-((2-methoxy-5-(trifluoromethyl)pyridin-3yl)methoxy)pyrrolidine-2-carboxylate (74)

To a solution of compound 73 (420 mg, $0.709 \mathrm{mmol})$ in acetonitrile/water $(1.24 \mathrm{~mL}, 10: 1)$ was added tetrakis(triphenylphosphine)palladium $(0)(18.02 \mathrm{mg}, 0.016 \mathrm{mmol})$ and diethylamine $(0.147 \mathrm{~mL}, 1.417$ mmol) The mixture was stirred at room temperature overnight. Dichloromethane and water were added, and the organic layer was washed with brine, dried over $\mathrm{MgSO}_{4}$, filtered, and concentrated. The crude material was purified by chromatography, eluting on $12 \mathrm{~g}$ silica gel cartridge with a gradient of $0-70 \%$ ethyl acetate/heptanes over 20 minutes to provide (2S,3R,4S,5S)-ethyl 3-(tert-butyl)-5-(2-ethylphenyl)-4((2-methoxy-5-(trifluoromethyl)pyridin-3-yl)methoxy)pyrrolidine-2-carboxylate (321 mg, 0.631 mmol, $89 \%$ yield). ${ }^{1} \mathrm{H}$ NMR (501 MHz, DMSO- $\left.d_{6}\right) \delta$ ppm $8.41-8.30(\mathrm{~s}, 1 \mathrm{H}), 7.67-7.55(\mathrm{~d}, 1 \mathrm{H}), 7.27(\mathrm{~d}, \mathrm{~J}=$ $2.5 \mathrm{~Hz}, 1 \mathrm{H}), 7.19$ - 7.09 (m, 2H), $7.05(\mathrm{ddd}, \mathrm{J}=8.5,5.7,3.0 \mathrm{~Hz}, 1 \mathrm{H}), 4.25(\mathrm{~d}, \mathrm{~J}=4.5 \mathrm{~Hz}, 1 \mathrm{H}), 4.22(\mathrm{~d}, \mathrm{~J}=$ $13.8 \mathrm{~Hz}, 2 \mathrm{H}), 4.17$ - 4.07 (m, 2H), $3.96(\mathrm{dd}, \mathrm{J}=4.5,1.6 \mathrm{~Hz}, 1 \mathrm{H}), 3.83(\mathrm{~s}, 3 \mathrm{H}), 3.72(\mathrm{~d}, \mathrm{~J}=13.9 \mathrm{~Hz}, 1 \mathrm{H})$, 3.59 (t, J = 7.1 Hz, 1H), 3.12 (t, J = 7.8 Hz, 1H), 2.68 (dq, J = 15.0, $7.5 \mathrm{~Hz}, 1 \mathrm{H}), 2.59$ (dq, J = 14.9, 7.5 $\mathrm{Hz}, 1 \mathrm{H}), 2.34(\mathrm{dd}, \mathrm{J}=6.8,1.5 \mathrm{~Hz}, 1 \mathrm{H}), 1.22$ - 1.17 (t, 3H), 1.17 - $1.13(\mathrm{t}, 3 \mathrm{H}), 0.95$ (s, 9H); MS (APCI+) $m / z 509(\mathrm{M}+\mathrm{H})^{+}$.

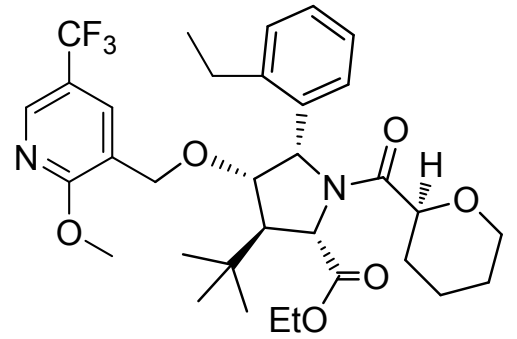

(2S,3R,4S,5S)-ethyl 3-(tert-butyl)-5-(2-ethylphenyl)-4-((2-methoxy-5-(trifluoromethyl)pyridin-3yl)methoxy)-1-((S)-tetrahydro-2H-pyran-2-carbonyl)pyrrolidine-2-carboxylate (75)

To a solution of compound $74(320 \mathrm{mg}, 0.629 \mathrm{mmol})$ in dichloromethane $(2.5 \mathrm{~mL})$ at $0^{\circ} \mathrm{C}$ was added triethylamine $(0.395 \mathrm{~mL}, 2.83 \mathrm{mmol})$ followed by tetrahydro-2H-pyran-2-carbonyl chloride $(159 \mathrm{mg}$, 
$1.070 \mathrm{mmol}$ ) as a solution in $2 \mathrm{~mL}$ dichloromethane. After stirring for 15 minutes, the reaction was quenched with $5 \mathrm{~mL}$ of saturated aqueous sodium bicarbonate. The crude material was chromatographed three times using a $24 \mathrm{~g}$ silica gel cartridge (3 times with a gradient of 0-80\% ethyl acetate/heptanes over a period of 20 minutes to provide $(2 S, 3 R, 4 S, 5 S)$-ethyl 3-(tert-butyl)-5-(2-ethylphenyl)-4-((2-methoxy-5(trifluoromethyl)pyridin-3-yl)methoxy)-1-((S)-tetrahydro-2H-pyran-2-carbonyl)pyrrolidine-2-carboxylate (166 mg, $0.267 \mathrm{mmol}, 42.5 \%$ yield). ${ }^{1} \mathrm{H}$ NMR (400 MHz, DMSO- $\left.d_{6}\right) \delta \mathrm{ppm} 8.27$ (d, J = 2.4 Hz, 1H), 8.02 - $7.94(\mathrm{~d}, 1 \mathrm{H}), 7.20$ (s, 1H), 7.13 (s, 2H), 7.04 (d, J = $7.5 \mathrm{~Hz}, 1 \mathrm{H}), 5.56(\mathrm{~s}, 1 \mathrm{H}), 4.69$ (s, 1H), 4.32 $4.20(\mathrm{~m}, 2 \mathrm{H}), 4.09(\mathrm{qd}, \mathrm{J}=7.0,3.0 \mathrm{~Hz}, 2 \mathrm{H}), 3.85(\mathrm{~s}, 3 \mathrm{H}), 3.80(\mathrm{~d}, \mathrm{~J}=13.8 \mathrm{~Hz}, 1 \mathrm{H}), 3.72$ (d, J = 11.4 Hz, 1H), 3.41 (bs, 2H), 2.75 (dt, J = 15.1, 7.6 Hz, 1H), 2.66 (dd, J = 14.9, 7.4 Hz, 1H), 2.42 (d, J = 1.8 Hz, $1 \mathrm{H}), 1.65(\mathrm{~d}, \mathrm{~J}=12.9 \mathrm{~Hz}, 1 \mathrm{H}), 1.52(\mathrm{~d}, \mathrm{~J}=11.9 \mathrm{~Hz}, 2 \mathrm{H}), 1.34(\mathrm{~s}, 3 \mathrm{H}), 1.22(\mathrm{t}, \mathrm{J}=7.5 \mathrm{~Hz}, 3 \mathrm{H}), 1.14(\mathrm{t}, \mathrm{J}=$ $7.1 \mathrm{~Hz}, 3 \mathrm{H}), 1.03(\mathrm{~s}, 9 \mathrm{H})$; MS (APCI+) $\mathrm{m} / z 621(\mathrm{M}+\mathrm{H})^{+}$. Also obtained was $(2 S, 3 R, 4 S, 5 S)$-ethyl 3-(tertbutyl)-5-(2-ethylphenyl)-4-((2-methoxy-5-(trifluoromethyl)pyridin-3-yl)methoxy)-1-((R)-tetrahydro-2Hpyran-2-carbonyl)pyrrolidine-2-carboxylate (168 mg, $0.271 \mathrm{mmol}, 43.0 \%$ yield). ${ }^{1} \mathrm{H}$ NMR (400 MHz, DMSO- $\left.d_{6}\right) \delta$ ppm $8.27(\mathrm{~s}, 1 \mathrm{H}), 7.78(\mathrm{~s}, 1 \mathrm{H}), 7.15(\mathrm{~d}, \mathrm{~J}=2.4 \mathrm{~Hz}, 1 \mathrm{H}), 7.09-7.03(\mathrm{~m}, 2 \mathrm{H}), 6.97(\mathrm{~d}, \mathrm{~J}=8.3$ $\mathrm{Hz}, 1 \mathrm{H}), 5.31(\mathrm{~d}, \mathrm{~J}=5.6 \mathrm{~Hz}, 1 \mathrm{H}), 4.94(\mathrm{~s}, 1 \mathrm{H}), 4.29$ - $4.20(\mathrm{~m}, 2 \mathrm{H}), 4.13(\mathrm{qd}, \mathrm{J}=7.1,2.2 \mathrm{~Hz}, 2 \mathrm{H}), 3.91$ (bs, 2H), $3.85(\mathrm{~s}, 3 \mathrm{H}), 3.83-3.76(\mathrm{~m}, 1 \mathrm{H}), 3.26(\mathrm{bs}, 1 \mathrm{H}), 2.75(\mathrm{dq}, \mathrm{J}=14.9,7.6 \mathrm{~Hz}, 1 \mathrm{H}), 2.63(\mathrm{dt}, \mathrm{J}=$ 14.8, 7.5 Hz, 1H), $2.57(\mathrm{~d}, \mathrm{~J}=2.4 \mathrm{~Hz}, 1 \mathrm{H}), 1.77(\mathrm{~s}, 1 \mathrm{H}), 1.51(\mathrm{~s}, 2 \mathrm{H}), 1.41(\mathrm{~s}, 3 \mathrm{H}), 1.22$ (t, J = 7.5 Hz, $3 \mathrm{H}), 1.15(\mathrm{t}, \mathrm{J}=7.1 \mathrm{~Hz}, 3 \mathrm{H}), 1.03(\mathrm{~s}, 9 \mathrm{H})$; MS (APCI+) $m / z 621(\mathrm{M}+\mathrm{H})^{+}$.

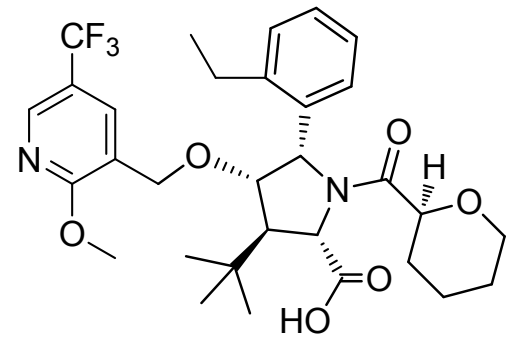

(2S,3R,4S,5S)-3-tert-butyl-5-(2-ethylphenyl)-4-\{[2-methoxy-5-(trifluoromethyl)pyridin-3yl]methoxy\}-1-[(2S)-oxane-2-carbonyl]pyrrolidine-2-carboxylic acid (17)

To a solution of compound $75(156 \mathrm{mg}, 0.251 \mathrm{mmol})$ in tetrahydrofuran $(0.760 \mathrm{~mL})$, methanol $(0.760$ $\mathrm{mL})$, and water $(0.760 \mathrm{~mL})$ was added lithium hydroxide, $\mathrm{H}_{2} \mathrm{O}(73.8 \mathrm{mg}, 1.759 \mathrm{mmol})$. The reaction mixture was heated at $45^{\circ} \mathrm{C}$ for 16 hours. LC/MS showed desired product and the solvent was removed under a stream of nitrogen. Water $(0.5 \mathrm{~mL})$ was added to the crude material. The mixture was acidified with $1 \mathrm{M}$ aqueous $\mathrm{HCl}(1.53 \mathrm{~mL})$ to $\mathrm{pH} \sim 6$, extracted once with heptanes (discarded), and once with dichloromethane. The solvent was evaporated in vacuo. The resulted crude material was chromatographed using a $4 \mathrm{~g}$ silica gel cartridge with a gradient of $0-10 \%$ methanol/dichloromethane over 
a period of 10 minutes to provide (2S,3R,4S,5S)-3-(tert-butyl)-5-(2-ethylphenyl)-4-((2-methoxy-5-

(trifluoromethyl)pyridin-3-yl)methoxy)-1-((S)-tetrahydro-2H-pyran-2-carbonyl)pyrrolidine-2-carboxylic acid (87 mg, $0.147 \mathrm{mmol}, 58.4 \%$ yield). ${ }^{1} \mathrm{H}$ NMR (400 MHz, DMSO- $\left.d_{6}\right) \delta$ ppm $8.26(\mathrm{~s}, 1 \mathrm{H}), 8.05$ (d, J $=7.3 \mathrm{~Hz}, 1 \mathrm{H}), 7.16(\mathrm{~s}, 1 \mathrm{H}), 7.10(\mathrm{~s}, 2 \mathrm{H}), 7.03(\mathrm{~m}, 1 \mathrm{H}), 5.56(\mathrm{~s}, 1 \mathrm{H}), 4.65(\mathrm{~s}, 1 \mathrm{H}), 4.28(\mathrm{~d}, \mathrm{~J}=14.0 \mathrm{~Hz}$, $1 \mathrm{H}), 4.23(\mathrm{~d}, \mathrm{~J}=5.8 \mathrm{~Hz}, 1 \mathrm{H}), 3.86(\mathrm{~d}, 4 \mathrm{H}), 3.73(\mathrm{~d}, \mathrm{~J}=11.5 \mathrm{~Hz}, 1 \mathrm{H}), 3.42$ (bs, 2H), 2.79 - 2.69 (m, 1H), $2.64(\mathrm{dq}, \mathrm{J}=15.0,7.6 \mathrm{~Hz}, 1 \mathrm{H}), 2.48(\mathrm{~m}, 1 \mathrm{H}), 1.71-1.60(\mathrm{~m}, 1 \mathrm{H}), 1.52(\mathrm{~m}, 2 \mathrm{H}), 1.34(\mathrm{~m}, 3 \mathrm{H}), 1.21(\mathrm{t}, \mathrm{J}=$ $7.5 \mathrm{~Hz}, 3 \mathrm{H}), 1.02(\mathrm{~s}, 9 \mathrm{H})$; MS (APCI+) $m / z 593(\mathrm{M}+\mathrm{H})^{+}$.

\section{Scheme S11. Discovery Synthesis of Compound $1^{\mathrm{a}}$}
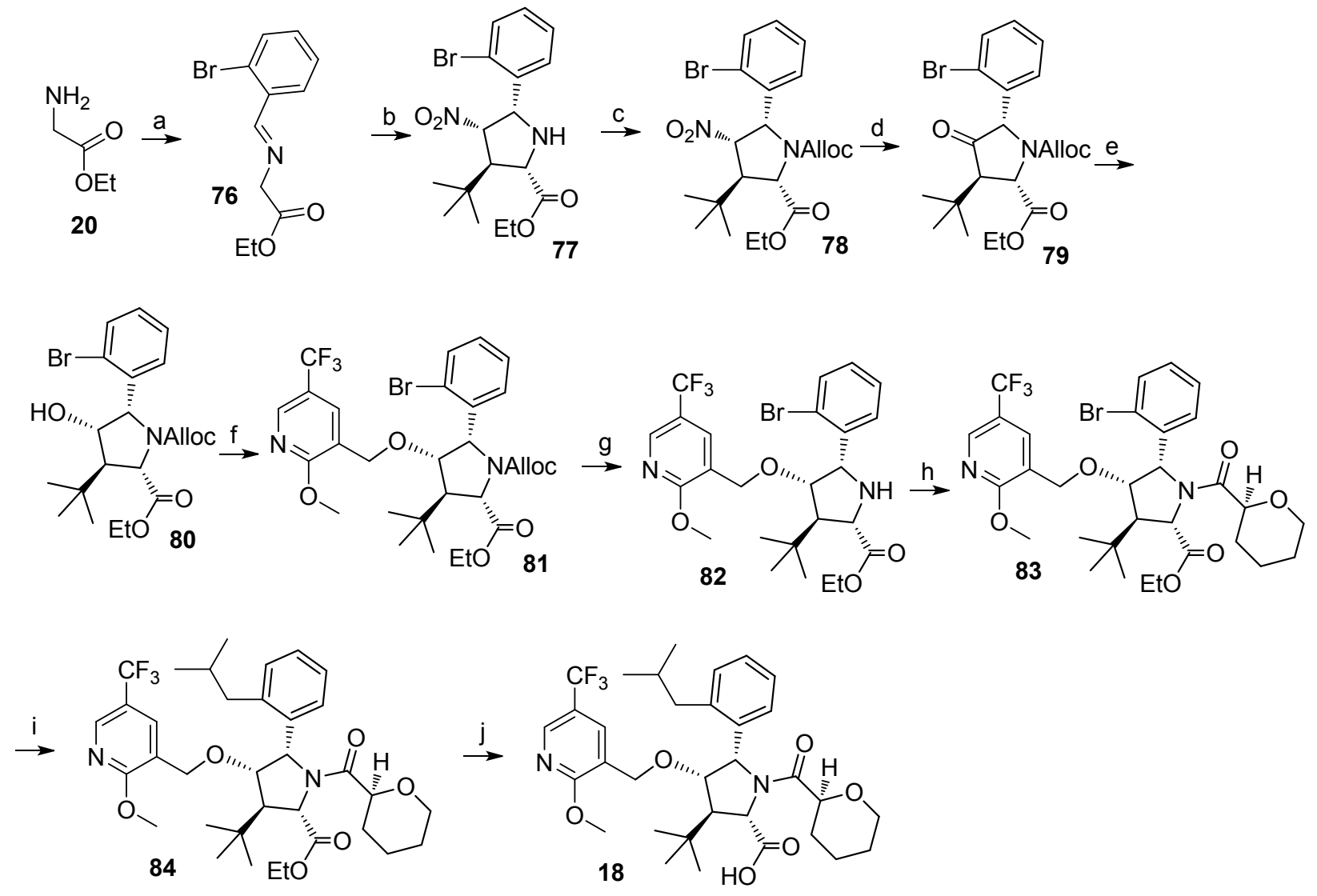

aReagents and conditions: (a) $\mathrm{MgSO}_{4}, \quad \mathrm{TEA}, \quad$ 2-methylbenzaldehyde, DCM; $\quad$ (b) (2-(Bis(3,5bis(trifluoromethyl)phenyl)phosphino)-3-((S)-4-isopropyl-4,5-dihydrooxazol-2-yl)cyclopenta-2,4-dien-1-yl)(cyclopenta-2,4-dien1-yl)iron, $\mathrm{Cu}(\mathrm{OTf})_{2}$, KOtBu, (E)-3,3-dimethyl-1-nitrobut-1-ene, THF; (c) allyl chloroformate, toluene; (d) PDC, 6N HCl, Zn dust, (e) $\mathrm{NaBH}_{4}$, EtOH; (f) KOtBu, 3-(bromomethyl)-2-methoxy-5-(trifluoromethyl)pyridine, DMF; (g) 1,3-Dimethlbarbituric acid, $\mathrm{Pd}\left(\mathrm{PPh}_{3}\right)_{4}$, EtOAc/DCM; (h) Tetrahydropyran-2-carboxylic acid, oxalyl chloride, DMF, DCM; (i) isobutylboronic acid, $\mathrm{K}_{2} \mathrm{CO}_{3}, \mathrm{Pd}(\mathrm{dppf}) \mathrm{Cl}_{2} ;$ (j) LiOH, MeOH/THF. 


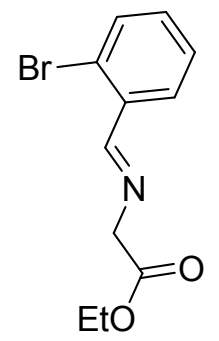

\section{(E)-ethyl 2-((2-bromobenzylidene)amino)acetate (76)}

Ethyl 2-aminoacetate hydrochloride $(2.63 \mathrm{~g}, 18.85 \mathrm{mmol})$ and magnesium sulfate $(2.269 \mathrm{~g}, 18.85 \mathrm{mmol})$ were stirred in dichloromethane $(28.6 \mathrm{~mL})$ at ambient temperature, and triethylamine $(2.63 \mathrm{~mL}, 18.85$ mmol) was added. The mixture was stirred for 5 minutes, 2-bromobenzaldehyde $(2.0 \mathrm{~mL}, 17.13 \mathrm{mmol})$ was added dropwise, and the mixture was stirred at ambient temperature for 16 hours. The solid material was filtered through a disposable plastic frit and washed with dichloromethane. The organic layer was washed with $30 \mathrm{~mL}$ of water then dried over sodium sulfate, filtered, and concentrated to provide $(E)$ ethyl 2-((2-bromobenzylidene)amino)acetate (4.6 g, $17.03 \mathrm{mmol}, 99 \%$ yield). ${ }^{1} \mathrm{H} \mathrm{NMR}(400 \mathrm{MHz}$, $\left.\mathrm{CDCl}_{3}\right) \delta \mathrm{ppm} 8.70(\mathrm{~d}, \mathrm{~J}=1.6 \mathrm{~Hz}, 1 \mathrm{H}), 8.12(\mathrm{dd}, \mathrm{J}=7.7,1.9 \mathrm{~Hz}, 1 \mathrm{H}), 7.60(\mathrm{dd}, \mathrm{J}=7.8,1.3 \mathrm{~Hz}, 1 \mathrm{H}), 7.38$ $(\mathrm{tt}, \mathrm{J}=7.6,1.1 \mathrm{~Hz}, 1 \mathrm{H}), 7.35-7.27(\mathrm{~m}, 1 \mathrm{H}), 4.48(\mathrm{~d}, \mathrm{~J}=1.4 \mathrm{~Hz}, 2 \mathrm{H}), 4.28(\mathrm{q}, \mathrm{J}=7.1 \mathrm{~Hz}, 2 \mathrm{H}), 1.34(\mathrm{t}, \mathrm{J}=$ $7.1 \mathrm{~Hz}, 3 \mathrm{H})$; MS (ESI+) $m / z 270(\mathrm{M}+\mathrm{H})^{+}$.

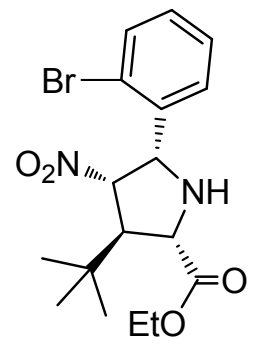

(2S,3R,4S,5S)-ethyl 5-(2-bromophenyl)-3-(tert-butyl)-4-nitropyrrolidine-2-carboxylate (77) (2-(Bis(3,5-bis(trifluoromethyl)phenyl)phosphino)-3-((S)-4-isopropyl-4,5-dihydrooxazol-2-yl)cyclopenta2,4-dien-1-yl)(cyclopenta-2,4-dien-1-yl)iron (0.147 g, $0.195 \mathrm{mmol})$ and copper (I) triflate dimer, benzene complex $(0.042 \mathrm{~g}, 0.084 \mathrm{mmol})$ were dissolved in tetrahydrofuran $(34.3 \mathrm{~mL})$ that had been sparged with stream of nitrogen for 1 hour. The resulting mixture was stirred for 1.5 hours at ambient temperature, and (E)-ethyl 2-((2-bromobenzylidene)amino)acetate (76, $4.63 \mathrm{~g}, 17.14 \mathrm{mmol})$ was added after cooling to $<5$ ${ }^{\circ} \mathrm{C}$ in an ice-water bath. Potassium 2-methylpropan-2-olate $(0.134 \mathrm{~mL}, 0.134 \mathrm{mmol})$ was added dropwise, followed by addition of (E)-3,3-dimethyl-1-nitrobut-1-ene (2.324 g, $18.00 \mathrm{mmol})$ neat over 25 minutes, maintaining an internal temperature $<10^{\circ} \mathrm{C}$. After the addition was complete and stirred for 90 minutes, LC-MS showed complete conversion. The mixture was diluted with methyl tert-butyl ether (150 $\mathrm{mL}$ ) and stirred with $50 \mathrm{~mL}$ of saturated aqueous ammonium chloride at ambient temperature for 15 
minutes. The layers were separated and the organic layer was washed with saturated aqueous sodium bicarbonate and brine. The organic layer was dried over sodium sulfate, filtered, and concentrated, and precipitated from $50 \mathrm{~mL}$ of heptane. The mixture was cooled in an ice bath to $<5^{\circ} \mathrm{C}$ for 15 minutes, and the resulting material was filtered and washed with $20 \mathrm{~mL}$ of heptanes to provide $(2 S, 3 R, 4 S, 5 S)$-ethyl 5 (2-bromophenyl)-3-(tert-butyl)-4-nitropyrrolidine-2-carboxylate (4.303 g, $10.78 \mathrm{mmol}, 62.9 \%$ yield). ${ }^{1} \mathrm{H}$ NMR (400 MHz, $\left.\mathrm{CDCl}_{3}\right) \delta \mathrm{ppm} 7.58(\mathrm{dd}, \mathrm{J}=8.0,1.2 \mathrm{~Hz}, 1 \mathrm{H}), 7.41$ - $7.29(\mathrm{~m}, 2 \mathrm{H}), 7.24$ - $7.14(\mathrm{~m}$, 1H), $5.43(\mathrm{dd}, \mathrm{J}=5.9,2.3 \mathrm{~Hz}, 1 \mathrm{H}), 4.70$ (dd, $\mathrm{J}=10.7,5.9 \mathrm{~Hz}, 1 \mathrm{H}), 4.33$ (qd, J = 7.1, 1.2 Hz, 2H), 3.82 (t, $\mathrm{J}=7.5 \mathrm{~Hz}, 1 \mathrm{H}), 3.22(\mathrm{t}, \mathrm{J}=9.8 \mathrm{~Hz}, 1 \mathrm{H}), 3.03(\mathrm{dd}, \mathrm{J}=7.0,2.3 \mathrm{~Hz}, 1 \mathrm{H}), 1.36(\mathrm{t}, \mathrm{J}=7.1 \mathrm{~Hz}, 3 \mathrm{H}), 1.08(\mathrm{~s}$, 9H); MS (APCI+) $m / z 399(\mathrm{M}+\mathrm{H})^{+}$.

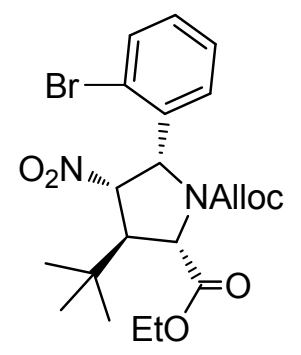

(2S,3R,4S,5S)-1-allyl 2-ethyl 5-(2-bromophenyl)-3-(tert-butyl)-4-nitropyrrolidine-1,2-dicarboxylate (78)

Compound $77(957 \mathrm{mg}, 2.397 \mathrm{mmol})$ in toluene $(10 \mathrm{~mL})$ and saturated aqueous $\mathrm{NaHCO}_{3}(10 \mathrm{~mL})$ was added allyl carbonochloridate $(0.3 \mathrm{~mL}, 2.82 \mathrm{mmol})$ dropwise at ambient temperature. The mixture was stirred at ambient temperature overnight. The reaction was poured into water $(50 \mathrm{~mL})$ and extracted with dichloromethane $(3 \times 50 \mathrm{~mL})$. The organic layer was washed with brine and concentrated and purified by silica gel chromatography using a $40 \mathrm{~g}$ cartridge and eluting with $0->5 \%$ ethyl acetate in dichloromethane to provide (2S,3R,4S,5S)-1-allyl 2-ethyl 5-(2-bromophenyl)-3-(tert-butyl)-4nitropyrrolidine-1,2-dicarboxylate (1.16 g, $2.400 \mathrm{mmol}, 100 \%$ yield). ${ }^{1} \mathrm{H}$ NMR (400 MHz, DMSO- $d_{6}$, $\left.120{ }^{\circ} \mathrm{C}\right) \delta \mathrm{ppm} 7.78(\mathrm{dd}, \mathrm{J}=7.7,1.5 \mathrm{~Hz}, 1 \mathrm{H}), 7.24-7.04(\mathrm{~m}, 2 \mathrm{H}), 6.99(\mathrm{dd}, \mathrm{J}=7.5,1.5 \mathrm{~Hz}, 1 \mathrm{H}), 6.00(\mathrm{~d}$, $\mathrm{J}=8.6 \mathrm{~Hz}, 1 \mathrm{H}), 5.67(\mathrm{ddt}, \mathrm{J}=17.2,10.5,5.2 \mathrm{~Hz}, 1 \mathrm{H}), 5.56(\mathrm{dd}, \mathrm{J}=8.6,2.5 \mathrm{~Hz}, 1 \mathrm{H}), 5.05-4.92(\mathrm{~m}, 2 \mathrm{H})$, $4.60(\mathrm{~d}, \mathrm{~J}=3.4 \mathrm{~Hz}, 1 \mathrm{H}), 4.41(\mathrm{dq}, \mathrm{J}=4.8,1.5 \mathrm{~Hz}, 2 \mathrm{H}), 4.31-4.21(\mathrm{~m}, 2 \mathrm{H}), 3.06(\mathrm{t}, \mathrm{J}=3.0 \mathrm{~Hz}, 1 \mathrm{H}), 2.06$ $(\mathrm{tt}, \mathrm{J}=8.4,5.4 \mathrm{~Hz}, 1 \mathrm{H}), 1.30(\mathrm{t}, \mathrm{J}=7.1 \mathrm{~Hz}, 3 \mathrm{H}), 1.04(\mathrm{~s}, 9 \mathrm{H}), 1.02-0.89$ (m, 2H), 0.80 (dtd, J = 9.4, 5.2, $3.4 \mathrm{~Hz}, 1 \mathrm{H}), 0.59-0.48(\mathrm{~m}, 1 \mathrm{H})$; $\mathrm{MS}(\mathrm{APCI}+) \mathrm{m} / z$ 483/485 (M+H) ${ }^{+} \mathrm{Br}$ doublet. 


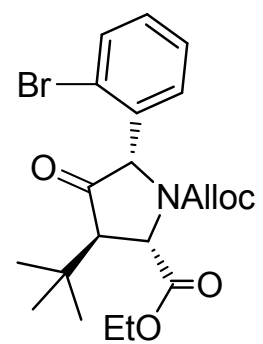

(2S,3R,5S)-1-allyl 2-ethyl 5-(2-bromophenyl)-3-(tert-butyl)-4-oxopyrrolidine-1,2-dicarboxylate (79) To a solution of potassium dichromate $(3.72 \mathrm{~g}, 12.65 \mathrm{mmol})$ in $6 \mathrm{~N}$ aqueous $\mathrm{HCl}(50 \mathrm{~mL})$ was added zinc (4.41 g, $67.5 \mathrm{mmol}$ ) under $\mathrm{N}_{2}$ atmosphere. After the almost complete dissolution of zinc yielded a clear bright blue solution, the formed chromium(II) chloride was transferred to the refluxing solution of compound 78 (1.14 g, $2.359 \mathrm{mmol})$ in ethanol $(25 \mathrm{~mL})$ under $\mathrm{N}_{2}$ using an addition funnel (internal temp $78^{\circ} \mathrm{C}$ ). The reaction mixture was refluxed at $85^{\circ} \mathrm{C}$ overnight and cooled and diluted with $300 \mathrm{~mL}$ of dichloromethane. The organic phase was separated, dried over sodium sulfate, filtered and concentrated. The crude material was then taken up in anhydrous ethanol and treated with a solution of $\mathrm{HCl}$ in ethanol (prepared by adding $2.0 \mathrm{~mL}$ of acetyl chloride to an ice bath cooled solution of $6 \mathrm{~mL}$ of ethanol), and heated at $65^{\circ} \mathrm{C}$ for one hour. The ethanol was removed and the crude material was diluted with $300 \mathrm{~mL}$ of methyl tert-butyl ether, washed with $50 \mathrm{~mL}$ each of saturated aqueous sodium bicarbonate and brine, and dried over sodium sulfate. After filtration, the solvent was removed in vacuo to provide $(2 S, 3 R, 5 S)$ 1-allyl 2-ethyl 5-(2-bromophenyl)-3-(tert-butyl)-4-oxopyrrolidine-1,2-dicarboxylate (1.067 g, 2.359 mmol, $100 \%$ yield). ${ }^{1} \mathrm{H}$ NMR (400 MHz, DMSO- $\left.d_{6}\right) \delta \mathrm{ppm} 7.81(\mathrm{dd}, \mathrm{J}=7.8,1.7 \mathrm{~Hz}, 1 \mathrm{H}), 7.59(\mathrm{dd}, \mathrm{J}=$ 8.0, 1.3 Hz, 1H), 7.33 (td, J = 7.6, 1.3 Hz, 1H), 7.19 (ddd, J = 8.0, 7.3, $1.7 \mathrm{~Hz}, 1 \mathrm{H}), 5.71$ (ddt, J = 16.9, 11.0, $5.2 \mathrm{~Hz}, 1 \mathrm{H}), 5.45(\mathrm{~s}, 1 \mathrm{H}), 5.10$ - $4.99(\mathrm{~m}, 2 \mathrm{H}), 4.64(\mathrm{dd}, \mathrm{J}=4.6,0.6 \mathrm{~Hz}, 1 \mathrm{H}), 4.46$ (dtd, J = 5.2, 1.5, $0.7 \mathrm{~Hz}, 2 \mathrm{H}), 4.29$ - $4.16(\mathrm{~m}, 2 \mathrm{H}), 2.63(\mathrm{dd}, \mathrm{J}=4.6,0.9 \mathrm{~Hz}, 1 \mathrm{H}), 1.30$ - $1.22(\mathrm{~m}, 3 \mathrm{H}), 1.06(\mathrm{~s}, 9 \mathrm{H})$; MS $(\mathrm{APCI}+) m / z 452(\mathrm{M}+\mathrm{H})^{+}$.

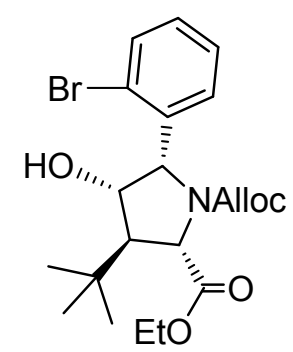

(2S,3R,4S,5S)-1-allyl 2-ethyl 5-(2-bromophenyl)-3-(tert-butyl)-4-hydroxypyrrolidine-1,2dicarboxylate (80)

Compound 79 (850 mg, $1.879 \mathrm{mmol})$ was dissolved in ethanol $(10 \mathrm{~mL})$ and cooled in an ice/acetone bath. Sodium tetrahydroborate $(85 \mathrm{mg}, 2.255 \mathrm{mmol}$ ) was added in small portions over 30 minutes. The 
reaction mixture was concentrated in vacuo (no heat) and $10 \mathrm{~mL}$ of saturated aqueous sodium bicarbonate and $15 \mathrm{~mL}$ of ethyl acetate were added. The reaction mixture was stirred at room temperature for 30 minutes. The organics were diluted with $50 \mathrm{~mL}$ more ethyl acetate, separated, washed with brine, dried over sodium sulfate, filtered, and concentrated. The crude residue was loaded onto a $24 \mathrm{~g}$ silica gel column and eluted with $5-100 \%$ ethyl acetate/heptanes to provide a $0.584 \mathrm{~g}$ of a mixture of diastereomers. The crude material was rechromatographed using a $24 \mathrm{~g}$ silica gel cartridge with a gradient of 5-30\% ethyl acetate/heptanes over 20 minutes to provide (2S,3R,4S,5S)-1-allyl 2-ethyl 5-(2-bromophenyl)-3(tert-butyl)-4-hydroxypyrrolidine-1,2-dicarboxylate (0.465 g, $1.023 \mathrm{mmol}, 54.5 \%$ yield). ${ }^{1} \mathrm{H}$ NMR (400 $\left.\mathrm{MHz}, \mathrm{DMSO}-d_{6}\right) \delta$ ppm $7.93(\mathrm{dd}, \mathrm{J}=7.9,1.7 \mathrm{~Hz}, 1 \mathrm{H}), 7.47$ (dd, J = 8.0, 1.2 Hz, 1H), 7.25 (t, J = 7.6 Hz, 1H), 7.09 (td, J = 7.7, 1.7 Hz, 1H), 5.69 (ddt, J = 17.7, 10.1, 5.0 Hz, 1H), 5.20 (d, J = 6.0 Hz, 1H), 5.05 $4.94(\mathrm{~m}, 2 \mathrm{H}), 4.51-4.42(\mathrm{~m}, 2 \mathrm{H}), 4.41-4.33(\mathrm{~m}, 3 \mathrm{H}), 4.20(\mathrm{~d}, \mathrm{~J}=7.0 \mathrm{~Hz}, 2 \mathrm{H}), 2.29$ (t, J = 2.8 Hz, 1H), $1.25(\mathrm{t}, \mathrm{J}=7.1 \mathrm{~Hz}, 3 \mathrm{H}), 1.00(\mathrm{~s}, 9 \mathrm{H})$; MS (APCI+) $m / z 454(\mathrm{M}+\mathrm{H})^{+}$.

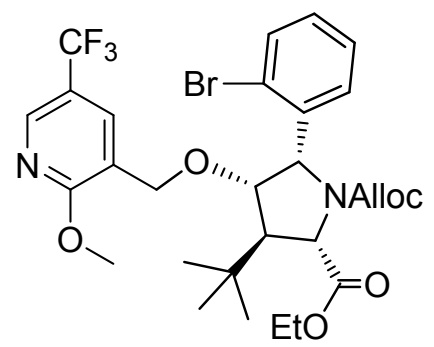

\section{1-allyl 2-ethyl (2S,3R,4S,5S)-5-(2-bromophenyl)-3-(tert-butyl)-4-((2-methoxy-5-} (trifluoromethyl)pyridin-3-yl)methoxy)pyrrolidine-1,2-dicarboxylate (81)

Compound 80 (6.92 g, $15.23 \mathrm{mmol}$ ) and 3-(bromomethyl)-2-methoxy-5-(trifluoromethyl)pyridine (5.16 $\mathrm{g}, 19.11 \mathrm{mmol})$ were dissolved in dimethylformamide $(61 \mathrm{~mL})$. The reaction was cooled to $0{ }^{\circ} \mathrm{C}$, and potassium tert-butoxide (1M in tetrahydrofuran, $19.0 \mathrm{~mL}, 19.0 \mathrm{mmol}$ ) was added dropwise. The reaction was stirred at ambient temperature for 1 hour. The reaction was poured into saturated aqueous $\mathrm{NH}_{4} \mathrm{Cl}(50$ $\mathrm{mL})$, diluted with water $(100 \mathrm{~mL})$ and extracted with methyl tert-butyl ether $(3 \times 100 \mathrm{~mL})$. The combined organics were washed with water $(100 \mathrm{~mL})$ and brine $(100 \mathrm{~mL})$, dried over $\mathrm{Na}_{2} \mathrm{SO}_{4}$, filtered and concentrated. The residue was purified by silica gel chromatography ( $0 \%$ to $5 \%$ ethyl acetate in dichloromethane) to provide the title compound (9.80 mg, 52\%). ${ }^{1} \mathrm{H}$ NMR (400 MHz, DMSO- $d_{6}, 120$ $\left.{ }^{\circ} \mathrm{C}\right) \delta \mathrm{ppm} 8.34-8.25(\mathrm{~m}, 1 \mathrm{H}), 7.96(\mathrm{dd}, \mathrm{J}=7.8,1.8 \mathrm{~Hz}, 1 \mathrm{H}), 7.45(\mathrm{dd}, \mathrm{J}=8.0,1.3 \mathrm{~Hz}, 1 \mathrm{H}), 7.22(\mathrm{td}, \mathrm{J}=$ 7.6, 1.3 Hz, 1H), 7.15 (d, J = 2.4 Hz, 1H), 7.08 (td, J = 7.6, $1.8 \mathrm{~Hz}, 1 \mathrm{H}), 5.72$ (ddt, J = 17.5, 10.0, 5.0 Hz, $1 \mathrm{H}), 5.33(\mathrm{~d}, \mathrm{~J}=5.6 \mathrm{~Hz}, 1 \mathrm{H}), 5.09$ - $4.99(\mathrm{~m}, 2 \mathrm{H}), 4.54(\mathrm{~d}, \mathrm{~J}=2.0 \mathrm{~Hz}, 1 \mathrm{H}), 4.47$ - 4.37 (m, 4H), 4.34 (dd, $\mathrm{J}=13.6,0.9 \mathrm{~Hz}, 1 \mathrm{H}), 4.12(\mathrm{qd}, \mathrm{J}=7.1,1.2 \mathrm{~Hz}, 2 \mathrm{H}), 3.88(\mathrm{~s}, 3 \mathrm{H}), 2.52(\mathrm{t}, \mathrm{J}=1.5 \mathrm{~Hz}, 1 \mathrm{H}), 1.15(\mathrm{t}, \mathrm{J}=7.1$ $\mathrm{Hz}, 3 \mathrm{H}), 1.05(\mathrm{~s}, 9 \mathrm{H})$; MS (ESI+) $m / z 643 \& 645(\mathrm{M}+\mathrm{H})^{+}$. 


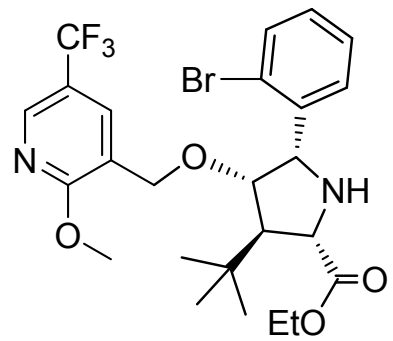

ethyl (2S,3R,4S,5S)-5-(2-bromophenyl)-3-(tert-butyl)-4-((2-methoxy-5-(trifluoromethyl)pyridin-3yl)methoxy)pyrrolidine-2-carboxylate (82)

Compound 81 (4.925 mg, $7.65 \mathrm{mmol})$ was dissolved in ethyl acetate $(15 \mathrm{~mL})$ and dichloromethane (15 $\mathrm{mL})$ and treated with 1,3-dimethylpyrimidine-2,4,6(1H,3H,5H)-trione $(2.39 \mathrm{~g}, 15.31 \mathrm{mmol})$ and tetrakis(triphenylphosphine)palladium $(94.5 \mathrm{mg}, 0.082 \mathrm{mmol})$. The reaction mixture was stirred at ambient temperature. After 1 hour, the reaction mixture was diluted with methyl tert-butyl ether (100 $\mathrm{mL})$ and stirred with $10 \%$ aqueous $\mathrm{Na}_{2} \mathrm{CO}_{3}(100 \mathrm{~mL})$ solution for 30 minutes. The phases were then separated, and the organic layer was washed with brine, dried over $\mathrm{Na}_{2} \mathrm{SO}_{4}$, filtered, and concentrated to provide the title compound $(4.28 \mathrm{~g}, 100 \%)$. ${ }^{1} \mathrm{H}$ NMR $\left(400 \mathrm{MHz}, \mathrm{DMSO}-d_{6}, 120{ }^{\circ} \mathrm{C}\right) \delta \mathrm{ppm} 8.35(\mathrm{dd}, \mathrm{J}=$ 2.5, 1.2 Hz, 1H), 7.75 (dd, J = 7.8, 1.8 Hz, 1H), 7.48 (dd, J = 7.9, $1.2 \mathrm{~Hz}, 1 \mathrm{H}), 7.26$ (td, J = 7.5, $1.3 \mathrm{~Hz}$, 1H), $7.15(\mathrm{~d}, \mathrm{~J}=2.5 \mathrm{~Hz}, 1 \mathrm{H}), 7.12(\mathrm{td}, \mathrm{J}=7.6,1.8 \mathrm{~Hz}, 1 \mathrm{H}), 5.61-5.43(\mathrm{~m}, 1 \mathrm{H}), 5.07-4.92(\mathrm{~m}, 1 \mathrm{H}), 4.35$ $(\mathrm{t}, \mathrm{J}=5.5 \mathrm{~Hz}, 1 \mathrm{H}), 4.28(\mathrm{~d}, \mathrm{~J}=14.1 \mathrm{~Hz}, 1 \mathrm{H}), 4.18-4.04(\mathrm{~m}, 2 \mathrm{H}), 3.89-3.78(\mathrm{~m}, 4 \mathrm{H}), 3.65(\mathrm{t}, \mathrm{J}=6.2 \mathrm{~Hz}$, $1 \mathrm{H}), 1.16(\mathrm{t}, \mathrm{J}=7.1 \mathrm{~Hz}, 3 \mathrm{H}), 0.95(\mathrm{~s}, 9 \mathrm{H}) ; \mathrm{MS}(\mathrm{ESI}+) \mathrm{m} / z 559 \& 561(\mathrm{M}+\mathrm{H})^{+}$.

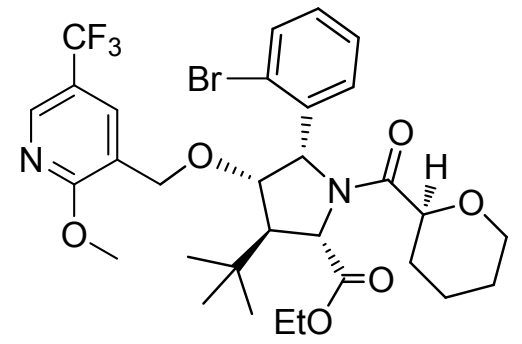

ethyl (2S,3R,4S,5S)-5-(2-bromophenyl)-3-(tert-butyl)-4-((2-methoxy-5-(trifluoromethyl)pyridin-3yl)methoxy)-1-((S)-tetrahydro-2H-pyran-2-carbonyl)pyrrolidine-2-carboxylate (83)

(S)-Tetrahydro-2H-pyran-2-carboxylic acid (1.21 g, $9.30 \mathrm{mmol})$ was dissolved in dichloromethane (40 $\mathrm{mL}$ ). Oxalyl chloride (2M in dichloromethane, $4.65 \mathrm{~mL}, 9.30 \mathrm{mmol}$ ) was added followed by dimethylformamide $(50 \mu \mathrm{L})$. The reaction was stirred at ambient temperature for 3 hours. The reaction was chilled to $0{ }^{\circ} \mathrm{C}$ and Compound $82(4.28 \mathrm{~g}, 7.65 \mathrm{mmol})$ and triethylamine $(4 \mathrm{~mL}, 28.7 \mathrm{mmol})$ dissolved in dichloromethane $(20 \mathrm{~mL})$ were added. The reaction was allowed to warm to ambient temperature and was stirred at ambient temperature for 17 hours. The mixture was diluted with 
dichloromethane $(100 \mathrm{~mL})$ and washed twice with saturated aqueous $\mathrm{NaHCO}_{3}$ and once with brine. The organic layer was dried over $\mathrm{Na}_{2} \mathrm{SO}_{4}$, filtered, and concentrated. The residue was purified by silica gel chromatography, eluting with $5 \%$ ethyl acetate in dichloromethane to provide the title compound (3.33 g, 65\%). ${ }^{1} \mathrm{H}$ NMR $\left(400 \mathrm{MHz}, \mathrm{DMSO}-d_{6}, 120{ }^{\circ} \mathrm{C}\right) \delta \mathrm{ppm} 8.29(\mathrm{~d}, \mathrm{~J}=1.7 \mathrm{~Hz}, 1 \mathrm{H}), 8.05(\mathrm{~d}, \mathrm{~J}=7.9 \mathrm{~Hz}, 1 \mathrm{H})$, $7.49(\mathrm{~d}, \mathrm{~J}=7.9 \mathrm{~Hz}, 1 \mathrm{H}), 7.24(\mathrm{~s}, 1 \mathrm{H}), 7.17(\mathrm{~d}, \mathrm{~J}=2.4 \mathrm{~Hz}, 1 \mathrm{H}), 7.11(\mathrm{~s}, 1 \mathrm{H}), 5.77(\mathrm{~s}, 1 \mathrm{H}), 4.73(\mathrm{~s}, 1 \mathrm{H})$, $4.38(\mathrm{~d}, \mathrm{~J}=6.0 \mathrm{~Hz}, 1 \mathrm{H}), 4.32(\mathrm{~d}, \mathrm{~J}=13.7 \mathrm{~Hz}, 1 \mathrm{H}), 4.09(\mathrm{qd}, \mathrm{J}=7.1,2.2 \mathrm{~Hz}, 2 \mathrm{H}), 3.98-3.90$ (m, 1H), $3.87(\mathrm{~s}, 3 \mathrm{H}), 3.83-3.73(\mathrm{~m}, 1 \mathrm{H}), 3.51-3.20(\mathrm{~m}, 2 \mathrm{H}), 2.45-2.38(\mathrm{~m}, 1 \mathrm{H}), 1.68(\mathrm{~s}, 1 \mathrm{H}), 1.45(\mathrm{~d}, \mathrm{~J}=54.8$ $\mathrm{Hz}, 5 \mathrm{H}), 1.14(\mathrm{t}, \mathrm{J}=7.1 \mathrm{~Hz}, 3 \mathrm{H}), 1.02(\mathrm{~s}, 9 \mathrm{H})$; MS (ESI+) $m / z 671 \& 673(\mathrm{M}+\mathrm{H})^{+}$.

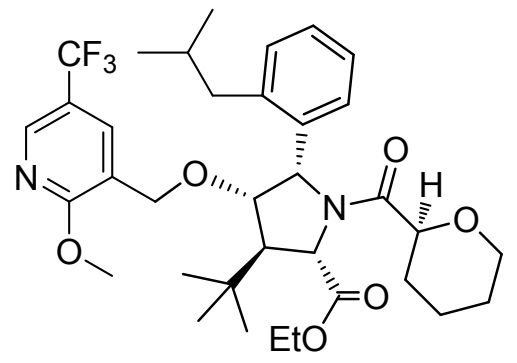

(2S,3R,4S,5S)-ethyl 3-(tert-butyl)-5-(2-isobutylphenyl)-4-((2-methoxy-5-(trifluoromethyl)pyridin-3yl)methoxy)-1-((S)-tetrahydro-2H-pyran-2-carbonyl)pyrrolidine-2-carboxylate (84)

Compound 83 (178C, $174 \mathrm{mg}, 0.259 \mathrm{mmol})$, isobutylboronic acid (52.8 mg, $0.518 \mathrm{mmol})$ and potassium carbonate $(107 \mathrm{mg}, 0.777 \mathrm{mmol})$ were suspended in toluene $(2.5 \mathrm{~mL})$. The reaction was purged with nitrogen for 2 minutes, and [1,1'-bis(diphenylphosphino)ferrocene]dichloropalladium(II) (10.58 mg, $0.013 \mathrm{mmol}$ ) was added. The reaction was capped and heated at $110^{\circ} \mathrm{C}$ for 4 hours. The solvent was reduced under a stream of nitrogen and the crude material was purified using a $10 \mathrm{~g}$ silica gel cartridge with a gradient of $5-100 \%$ ethyl acetate/heptanes over 20 minutes to give the title compound. ${ }^{1} \mathrm{H}$ NMR (400 MHz, DMSO-d6) $\delta$ ppm $8.28(\mathrm{~s}, 1 \mathrm{H}), 8.00(\mathrm{dd}, \mathrm{J}=7.7,1.4 \mathrm{~Hz}, 1 \mathrm{H}), 7.22(\mathrm{~s}, 1 \mathrm{H}), 7.13$ - 6.97 (m, $3 \mathrm{H}), 5.48(\mathrm{~s}, 1 \mathrm{H}), 4.71(\mathrm{~s}, 1 \mathrm{H}), 4.40-4.28(\mathrm{~m}, 1 \mathrm{H}), 4.25(\mathrm{~d}, \mathrm{~J}=13.8 \mathrm{~Hz}, 1 \mathrm{H}), 4.21(\mathrm{~d}, \mathrm{~J}=5.6 \mathrm{~Hz}, 1 \mathrm{H})$, $4.08(\mathrm{qd}, \mathrm{J}=7.1,3.3 \mathrm{~Hz}, 2 \mathrm{H}), 3.94(\mathrm{~d}, \mathrm{~J}=13.6 \mathrm{~Hz}, 1 \mathrm{H}), 3.87$ (s, 1H), 3.84 (s, 3H), 3.78 (d, J = 13.8 Hz, $1 \mathrm{H}), 3.73(\mathrm{~d}, \mathrm{~J}=12.9 \mathrm{~Hz}, 2 \mathrm{H}), 2.55(\mathrm{~d}, \mathrm{~J}=7.0 \mathrm{~Hz}, 1 \mathrm{H}), 2.43(\mathrm{~d}, \mathrm{~J}=1.8 \mathrm{~Hz}, 1 \mathrm{H}), 1.94(\mathrm{td}, \mathrm{J}=15.7,14.3$, $5.8 \mathrm{~Hz}, 1 \mathrm{H}), 1.66(\mathrm{~s}, 1 \mathrm{H}), 1.52(\mathrm{~d}, \mathrm{~J}=12.1 \mathrm{~Hz}, 1 \mathrm{H}), 1.36(\mathrm{~s}, 3 \mathrm{H}), 1.14(\mathrm{t}, \mathrm{J}=7.1 \mathrm{~Hz}, 3 \mathrm{H}), 1.02(\mathrm{~d}, \mathrm{~J}=2.0$ $\mathrm{Hz}, 9 \mathrm{H}), 0.93(\mathrm{t}, \mathrm{J}=6.4 \mathrm{~Hz}, 6 \mathrm{H})$; MS (APCI+) $m / z 649(\mathrm{M}+\mathrm{H})^{+}$. 


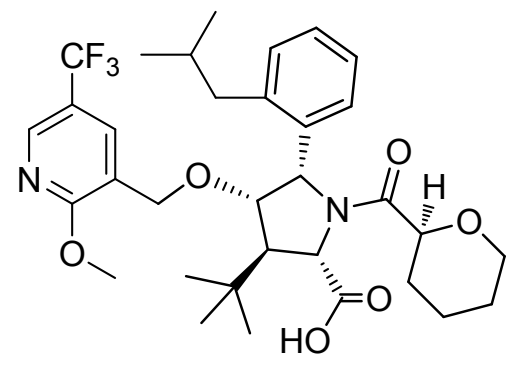

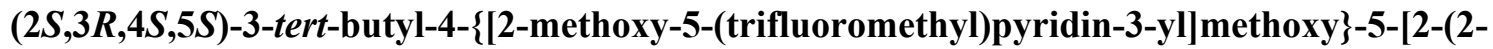
methylpropyl)phenyl]-1-[(2S)-oxane-2-carbonyl]pyrrolidine-2-carboxylic acid (18)

Compound $84(160 \mathrm{mg}, 0.247 \mathrm{mmol})$ was dissolved in methanol $(822 \mu \mathrm{l})$ and tetrahydrofuran $(822 \mu 1)$. A solution of lithium hydroxide $(56 \mathrm{mg}, 2.338 \mathrm{mmol})$ in water $(822 \mu \mathrm{l})$ was added. The reaction was heated at $45^{\circ} \mathrm{C}$ for 16 hours. The solvent was reduced in volume, and acidified with $1 \mathrm{M}$ aqueous $\mathrm{HCl}$ to $\mathrm{pH} \sim 3$. The reaction was extracted with dichloromethane. The solvent was removed and the crude material was purified by reverse-phase preparative HPLC on a Phenomenex ${ }^{\circledR}$ Luna ${ }^{\circledR}$ C8(2) $5 \mu \mathrm{m} 100 \AA$ AXIA $^{\mathrm{TM}}$ column $(30 \mathrm{~mm} \times 150 \mathrm{~mm})$. A gradient of acetonitrile $(\mathrm{A})$ and $0.1 \%$ trifluoroacetic acid in water (B) was used, at a flow rate of $50 \mathrm{~mL} /$ minute $(0-0.5$ minutes $10 \% \mathrm{~A}, 0.5-7.0$ minutes linear gradient $10-95 \% \mathrm{~A}$, 7.0-10.0 minutes $95 \%$ A, 10.0-12.0 minutes linear gradient $95-10 \% \mathrm{~A}$ ) to give the title compound. ${ }^{1} \mathrm{H}$ NMR (400 MHz, DMSO- $\left.d_{6}\right) \delta$ ppm 8.27 (dq, J = 2.0, $\left.1.0 \mathrm{~Hz}, 1 \mathrm{H}\right), 8.06(\mathrm{dd}, \mathrm{J}=7.6,1.5 \mathrm{~Hz}, 1 \mathrm{H}), 7.20$ (d, $\mathrm{J}=2.4 \mathrm{~Hz}, 1 \mathrm{H}), 7.06(\mathrm{q}, \mathrm{J}=8.8,7.1 \mathrm{~Hz}, 3 \mathrm{H}), 5.50(\mathrm{~s}, 1 \mathrm{H}), 4.67(\mathrm{~d}, \mathrm{~J}=1.7 \mathrm{~Hz}, 1 \mathrm{H}), 4.29(\mathrm{~d}, \mathrm{~J}=13.9 \mathrm{~Hz}$, $1 \mathrm{H}), 4.21(\mathrm{~d}, \mathrm{~J}=5.7 \mathrm{~Hz}, 1 \mathrm{H}), 4.07-3.97(\mathrm{~m}, 1 \mathrm{H}), 3.86(\mathrm{~s}, 3 \mathrm{H}), 3.84-3.79(\mathrm{~m}, 1 \mathrm{H}), 3.78$ - $3.70(\mathrm{~m}, 1 \mathrm{H})$, $3.18(\mathrm{~d}, \mathrm{~J}=6.3 \mathrm{~Hz}, 1 \mathrm{H}), 2.53(\mathrm{dd}, \mathrm{J}=7.1,4.0 \mathrm{~Hz}, 2 \mathrm{H}), 2.49(\mathrm{~d}, \mathrm{~J}=1.7 \mathrm{~Hz}, 1 \mathrm{H}), 1.91(\mathrm{dd}, \mathrm{J}=13.3,6.8$ Hz, 1H), 1.69 - 1.61 (m, 1H), 1.54 (q, J = 11.5 Hz, 1H), 1.35 (d, J = 11.8 Hz, 3H), 1.03 (s, 9H), 1.00 (d, J $=3.3 \mathrm{~Hz}, 1 \mathrm{H}), 0.92(\mathrm{t}, \mathrm{J}=6.8 \mathrm{~Hz}, 6 \mathrm{H})$; MS (ESI+) $m / z 621.3(\mathrm{M}+\mathrm{H})^{+}$.

Table 1. LRMS Table for compounds 5-19 and HPLC purity

\begin{tabular}{|c|c|c|c|c|}
\hline Compound & $\begin{array}{c}\text { Molecular } \\
\text { Formula }\end{array}$ & Mass Found & $\begin{array}{c}\text { Mass } \\
\text { Required }\end{array}$ & $\begin{array}{c}\text { HPLC } \\
\text { Purity }\end{array}$ \\
\hline $\mathbf{5}$ & $\mathrm{C}_{31} \mathrm{H}_{38} \mathrm{~F}_{3} \mathrm{NO}_{5}$ & MS (ESI-) $m / z 560.3(\mathrm{M}-\mathrm{H})^{-}$ & $560.3(\mathrm{M}-\mathrm{H})^{-}$ & $97.0 \%$ \\
\hline $\mathbf{6}$ & $\mathrm{C}_{31} \mathrm{H}_{38} \mathrm{~F}_{3} \mathrm{NO}_{5}$ & MS (ESI+) $m / z 562.3(\mathrm{M}+\mathrm{H})^{+}$ & $562.3(\mathrm{M}+\mathrm{H})^{+}$ & $96.7 \%$ \\
\hline $\mathbf{7}$ & $\mathrm{C}_{31} \mathrm{H}_{38} \mathrm{~F}_{3} \mathrm{NO}_{5}$ & MS (ESI+) $m / z 562.3(\mathrm{M}+\mathrm{H})^{+}$ & $562.3(\mathrm{M}+\mathrm{H})^{+}$ & $97.1 \%$ \\
\hline $\mathbf{8}$ & $\mathrm{C}_{30} \mathrm{H}_{37} \mathrm{~F}_{3} \mathrm{~N}_{2} \mathrm{O}_{5}$ & MS (ESI-) $m / z 561.3(\mathrm{M}-\mathrm{H})^{-}$ & $561.3(\mathrm{M}-\mathrm{H})^{-}$ & $98.1 \%$ \\
\hline $\mathbf{9}$ & $\mathrm{C}_{34} \mathrm{H}_{46} \mathrm{~N}_{2} \mathrm{O}_{5}$ & MS (APCI+) $m / z 563.1(\mathrm{M}+\mathrm{H})^{+}$ & $563.3(\mathrm{M}+\mathrm{H})^{+}$ & $97.3 \%$ \\
\hline $\mathbf{1 0}$ & $\mathrm{C}_{33} \mathrm{H}_{46} \mathrm{~N}_{2} \mathrm{O}_{5}$ & MS (ESI-) $m / z 549.3(\mathrm{M}-\mathrm{H})^{-}$ & $549.3(\mathrm{M}+\mathrm{H})^{+}$ & $96.9 \%$ \\
\hline $\mathbf{1 1}$ & $\mathrm{C}_{27} \mathrm{H}_{33} \mathrm{~F}_{3} \mathrm{~N}_{2} \mathrm{O}_{6}$ & MS (APCI+) $m / z 539.2(\mathrm{M}+\mathrm{H})^{+}$ & $539.2(\mathrm{M}+\mathrm{H})^{+}$ & $98.2 \%$ \\
\hline $\mathbf{1 2}$ & $\mathrm{C}_{29} \mathrm{H}_{35} \mathrm{~F}_{3} \mathrm{~N}_{2} \mathrm{O}_{6}$ & MS (ESI+) $m / z 565.1(\mathrm{M}+\mathrm{H})^{+}$ & $565.2(\mathrm{M}+\mathrm{H})^{+}$ & $97.3 \%$ \\
\hline $\mathbf{1 3}$ & $\mathrm{C}_{29} \mathrm{H}_{35} \mathrm{~F}_{3} \mathrm{~N}_{2} \mathrm{O}_{6}$ & MS (ESI+) $m / z 565.1(\mathrm{M}+\mathrm{H})^{+}$ & $565.2(\mathrm{M}+\mathrm{H})^{+}$ & $98.5 \%$ \\
\hline $\mathbf{1 4}$ & $\mathrm{C}_{32} \mathrm{H}_{41} \mathrm{~F}_{3} \mathrm{~N}_{2} \mathrm{O}_{6}$ & MS (ESI+) $m / z 605.3(\mathrm{M}+\mathrm{H})^{-}$ & $605.3(\mathrm{M}+\mathrm{H})^{-}$ & $97.9 \%$ \\
\hline $\mathbf{1 5}$ & $\mathrm{C}_{32} \mathrm{H}_{39} \mathrm{~F}_{3} \mathrm{~N}_{2} \mathrm{O}_{6}$ & MS (ESI+) $m / z 605.1(\mathrm{M}+\mathrm{H})^{+}$ & $605.2(\mathrm{M}+\mathrm{H})^{+}$ & $98.4 \%$ \\
\hline
\end{tabular}




\begin{tabular}{|c|c|c|c|c|}
\hline $\mathbf{1 6}$ & $\mathrm{C}_{29} \mathrm{H}_{34} \mathrm{ClF}_{3} \mathrm{~N}_{2} \mathrm{O}_{6}$ & MS (ESI+) $m / z 599.2(\mathrm{M}+\mathrm{H})^{+}$ & $599.2(\mathrm{M}+\mathrm{H})^{+}$ & $96.3 \%$ \\
\hline $\mathbf{1 7}$ & $\mathrm{C}_{31} \mathrm{H}_{39} \mathrm{~F}_{3} \mathrm{~N}_{2} \mathrm{O}_{6}$ & MS (APCI+) $m / z 593.4(\mathrm{M}+\mathrm{H})^{+}$ & $593.3(\mathrm{M}+\mathrm{H})^{+}$ & $97.6 \%$ \\
\hline $\mathbf{1 8}$ & $\mathrm{C}_{33} \mathrm{H}_{43} \mathrm{~F}_{3} \mathrm{~N}_{2} \mathrm{O}_{6}$ & MS (ESI+) $m / z 621.3(\mathrm{M}+\mathrm{H})^{+}$ & $621.3(\mathrm{M}+\mathrm{H})^{+}$ & $97.0 \%$ \\
\hline $\mathbf{1 9}$ & $\mathrm{C}_{30} \mathrm{H}_{37} \mathrm{~F}_{3} \mathrm{~N}_{2} \mathrm{O}_{6}$ & MS (ESI+) $m / z 579.3(\mathrm{M}+\mathrm{H})^{+}$ & $579.3(\mathrm{M}+\mathrm{H})^{+}$ & $98.7 \%$ \\
\hline
\end{tabular}

\section{Biology}

Determination of Biological Activity

\section{Cellular Assays}

\section{Cell Surface Expression-Horse Radish Peroxidase (CSE-HRP) Assay:}

A cellular assay for measuring the F508del CFTR cell surface expression after correction with test compounds either without or with a co-corrector $(2 \mu \mathrm{M}$ of $3-[(2 R, 4 R)-4-(\{[1-(2,2-$ difluoro-1,3-benzodioxol-5-yl)cyclopropyl]carbonyl \}amino)-7-methoxy-3,4-dihydro-2Hchromen-2-yl]benzoic acid, 1), was developed in human lung derived epithelial cell line (CFBE41o-). ${ }^{1}$ This was achieved by expressing the F508del CFTR mutation along with a horseradish peroxidase (HRP) in the fourth exofacial loop and then measuring the HRP activity using luminescence readout from these cells, CFBE41o-F508del CFTR-HRP, that were incubated overnight with the test corrector compounds. ${ }^{2}$ Briefly, for this primary assay, the CFBE41o-F508del CFTR-HRP cells were plated in 384-well plates at 4,000 cells/well along with $0.5 \mu \mathrm{g} / \mathrm{mL}$ doxycycline to induce the F508del CFTR-HRP expression and further incubated at $37{ }^{\circ} \mathrm{C}, 5 \% \mathrm{CO}_{2}$ for 72 hours. The test compounds were then added at the required concentrations and further incubated for $18-24$ hours at $33^{\circ} \mathrm{C}$. The highest concentration tested was $20 \mu \mathrm{M}$ with an 8-point concentration response curve using a 3-fold dilution. Three replicate plates were run to determine one $\mathrm{EC}_{50}$. All plates contained negative controls (dimethyl sulfoxide, DMSO) and positive controls (3 $\mu \mathrm{M}$ of 3-[(2R,4R)-4-(\{[1-(2,2-difluoro-1,3- 
benzodioxol-5-yl)cyclopropyl]carbonyl $\}$ amino)-7-methoxy-3,4-dihydro-2H-chromen-2-

yl]benzoic acid) (Compound $\mathbf{1}$ ) as well as on-plate concentration response of the positive control. Post incubation, the plates were washed $5 \times$ times with Dulbecco's phosphate buffered saline (DPBS), followed by the addition of the HRP substrate, luminol $(50 \mu \mathrm{L})$, and measuring the HRP activity using luminescence readout on EnVision ${ }^{\circledR}$ Multilabel Plate Reader. The raw counts from the experiment are analyzed using Accelrys ${ }^{\circledR}$ Assay Explorer v3.3.

The $\%$ activity measured at each of the 8 test concentrations of the test compound was normalized to the on-plate positive control using the following formula:

$\%$ activity $=[$ (test compound response - DMSO response) $/$ (positive control response - DMSO response) $]^{*} 100$

The maximum $\%$ activity achieved for the test compound at any tested concentration is presented in Tables along with the $\mathrm{EC}_{50}$ calculated using the general sigmoidal curve with variable Hill slope equation.

\section{Trans-epithelial Current Clamp on Human Bronchial Epithelial Cells Conductance}

\section{Assay:}

A cell based assay using the primary human bronchial epithelial cells (hBE) was used as a secondary assay to test novel F508del CFTR correctors for their activity on primary hBE cells with F508del/F508del CFTR mutation. Primary human bronchial epithelial (hBE) cells from F508del/F508del CFTR patients were expanded from $1 \times 10^{6}$ to $250 \times 10^{6}$ cells. ${ }^{3}$ For this purpose, cells isolated from CF patients with the homozygous mutation were seeded onto 24 well Corning (Cat \# 3378) filter plates that were coated with 3 T3 conditioned media and grown at an air-liquid interface for 35 days using an Ultroser ${ }^{\circledR} \mathrm{G}$ supplemented differentiation media. Apical surface mucus was removed 72 hours before the experiment by incubating the apical surface of the cells 
for 30 minutes with $3 \mathrm{mM}$ dithiothreitol (DTT) prepared in the differentiation media, followed by aspiration of the mucus along with the media. The apical surface is washed again with phosphate buffered saline (PBS) incubated for 30 minutes followed with aspiration. The cells were then incubated with the desired dose of the corrector compounds $18-24$ hours at $37{ }^{\circ} \mathrm{C}, 5 \%$ $\mathrm{CO}_{2}$. The corrector compounds were prepared as $10 \mathrm{mM}$ stocks and the desired concentrations were prepared in differentiation media and were always applied on the basolateral side of the epithelial cells.

On the day of measuring the corrector activity on the TECC, the cells were switched into a bicarbonate and serum free F-12 Coon's medium and allowed to equilibrate for 90 minutes in a $\mathrm{CO}_{2}$ free incubator. At the time of measurement, the apical and basolateral sides of the filter were bathed with the F-12 Coon's modification media (with $20 \mathrm{mM}$ 4-(2-hydroxyethyl)-1piperazineethanesulfonic acid (HEPES), pH 7.4 (using $1 \mathrm{M}$ tris(hydroxymethyl)aminomethane (Tris)), and the measurements were made at $36.5^{\circ} \mathrm{C}$. Current responses before and after the sequential addition of benzamil (apical $6 \mu \mathrm{M}$ addition; for inhibiting epithelial $\mathrm{ENaC}$ channel), forskolin (apical and basolateral $10 \mu \mathrm{M}$ addition; for activating the CFTR channel), control potentiator ( $N$-(3-carbamoyl-5,5,7,7-tetramethyl-4,7-dihydro-5H-thieno[2,3-c]pyran-2-yl)- $1 H$ pyrazole-5-carboxamide; apical and basolateral $0.75 \mu \mathrm{M}$ addition; for potentiating the CFTR channel) and bumetanide (basolateral $20 \mu \mathrm{M}$ addition; for inhibiting the $\mathrm{Na}: 2 \mathrm{Cl}: \mathrm{K}$ cotransporter, an indirect measure of inhibiting the Cl- secretion driven by CFTR channel) were measured. The assay uses a TECC-24 (Transepithelial Current Clamp for 24 wells) instrument that measures the functionality of the mutated channel by measuring the equivalent CFTR current $\left(\mathrm{I}_{E Q}\right)$ generated by the polarized primary epithelial cells. The instrument works by measuring the transepithelial potential difference $\left(\mathrm{V}_{T}\right)$ and transepithelial conductance $\left(\mathrm{G}_{T}\right)$ 
under current clamp conditions using a custom designed multi-channel current clamp and electrode manifold. Each measured $\mathrm{V}_{\mathrm{T}}$ values are corrected for the electrode offset potential, and each measured $\mathrm{G}_{\mathrm{T}}$ values are corrected for the combined solution series and empty filter resistances. The corrected $\mathrm{V}_{\mathrm{T}}$ and $\mathrm{G}_{\mathrm{T}}$ values were then used to calculate the equivalent current, $\mathrm{I}_{E Q}$ using Ohm's law $\left(I_{E Q}=\mathrm{V}_{\mathrm{T}} \cdot \mathrm{G}_{\mathrm{T}}\right)$. In addition to calculating the $I_{E Q}$, the area under the curve (AUC) for the time period between the forskolin peak $I_{E Q}$ response and at the time of bumetanide addition was also calculated using a one-third trapezoid method. The assay was run in a 24 -well format and all 24-wells were measured at the same time point giving a higher throughput for this assay.

All plates contained negative controls (dimethyl sulfoxide, DMSO) which coupled with the control potentiator $(0.75 \mu \mathrm{M}$ of $\mathrm{N}$-(3-carbamoyl-5,5,7,7-tetramethyl-4,7-dihydro-5Hthieno[2,3-c]pyran-2-yl)-1H-pyrazole-5-carboxamide, GLPG1837) sets the null response and positive controls $(0.15 \mu \mathrm{M}$ of 4-[(2R,4R)-4-[[1-(2,2-difluoro-1,3-benzodioxol-5yl)cyclopropanecarbonyl]amino]-7-(difluoromethoxy)chroman-2-yl]benzoic acid ABBV-2222) coupled with the control potentiator sets the $100 \%$ response to measure the correction of the mutated CFTR channel. The specificity of the Forskolin-activated current is via CFTR activation, as demonstrated by inhibition with CFTR Inh172. The maximum percent activity (Emax) is reported relative to the positive control value.

The $\%$ activity measured at each of the 6 test concentrations of the test compound was normalized to the on-plate positive control using the following formula:\% activity $=[$ (test compound response - DMSO response) / (positive control response - DMSO response)]*100 The $I_{E Q}$ and AUC at different test concentrations were fit and an $\mathrm{EC}_{50}$ was calculated using the general sigmoidal curve with variable Hill slope equation included in the Prism $v 5$ software. 


\section{CYP3A4 Induction:}

Cryopreserved primary human hepatocytes were thawed and cultured overnight prior to treatment. Cultured hepatocytes were treated with either test compounds $(10 \mu \mathrm{M})$, vehicle control $(0.1 \% \mathrm{v} / \mathrm{v}$ DMSO), or prototypical inducer of CYP3A4 (rifampin $10 \mu \mathrm{M})$ for 48 hours, with culture medium being refreshed every 24 hours. Following the 48 hour treatment, CYP3A4 mRNA levels measured in compound treated hepatocytes were expressed as a percentage of the response of positive control (Rifampin $10 \mu \mathrm{M}$ ). In test compound treated hepatocytes, CYP3A4 mRNA level increase by less than $20 \%$ of the response of positive control (Rifampin) is considered low risk for CYP3A4 induction.

\footnotetext{
${ }^{1}$ Ehrhardt, C.; Collnot, E. M.; Baldes, C.; Becker, U.; Laue, M.; Kim, K. J.; Lehr, C. M. Towards an in vitro model of cystic fibrosis small airway epithelium: characterisation of the human bronchial epithelial cell line CFBE41o. Cell Tissue Res. 2006, 323, 405-415.

${ }^{2}$ Veit, G.; Avramescu, R. G.; Perdomo, D.; Phuan, P. W.; Bagdany, M.; Apaja, P. M.; Borot, F.; Szollosi, D.; Wu, Y. S.; Finkbeiner, W. E.; Hegedus, T.; Verkman, A. S.; Lukacs, G. L. Some gating potentiators, including VX-770, diminish deltaF508-CFTR functional expression. Sci. Transl. Med. 2014, 6, 246-297.

${ }^{3}$ Neuberger, T.; Burton, B.; Clark, H.; Van Goor, F., Use of primary cultures of human bronchial epithelial cells isolated from cystic fibrosis patients for the pre-clinical testing of CFTR modulators. In Methods Mol Biol, 2011; Vol. 741, pp 39-54.
} 